\title{
MICRODEFECTS \\ IN DISLOCATION-FREE SILICON CRYSTALS
}

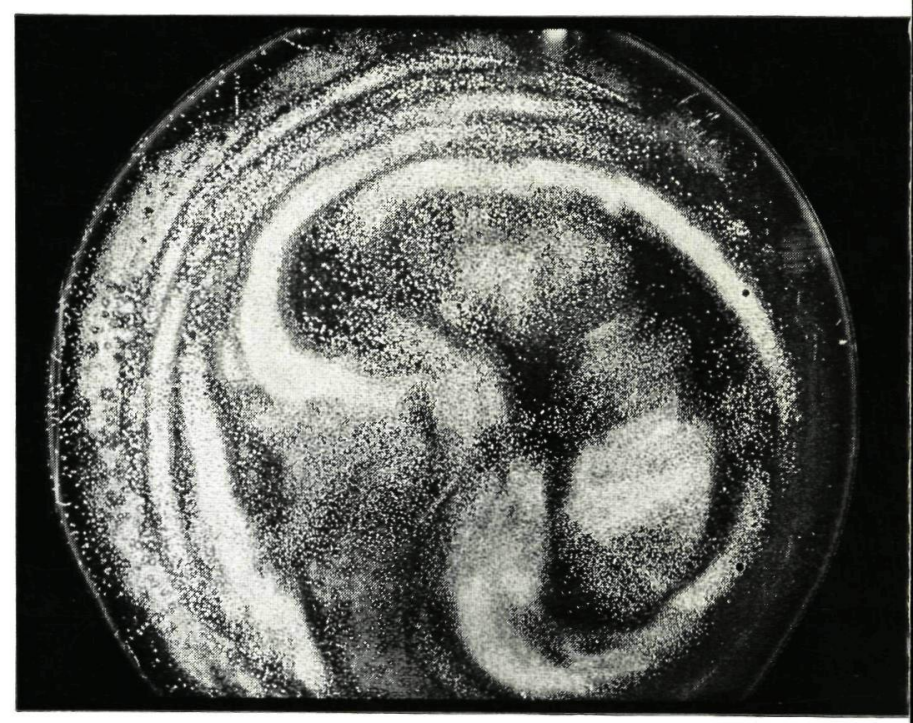


Cover picture:

Microdefects in dislocation-free Si. (this thesis, sec. 3.3). 


\section{MICRODEFECTS \\ IN DISLOCATION-FREE SILICON CRYSTALS}

\section{PROEFSCHRIFT}

TER VERKRIJGING VAN DE GRAAD VAN DOCTOR IN DE WISKUNDE EN NATUURWETENSCHAPPEN AAN DE KATHOLIEKE UNIVERSI IEIT TE NIJMEGEN, OP GEZAG VAN DE RECTOR MAGNIFICUS PROF. MR. F. J. F. M. DUYNSTEE VOLGENS BESLUIT VAN HET COLLEGE VAN DECANEN IN HET OPENBAAR TE VERDEDIGEN OP DONDERDAG 22 MAART 1973, DES NAMIDDAGS

TE 4.00 UUR

DOOR

ARIE JAN RUDOLF DE KOCK

GEBOREN TE AMERSFOORT 
PROMOTOR: PROF. DR. J. BLOEM

COPROMOTOR: PROF. DR. P. WYDER 
Aan Christine 


\section{Acknowledgement}

The investigations described in this thesis were performed at the Philips Research Laboratories, Waalre, Netherlands. I am greatly indebted to the management of these laboratories for affording me the opportunity to publish the results of these investigations in the present form.

I would like to express my gratitude to all those who have contributed to this work in any way, in particular to Mr. P. G. T. Boonen for his active assistance with the crystal characterization and Mr. P. J. Roksnoer for carrying out most of the crystal-growth experiments.

Finally I wish to thank Mr. J. Goorissen for many valuable discussions and Dr. L. J. Giling for his critical reading of the manuscript. 


\section{CONTENTS}

1. IMPERFECTIONS IN SILICON CRYSTALS ......... 1

1.1. Introduction and scope of present investigation . . . . . . . . 1

1.2. Silicon-crystal-growth methods . . . . . . . . . . . . . . . 1

1.2.1. The Czochralski method . . . . . . . . . . . . . . . 1

1.2.2. The floating-zone method . . . . . . . . . . . . 3

1.2.3. The growth of dislocation-free silicon crystals. . . . . . 4

1.3. Point defects in melt-grown silicon crystals . . . . . . . . . . 5

1.3.1. Impurities in silicon . . . . . . . . . . . . . 5

1.3.2. Impurity striations . . . . . . . . . . . . . . . . . 8

1.3.3. Vacancies and interstitials in silicon crystals . . . . . . 9

1.3.3.1. Equilibrium concentrations of vacancies and interstitials . . . . . . . . . . . . . . . . 9

1.3.3.2. The mobility of vacancies in silicon . . . . . . 10

1.3.3.3. Vacancy-impurity-complex formation . . . . 11

1.3.3.4. Vacancy clusters in dislocation-free silicon . . . 12

References . . . . . . . . . . . . . . . . . . . . 13

2. METHODS FOR DETECTING MICRODEFECTS IN SILICON CRYSTALS .................... . . . . 15

2.1. Introduction .. . . . . . . . . . . . . . . . 15

2.2. Preferential etching ... . . . . . . . . . . . . . 15

2.3. The copper-decoration technique . . . . . . . . . . 15

2.4. The lithium-decoration technique . . . . . . . . . . . . . 17

2.5. X-ray transmission topography . . . . . . . . . . . . . . . 19

References .. . . . . . . . . . . . . . . . . 23

3. EXPERIMENTAL RESULTS .. . . . . . . . . . . . . . 24

3.1. Introduction . . . . . . . . . . . . . . . . . . . . . . . 24

3.2. Results obtained with preferential etching . . . . . . . . . 24

3.3. Results obtained with copper decoration. . . . . . . . . . . 28

3.4. Comments on the results obtained with etching and copper decoration . . . . . . . . . . . . . . . . . . . . . . 42

3.5. Results obtained with lithium decoration ......... . 46

3.6. The influence of microdefects on the performance of micro-electronic devices . . . . . . . . . . . . . . . . . . . . . . 49

3.6.1. Introduction . . . . . . . . . . . . . . . . . . . . 49

3.6.2. Annealing experiments in nitrogen ... . . . . . 52

3.6.3. Oxidation . . . . . . . . . . . . . . . . . . . 54

3.6.4. Epitaxy ................. 54 
3.6.5. The influence of microdefects on the properties of planar diodes................ . . 57

References .................... 61

4. DISCUSSION . . . . . . . . . . . . . 62

4.1. The character of the grown-in microdefects 62

4.2. The shape of the vacancy clusters . 64

4.3. The size of the vacancy clusters . . . . . . . . . . . 65

4.4. Determination of the concentration of vacancy clusters . . . . 66

4.5. The nature of the vacancy-nucleation sites: homogene 3 is versus heterogeneous formation . . . . . . . . . 68

4.6. The model . . . . . . . . . . . . . . . . . . . . 70

4.7. Explanation of the experimental results . . . . 75

References .............. 81

5. THE ELIMINATION OF VACANCY-CLUSTER FORMATION DURING CRYSTAL GROWTH . . . . . . . . . . . . . . 83

5.1. Introduction . . . . . . . . . . . . . . . . . . . . . . 83

5.2. Methods for the elimination of vacancy clusters 83

5.2.1. Method I . . . . . . . . . . . . . . . . . . . . . 83

5.2.2. Method II . . . . . . . . . . . . . . . . . . . . . 84

5.2.3. Method III . . . . . . . . . . . . . . . . . . . . . 84

5.3. Experimental results and discussion . . . . . . . . . . . . . 86

5.3.1. Methods I and II . . . . . . . . . . . . . . . . . . 86

5.3.2. Method III . . . . . . . . . . . . . . . . . . . . . 86

5.3.3. Differences in the formation of $\mathrm{V}-\mathrm{O}$ associates at $500{ }^{\circ} \mathrm{C}$ in crystals with and without hydrogen dope 97

5.3.4. The incubation time for cluster formation . . . . . . . 101

5.3.5. The perfection of vacancy-cluster-free crystals . . . . . 101

References . . . . . . . . . . . . . . . . . 102

List of symbols . . . . . . . . . . . . . . . . . . . . . . 103

Summary .. . . . . . . . . . . . . . . . . . . . 106

Samenvatting . . . . . . . . . . . . . . . . . . . . . 107

Curriculum vitae . . . . . . . . . . . . . . . 109 


\section{IMPERFECTIONS IN SILICON CRYSTALS}

\subsection{Introduction and scope of present investigation}

In the field of semiconductor-device technology silicon single crystals are frequently used as the starting material. In particular the techniques now available for making planar devices and integrated circuits are almost exclusively based on silicon semiconductor crystals.

The electrical properties of micro-electronic devices, such as leakage current and breakdown voltage, depend to a great extent on the purity, homogeneity and perfection of the silicon crystals used. In the course of many years' research, crystal-growth techniques have therefore been improved to such an extent that silicon crystals containing neither dislocations nor impurity precipitates, can nowadays be grown from the melt.

However, particularly in the absence of the above-mentioned defects, certain types of microdefects may be formed in the crystal lattice.

During growth from the melt the crystal region adjacent to the melt will contain the equilibrium concentration of vacancies associated with the melting point of silicon $\left(1420^{\circ} \mathrm{C}\right)$. Because the equilibrium concentration of vacancies decreases with temperature (sec. 1.3.3) the as-grown material will become supersaturated with these point defects on cooling to room temperature. If the crystal contains dislocations, the excess vacancies can easily be annihilated at the jogs present along these line defects, resulting in climb of the dislocations. However, if the crystal is grown essentially free of dislocations, no vacancy sinks are present in the bulk. Consequently, as was first pointed out by Dash ${ }^{1-1}$ ), a large supersaturation of vacancies in the interior of the growing crystal has to be expected. This may lead to nucleation of vacancies, finally resulting in the formation of vacancy clusters. Evidence for the presence of such vacancy clusters in dislocation-free germanium crystals was found by Tweet ${ }^{1-2,3}$ ) and in dislocation-free silicon by Plaskett ${ }^{1-4}$ ) (sec. 1.3.3.4).

In this thesis a detailed study of microdefects, in particular vacancy clusters, in dislocation-free high-purity silicon crystals is presented. The concentration and distribution of the defects were determined using combinations of existing measuring techniques including $X$-ray transmission topography and copper decoration, supplemented by new techniques such as low-temperature lithium decoration.

\subsection{Silicon-crystal-growth methods}

\subsubsection{The Czochralski method}

One method which is often used for growing silicon single crystals is the Czochralski method (C.Z.) ${ }^{\mathbf{1}-5.6}$ ). This method is shown schematically in 


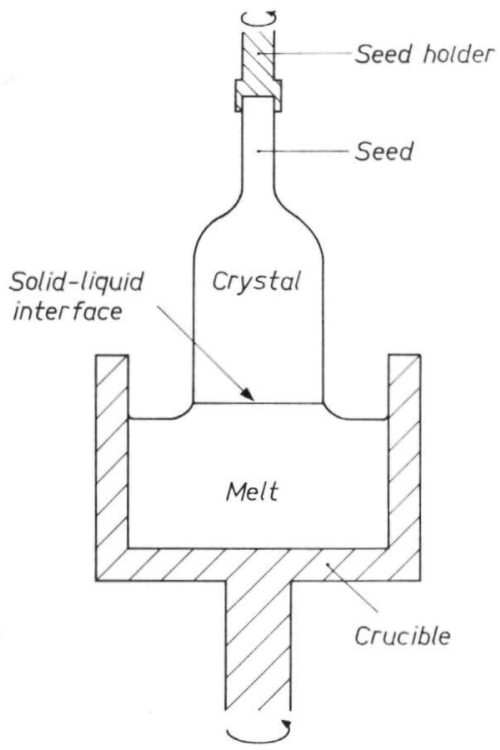

Fig. 1.1. The Czochralski crystal-growth method.

fig. 1.1. The first step is to melt polycrystalline silicon in a silica crucible by resistance heating or high-frequency heating. The required amount of dope is added to the melt. A thin seed crystal is then dipped into this melt. A small portion of the seed is intentionally melted to ensure that the melt wets the seed. The temperature is then lowered and the seed slowly withdrawn from the melt in the upward direction. This results in solidification of silicon at the solid-liquid interface in the same single-crystalline form and orientation as that of the seed. The crystal diameter can be increased by lowering the temperature of the melt. Crystals with large diameters $(>10 \mathrm{~cm})$ can be grown in this way. Both the crystal and the crucible are generally rotated in order to minimize thermal asymmetries. The crystal thus acquires the shape of a regular cylinder, while the stirring of the melt due to the rotation causes a more homogeneous dope distribution in the as-grown silicon.

This crystal-growth method, however, has one great disadvantage. Because molten silicon is chemically very reactive, the direct contact between the melt and the wall of the silica crucible causes contamination of the melt. This results in a relatively high oxygen concentration in the melt. Moreover, the crucible itself is usually heated by means of a carbon element in the case of resistance heating or by a carbon susceptor if high-frequency heating is used. This causes carbon contamination of the melt. Consequently, high amounts of oxygen and carbon are incorporated in the growing crystal. To eliminate this contamination crucible-less crystal-growth methods, known as floating-zone techniques ${ }^{1-7}$ ) (F.Z.), have been developed, 


\subsubsection{The floating-zone method}

Several versions of this technique exist. One of these, known as the pedestalpulling method ${ }^{1-8}$ ), was used in the present study. This method is sketched in fig. 1.2. The top of a vertical polycrystalline silicon rod (pedestal) is melted

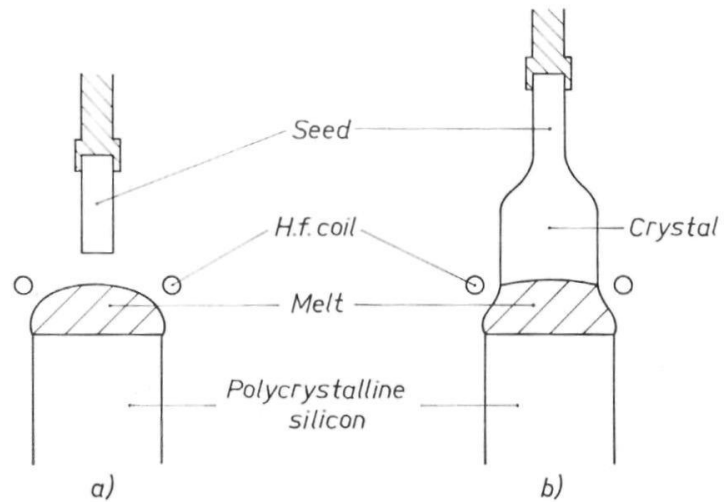

Fig. 1.2. The pedestal-pulling crystal-growth method.

with the aid of a water-cooled high-frequency induction coil. Low-resistivity silicon can be melted directly, while in the case of high-resistivity material the pedestal has to be pre-heated in another way before sufficiently strong eddy currents can be generated in the rod. Because of the low density of molten silicon $\left(2.42 \mathrm{~g} / \mathrm{cm}^{3}\right)$ and its high surface tension $(720 \mathrm{dyn} / \mathrm{cm})$, this melt is easily kept in position. A seed is dipped into the melt from above. A single crystal is subsequently grown by slow withdrawal of the seed in a manner similar to that adopted to the C.Z. method (fig. 1.2(b)). The diameter of the growing crystal can be increased by decreasing the energy of the applied electromagnetic field and therefore the temperature near the solid-liquid interface, but the maximum diameter will always be smaller than that of the polycrystalline rod used. To keep the melt stationary in relation to the h.f. coil the pedestal is also moved upward. The speed of the pedestal is determined by the crystalgrowth rate and the difference in diameter between the crystal and the pedestal. Both the seed and the pedestal are rotated around their vertical axes in order to reduce thermal asymmetries in the system. This growth process can be carried out in a water-cooled metal bell-jar or in a quartz tube. In the latter case the high-frequency coil is usually located outside the tube. Pedestal-pulled crystals are generally grown in vacuum or in an inert-gas atmosphere (purified Ar or $\mathrm{He})$.

In this crucible-less crystal-growth process the melt is in direct contact with silicon only. Consequently, severe contamination by unwanted impurities such as oxygen and carbon can be avoided. The final impurity content will be deter- 
mined by the purity of the atmosphere used and that of the polycrystalline starting material.

\subsubsection{The growth of dislocation-free silicon crystals}

Silicon crystals grown by the C.Z. or F.Z. methods usually contain high dislocation densities. The line defects present in the seed and which emerge at the solid-liquid interface will propagate into the growing crystal because a dislocation can only terminate at another dislocation or at the external crystal surface. Even if a dislocation-free seed is used dislocations will be created by the thermal shock originating from dipping the seed into the melt. Furthermore, because of radial temperature gradients present in the crystal during growth, internal stresses are built up. This leads to the generation of new dislocations $\left.{ }^{1-9,10}\right)$ in the part of the crystal where the temperature is in the plastic region $\left(T>600^{\circ} \mathrm{C}\right)$. Several dislocation-generation mechanisms are known to be active in silicon, e.g. the Frank-Read mechanism ${ }^{1-11}$ ) as found by Dash $\left.{ }^{1-12}\right)$. The average dislocation densities are usually lower in C.Z. silicon $\left(10^{3}-10^{4} \mathrm{~cm}^{-2}\right)$ than in F.Z. silicon $\left(10^{5}-10^{6} \mathrm{~cm}^{-2}\right)$ because of the higher radial temperature gradients in the latter.

However, it has been shown ${ }^{1-13}$ ) that dislocations are not thermodynamically stable. Their energy of formation is large, whereas the configurational entropy of the crystal is not appreciably increased by the presence of dislocations. It should therefore be possible to grow crystals from the melt which are completely dislocation-free. Indeed, a method has been developed by Dash ${ }^{1-1,14}$ ) for growing dislocation-free silicon. This method, which can also be used for the growth of dislocation-free crystals of $\mathrm{Ge}^{1-15}$ ) and $\mathrm{GaAs}^{1-16}$ ), for example, is shown schematically in fig. 1.3. After the seed is dipped into

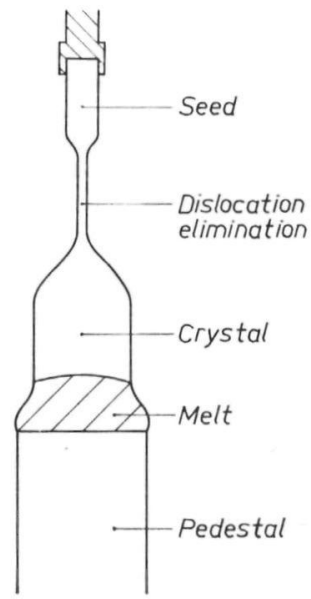

Fig. 1.3. The elimination of dislocations during crystal growth from the melt. 
the melt a thin neck is grown at a high growth rate of about $15 \mathrm{~mm} / \mathrm{min}$. The diameter of the neck is kept at about $2 \mathrm{~mm}$. The dislocation network, generally present in the seed after it is dipped into the melt, does not propagate into the thin neck. A reasonable explanation is the following ${ }^{1-17,18}$ ). In the thin neck which is grown at a high growth rate, the axial temperature gradient is large. Ziegler ${ }^{1-17}$ ), for instance, measured an axial temperature gradient of $1000{ }^{\circ} \mathrm{C} / \mathrm{cm}$ in a neck with a diameter of $2.5 \mathrm{~mm}$. Consequently, the heat flow is mainly in the axial direction, leading to a low value for the radial temperature gradient. This is in agreement with the flat solid-liquid interface (flat freezing isotherm) present in the neck ${ }^{1-17}$ ). In combination with the small dimensions of the neck, it causes the internal stress to remain low. No severe dislocation multiplication will therefore take place. Furthermore, because of the low dislocation mobility in silicon, only those dislocations which terminate at the solid-liquid interface are capable of keeping up with the fast-moving interface. These dislocations will soon reach the surface and disappear. On the other hand dislocations, which are exactly parallel to the direction of crystal growth, may persist. In the case of growth exactly in the $\langle 111\rangle$ or $\langle 100\rangle$ direction it appeared that the residual dislocations, which were not parallel to these lowindex directions, often become aligned along the growth direction ${ }^{1-17,18}$ ). For $\langle 111\rangle$ crystals it was found ${ }^{1-17}$ ) that the dislocations are more readily eliminated if the growth direction is taken a few degrees out of this orientation.

After fast growth over a few centimetres the neck usually becomes dislocation-free. The diameter of the crystal can then be enlarged by lowering the growth rate and the melt temperature. The crystal stays dislocation-free as long as strong disturbances such as melt vibrations, excessive radial temperature gradients or solid connections between the crystal and the pedestal are prevented. In this way C.Z. as well as F.Z. dislocation-free silicon crystals can be grown.

\subsection{Point defects in melt-grown silicon crystals}

\subsubsection{Impurities in silicon}

In silicon crystals grown from the melt a variety of point defects are present in the lattice after the material is cooled down to room temperature. Two kinds of point defects can be distinguished: (1) chemical impurities will be present, either intentionally added (dope) or unwanted, (2) thermally generated point defects such as vacancies and interstitials. The concentrations of unwanted impurities, such as oxygen, carbon and metals, present in C.Z. and F.Z. silicon as well as methods for their detection will now be discussed successively.

(a) Oxygen. Several measuring techniques have been developed to determine the oxygen concentration $\left(N_{\mathrm{ox}}\right)$ in silicon. The technique most widely used to- 
day is the infrared-absorption method ${ }^{1-19}$ ). Oxygen atoms mainly occupy interstitial sites in the lattice. These atoms give rise to the formation of several absorption bands in the infrared spectrum, that at $9 \mu \mathrm{m}$ wavelength being dominant. Kaiser et al. ${ }^{1-19}$ ) were able to plot a calibration curve for the absorption coefficient at $9 \mu \mathrm{m}$ as a function of oxygen concentration by combining infrared measurements with vacuum fusion analysis. The sensitivity of the infrared method can be increased by cooling the sample. Pajot ${ }^{1-20}$ ) estimated the detection limit to be about $5.10^{14} \mathrm{at} / \mathrm{cm}^{3}$ at $8 \mathrm{~K}$.

The value of $N_{\mathrm{ox}}$ can also be determined by means of an ion-drift technique, in which the effective drift mobility of lithium ions is measured in a reversebiased $p-n$ junction ${ }^{1-21}$ ). The detection limit is claimed to be about $10^{15} \mathrm{at} / \mathrm{cm}^{3}$.

Finally, $N_{\text {ox }}$ can be measured by a-activation analysis ${ }^{1-22}$ ). Silicon is irradiated with $\alpha$-particles causing the reaction: ${ }^{16} \mathrm{O}(\alpha, p){ }^{18} \mathrm{~F} ; N_{\text {ox }}$ can be calculated from the $\beta^{+}$activity of the ${ }^{18} \mathrm{~F}$ isotopes. The theoretical detection limit is about $5.10^{13}$ at $/ \mathrm{cm}^{3}$.

Oxygen concentrations in the range $10^{14}-2 \cdot 10^{18} \mathrm{~cm}^{-3}$ have been measured in the different types of crystals. The oxygen concentrations, measured with the various methods, were in reasonable agreement with one another, although the infrared method seems to give somewhat lower values ${ }^{1-22}$ ).

The oxygen concentration depends on the crystal-growth conditions. It will be clear that the amount of this impurity in the polycrystalline starting material plays an important part, as well as the partial pressure of oxygen in the atmosphere used. Growth under high-vacuum conditions gererally leads to lower values of $N_{\text {ox }}$ as compared with crystal growth in a gas atmosphere because of the low partial pressure of oxygen in high vacuum. Furthermore, vacuum favours the evaporation of oxygen out of the melt in the form of $\mathrm{SiO}^{1-23}$ ). In C.Z. silicon $N_{\mathrm{cx}}$ is strongly influenced by the rotation rates of the crystal and the crucible ${ }^{\mathbf{1 - 2 4 , 2 5}}$ ). Fast rotation causes an intensive stirring of the melt. This increases the rate at which crucible material is dissolved into the melt. The oxygen concentrations which are present in C.Z. silicon crystals are found to vary between about $10^{17}$ and $2 \cdot 10^{18}$ at $/ \mathrm{cm}^{3-19,22}$ ). The latter value equals the maximum solubility of oxygen in silicon at the melting point ${ }^{1-24}$ ). As was to be expected lower oxygen concentrations have been measured in F.Z. silicon. Typical values for this material are $10^{14}$ to $10^{16} \mathrm{at} / \mathrm{cm}^{3}{ }^{1-20.22}$ ), the lower values refer to vacuum-grown crystals.

(b) Carbon. Carbon atoms occupy substitutional positions in the silicon lattice ${ }^{1-26}$ ). Because these impurity atoms give an absorption band in the infrared transmission spectrum at a wavelength of $16.6 \mu \mathrm{m}$, an absorption technique similar to that of oxygen can be used to measure the carbon content ${ }^{1-26}$ ). Other techniques available for carbon detection are chemical analysis ${ }^{1-27}$ ), lattice-parameter measurements with multiple crystal X-ray spectrometry ${ }^{1-28}$ ) 
or $\alpha$-activation analysis ${ }^{1-22}$ ). Gross et al ${ }^{1-22}$ ) found carbon concentrations in C.Z. silicon between $4,10^{16}$ and $5 \cdot 10^{17}$ at $/ \mathrm{cm}^{3}$ using $\alpha$ activation and infrared spectroscopy. The concentration in F.Z. crystals varies between 2. $10^{15}$ and $10^{17}$ at $/ \mathrm{cm}^{3} 1-22,26,28$ ). Very high values were found both for C.Z. and F.Z. material by Schink ${ }^{1-27}$ ) using wet chemical methods (3-9. $\left.10^{18} \mathrm{at} / \mathrm{cm}^{3}\right)$. It appears, however, that this method for carbon detection is less reliable ${ }^{1-22}$ ).

(c) Other impurities. The concentration of metallic impurities in as-grown crystals has been measured by, for example, neutron-activation analysis, mass spectrometry and chemical methods. Under clean growth conditions the concentrations of metallic impurities remain below the detection limit of the abovementioned methods $\left(\approx 10^{13} \mathrm{~cm}^{-3}\right)$. These low concentrations are mainly due to the purity of the polycrystalline starting material and the low values of the distribution coefficients of metals in the silicon solid-liquid system ${ }^{1-29}$ ).

The impurity concentrations generally present in silicon are summarized in table 1-I.

\section{TABLE 1-I}

Typical impurity concentrations in silicon (at $\left./ \mathrm{cm}^{3}\right)$

\begin{tabular}{l|l|l|c}
\hline & oxygen & \multicolumn{1}{|c}{ carbon } & metals \\
\hline C.Z. Si & $10^{17}-2 \cdot 10^{18}$ & $4 \cdot 10^{16}-5 \cdot 10^{17}$ & $<5.10^{13}$ \\
F.Z. Si & $10^{14}-10^{16}$ & $2 \cdot 10^{15}-10^{17}$ & $<5.10^{13}$ \\
\hline
\end{tabular}

Since impurities exhibit a retrograde solubility in silicon ${ }^{1-29}$ ), the as-grown material becomes supersaturated with oxygen and carbon on cooling. Supersaturation with metallic impurities will be negligible because of their low concentrations. Supersaturation of F.Z. silicon with oxygen and carbon will occur at low temperatures only. For an oxygen concentration of $10^{15} \mathrm{at} / \mathrm{cm}^{3}$ this will happen at about $300^{\circ} \mathrm{C}$, as follows from an extrapolation of the solubility curve given by Trumbore $\left.{ }^{1-29}\right)$. As the diffusion coefficient of oxygen is extremely low at this temperature viz. $\left.D\left(300^{\circ} \mathrm{C}\right) \approx 1 \cdot 5 \cdot 10^{-29} \mathrm{~cm}^{2} / \mathrm{s}^{1-30}\right)$, precipitation of oxygen will not occur. The same applies to carbon in F.Z. silicon. C.Z. crystals, however, become supersaturated with oxygen and carbon at relatively high temperatures. Consequently, precipitation can be expected to take place during growth. This precipitation process proceeds during annealing treatments at elevated temperatures ${ }^{1-31,32,33}$ ).

Precipitation of deliberately added dope impurities such as phosphorus, arsenic, antimony or boron will not take place at the dope levels usually involved in semiconductor-device technology. In heavily boron-doped crystals, 
with a level of doping of about $10^{20} \mathrm{~cm}^{-3}$ and grown by the C.Z. method, precipitation has been detected ${ }^{1-34}$ ).

In conclusion it can be said that precipitation of impurities will be negligible in F.Z. silicon, whereas oxygen and carbon precipitation may occur in C.Z. silicon.

\subsubsection{Impurity striations}

The concentration $\left(N_{\mathrm{s}}\right)$ of an impurity incorporated in a growing crystal depends on the concentration of this impurity in the melt $\left(N_{\mathrm{L}}\right)(\mathrm{S}=$ solid, $\mathrm{L}=$ liquid). The ratio $N_{\mathrm{s}} / N_{\mathrm{L}}$, called the effective distribution coefficient ( $K_{\mathrm{erf}}$ ), has been calculated by Burton et al. ${ }^{1-35}$ ). Assuming a planar solid-liquid interface and neglecting diffusion in the solid, the following expression was derived:

$$
K_{\text {eff }}=\frac{N_{\mathbf{s}}}{N_{\mathrm{L}}}=\frac{K}{K+(1-K) \exp \left(-V_{g} \delta / D\right)},
$$

where $K$ is the equilibrium distribution coefficient as determined by the siliconimpurity phase diagram, $V_{\theta}$ is the crystal-growth rate, $\delta$ the thickness of a layer in the melt adjacent to the solid-liquid interface where solute transport takes place solely by diffusion (about $10^{-2} \mathrm{~cm}$ ), and $D$ the diffusion coefficient of the impurity in the melt $\left(10^{-4}-10^{-5} \mathrm{~cm}^{2} / \mathrm{s}\right)$. The reported values of $K$ are smaller than unity for all important impurities in silicon ${ }^{1-29}$ ). Taking, for instance, $K$ equal to $0 \cdot 1, D=10^{-4} \mathrm{~cm}^{2} / \mathrm{s}$ and a growth rate of $5.10^{-3} \mathrm{~cm} / \mathrm{s}$, as is frequently used, we obtain: $K_{\text {eff }}=1.6 \mathrm{~K}$.

As mentioned in secs 1.2 .1 and 1.2.2, silicon crystals are rotated during growth. Although this reduces thermal asymmetries in the growth system, a perfectly cylindrical symmetric temperature distribution around the axis of rotation is generally not achieved. Consequently, every point on the solidliquid interface will tend to oscillate between hotter and cooler parts of the temperature field during rotation. This results in a periodic variation in growth rate. Because of this the impurity concentrations will exhibit a periodic variation along the crystal-growth direction (eq. (1.1)). The crystal area grown at the largest growth rate will have the form of a spiral ramp with a pitch equal to the pulling rate divided by the rotation rate. This area will contain the highest impurity concentration because $N_{\mathrm{s}}$ increases with increasing growth rate (eq. $(1.1), K<1$ ). The region in between this spiral ramp has been solidified more slowly and will contain a lower impurity concentration. In a longitudinal crystal section parallel to the crystal axis the regions of high and low impurity content will form a system of narrow bands more or less parallel to the solid-liquid interface. These bands are called impurity striations. When the impurity concentration is high as, for instance, in 
heavily doped material, these striations can be revealed by means of several techniques such as preferential etching ${ }^{1-36,37,38.39}$ ), electrolytic copper plating ${ }^{1-40}$ ) and X-ray topography ${ }^{1-40,41}$ ). On a crystal cross-section the detection techniques, mentioned above, reveal a spiral pattern ${ }^{1-38,39.34,40,43}$ ) which is consistent with the helical distribution of impurities.

\subsubsection{Vacancies and interstitials in silicon crystals}

1.3.3.1. Equilibrium concentrations of vacancies and interstitials

Vacancies and interstitials are thermodynamically stable defect configurations at elevated temperatures because their formation lowers the free energy of the crystal. The entropy is increased by the introduction of these point defects and this compensates at relatively high temperatures for the considerable energy (enthalpy) required for their formation. The energies of formation of both types of point defects, however, are not accurately known. Theoretical estimates have resulted in values between 2.3 and $4.6 \mathrm{eV}^{1-44}$ ) for the vacancyformation energy $E_{\mathbf{V}} \mathbf{F}$. The formation energy of an interstitial $E_{\mathbf{l}} \mathbf{F}$ is even less accurately known but it is assumed to be higher than $\left.E_{\mathrm{V}}{ }^{1-45}\right)$.

The equilibrium concentration of both vacancies $\left(N_{\mathrm{v}}\right)$ and interstitials $\left(N_{1}\right)$ will be governed by Boltzmann statistics:

$$
N_{\mathbf{v}}=N \exp \left(-A G_{\mathbf{v}} / k T\right)=N \exp \left(\Delta S_{\mathbf{v}} / k\right) \exp \left(-E_{\mathbf{v}} \mathbf{F} / k T\right)
$$

and

$$
N_{\mathrm{I}}=N^{\prime} \exp \left(-\Delta G_{\mathrm{l}} / k T\right)=N^{\prime} \exp \left(\Delta S_{\mathrm{l}} / k\right) \exp \left(-E_{\mathrm{l}}^{\mathrm{F}} / k T\right),
$$

where $N$ and $N^{\prime}$ are the number of atomic and interstitial sites, $\Delta G_{\mathrm{V}}$ and $\Delta G_{\mathrm{I}}$ are the free energies of formation of a vacancy and an interstitial and $\Delta S_{\mathrm{V}}$ and $\Delta S_{\mathrm{I}}$ are the entropies of formation of a vacancy and an interstitial, respectively. The exact values for $\Delta G_{\mathrm{v}}$ and $\Delta G_{\mathrm{I}}$ are not known. It is well known, however, that the equilibrium concentration of vacancies in f.c.c. and b.c.c. metals is much higher than that of interstitials because $\Delta S_{\mathrm{V}}$ and $\Delta S_{\mathrm{I}}$ are both about equal to $k$, while $E_{\mathrm{V}} \mathbf{F}<E_{\mathrm{I}} \mathrm{F}$. This difference in formation energy is due to the lattice strain introduced when self-interstitials, which do not fit in the interstitial sites, are generated. The introduction of vacancies is not accompanied by such strain effects. Therefore we have for these metals $\Delta G_{1}>\Delta G_{\mathrm{V}}$ and thus $N_{\mathrm{I}} \ll N_{\mathrm{v}}$. For similar reasons it is generally believed that the condition $N_{\mathrm{I}} \ll N_{\mathrm{V}}$ also applies to materials like silicon. Therefore it is assumed that the influence of thermally generated point defects on the crystal properties is dominated by the vacancies.

From thermodynamical considerations Seeger and Swanson ${ }^{1-46}$ ) estimated the upper limit for the equilibrium concentration of vacancies at the melting point to be $9.10^{15} \mathrm{~cm}^{-3}$, and the lower limit $1 \cdot 2 \cdot 10^{13} \mathrm{~cm}^{-3}$. 
Most experimental methods used for the determination of vacancy concentrations at high temperatures require quenching treatments from the temperatures of interest to room temperature or lower. Bemski and Dias ${ }^{1-47}$ ) and Elstner and Kamprath ${ }^{1-48}$ ) reported on quenching experiments of this kind on silicon. In both investigations donor-type defects were introduced by the quenching procedure. The material used by Elstner et al. ${ }^{1-48}$ ) was $p$-type F.Z. silicon grown in vacuum with a dislocation density below $2.10^{4} \mathrm{~cm}^{-2}$. After quenching from temperatures between 750 and $1025^{\circ} \mathrm{C}$ the defect concentration as a function of temperature was obtained from Hall measurements. The formation energy of the defects, which were thought to be either vacancy-oxygen complexes or vacancy clusters, was estimated to be $2.5 \mathrm{eV}$. Extrapolation of their results to $1420^{\circ} \mathrm{C}$ results in a vacancy concentration of about $2 \cdot 10^{17} \mathrm{~cm}^{-3}$. Boltaks and Budarina ${ }^{1-49}$ ) recently reported on measurements of the relative change in density of silicon after quenching from temperatures between 950 and $1200^{\circ} \mathrm{C}$. From the decrease in density they calculated vacancy concentrations in this temperature range. Extrapolation of their results gives the very high vacancy concentration of $3.10^{19} \mathrm{~cm}^{-3}$ at $1420^{\circ} \mathrm{C}$. In some $p$-type silicon samples, however, they found an increase in density after quenching, which was attributed to the formation of defect complexes. In general the interpretation of quenching experiments on silicon is difficult for a variety of reasons ${ }^{1-44}$ ). One notable reason is that vacancies are mobile in silicon at room temperature (sec. 1.3.3.2) and form complexes with other vacancies or impurities (sec. 1.3.3.3). Single vacancies will therefore not be present in silicon at room temperature but will have disappeared at sinks or have formed defect complexes.

It will be clear from the foregoing that large discrepancies exist between theoretical estimates and experimental determinations of vacancy concentrations in silicon.

\subsubsection{The mobility of vacancies in silicon}

From low-temperature E.P.R. studies of electron-irradiated silicon it could be concluded that monovacancies in silicon exist in different charge states ${ }^{1-50}$ ): singly positive, neutral, singly negative and doubly negative. The mobility of some of these vacancies has been determined by Watkins ${ }^{1-51,52}$ ) with the aid of E.P.R. measurements in combination with low-temperature annealing treatments. He found that the migration energy depends on the charge state and the material resistivity. The doubly negatively charged vacancy, for instance, has a migration energy of $0.18 \pm 0.02 \mathrm{eV}$ in low-resistivity $n$-type material and anneals out between 70 and $85 \mathrm{~K}$. The vacancy with the odd-electron charge state anneals in $p$-type silicon between 150 and $180 \mathrm{~K}$ with a migration energy of $0.33 \pm 0.03 \mathrm{eV}$. These results are consistent with Hall-coefficient measurements on $\gamma$-irradiated F.Z. $p$-type silicon reported by Matsui and Hasi- 
guti ${ }^{1-53}$ ), as well as with infrared-absorption measurements on electronirradiated material carried out by Ramdas and Fan ${ }^{1-54}$ ).

For the diffusion coefficient of vacancies $\left(D_{\mathrm{v}}\right)$ we have $\left.{ }^{1-58}\right)$ :

$$
D_{\mathrm{v}}(T)=g a^{2} v \exp \left(-E_{\mathrm{v}}{ }^{\mathrm{M}} / k T\right),
$$

where $g$ is a geometrical factor ( $1 / 8$ for the diamond lattice), $a$ the elementary jump distance $\left(2 \cdot 35 \cdot 10^{-8} \mathrm{~cm}\right), \nu$ the apparent jump frequency and $E_{\mathrm{V}}{ }^{\mathrm{M}}$ the migration energy of a vacancy. For $v$ we take the value obtained by Watkins ${ }^{1-52}$ ) $\left(2.10^{12} \mathrm{~s}^{-1}\right)$. For the vacancy with the odd-electron charge state $\left(E_{\mathrm{v}}{ }^{\mathrm{M}}=\right.$ $0.33 \mathrm{eV})$ we obtain at the melting point $\left(T_{M}\right): D_{\mathrm{v}}\left(T_{M}\right)=1.4 \cdot 10^{-5} \mathrm{~cm}^{2} / \mathrm{s}$, while for the doubly negatively charged vacancy $\left(E_{\mathrm{v}}{ }^{M}=0.18 \mathrm{eV}\right)$ we have $D_{\mathrm{v}}\left(T_{M}\right)=4 \cdot 1 \cdot 10^{-5} \mathrm{~cm}^{2} / \mathrm{s}$. Due to the low values of the migration energy, $D_{\mathrm{v}}$ decreases only gradually with decreasing temperature.

\subsubsection{Vacancy-impurity-complex formation}

Monovacancies combine readily with other point defects. This is partly due to the different charge states of the vacancies, which lead to a coulomb interaction between vacancies and impurities with a donor or acceptor character.

A variety of vacancy-impurity complexes have been detected. Watkins and Corbett ${ }^{1-56}$ ) studied the phosphorus-vacancy pair (so-called E-centre). This complex anneals out between 150 and $180^{\circ} \mathrm{C}$ with an activation energy of $0.94 \mathrm{eV}$. The vacancy-aluminium pair forms a stable configuration below about $200^{\circ} \mathrm{C}^{1-57}$ ).

Furthermore, extensive study has been devoted to the formation of vacancy-oxygen complexes ${ }^{1-50.57 .59,60}$ ). With the aid of irradiation experiments, E.P.R. measurements and infrared spectroscopy the structure of the vacancy-oxygen pair (so-called A-centre) is now well established. It can be considered as an off-site substitutional oxygen atom. Corbett et al. ${ }^{1-59}$ ) studied the concentration of this defect in irradiated silicon as a function of temperature. They found that the concentration of the A-centres decreased at temperatures above $300^{\circ} \mathrm{C}$. Furthermore, from infrared-absorption spectra, strong indications were obtained that the disappearance of the A-centres is accompanied by the formation of larger complexes consisting of one vacancy and two or three oxygen atoms respectively.

Finally the combination of two monovacancies gives rise to the formation of divacancies, which can be present in the same charge states as those mentioned for monovacancies ${ }^{1-55}$ ). However, they are much less mobile. The singly negatively charged divacancy, for instance, has a migration energy between $1 \cdot 2$ and $1 \cdot 3 \mathrm{eV}^{1-55}$ ). 


\subsubsection{Vacancy clusters in dislocation-free silicon}

As pointed out in sec. 1.1 it can be expected that as a consequence of the absence of dislocations vacancy clusters will be formed during the growth of dislocation-free silicon crystals. The first indications of the presence of such clusters in high-purity dislocation-free floating-zone material grown in helium were obtained by Plaskett ${ }^{1-4}$ ), using the copper-decoration method, originally developed by Dash ${ }^{1-12}$ ) for revealing dislocations. After copper diffusion into longitudinal crystal sections at temperatures between 900 and $1000{ }^{\circ} \mathrm{C}$, followed by quenching of the samples to room temperature, a striated distribution of copper precipitates was detected with infrared microscopy. It was assumed that these precipitates were formed by copper precipitation on vacancy clusters. The striated distribution was thought to be due to a variation in vacancy concentration caused by the usually occurring variation in growth rate (sec. 1.3.2). Abe et al. ${ }^{1-61,62}$ ) investigated dislocation-free floating-zone silicon grown in high vacuum, using techniques such as preferential etching and copper decoration. A microdefect distribution similar to that present in heliumgrown crystals was found. However, ignoring the low oxygen concentration in F.Z. silicon, these authors attributed the defects to precipitation of oxygen.

Dislocation-free crystals, grown either by the C.Z. method or by F.Z. techniques using insufficiently purified polycrystalline starting material, will become supersaturated with vacancies as well as with impurities such as oxygen and carbon during cooling of the as-grown material (sec. 1.3.1). Consequently, a complicated nucleation process will start finally resulting in precipitation of vacancies, oxygen and carbon. Dash ${ }^{1-1,14}$ ) and Schwuttke ${ }^{1-41,42,63}$ ) have studied dislocation-free C.Z. silicon by means of copper decoration, infrared microscopy and X-ray transmission topography. After decoration of longitudinal crystal sections striated distributions of copper precipitates were netected. These precipitates were thought to be formed on $\mathrm{SiO}_{2}$ precipitates present in the as-grown crystals. Using similar techniques Fiermans and Vennik ${ }^{1-64,65,66}$ ) investigated both dislocation-free C.Z. material and dislocation-free low-oxygen-content silicon, which, however, contained relatively high concentrations of carbon. Enhanced concentrations of oxygen and carbon were found at the centres of the copper precipitates by microprobe analysis. This proved that in the crystals investigated copper precipitation occurred on oxygen and carbon precipitates.

Shul'pina et al. ${ }^{1-31,32}$ ) investigated the precipitation of oxygen and carbon in dislocated and dislocation-free C.Z. silicon during annealing treatments between 900 and $1300{ }^{\circ} \mathrm{C}$. In the case of dislocation-free material the oxygen and carbon precipitates were found to be formed in a striated pattern, whereas precipitates in dislocated silicon were found along the dislocations and homogeneously distributed in the lattice. It was suggested that in the absence of line 
defects both homogeneous and heterogeneous precipitation of both impurities takes place. The sites for heterogeneous precipitation are thought to be vacancy clusters. In some dislocation-free C.Z. crystals Batavin ${ }^{1-33}$ ) found a striated pattern of stacking faults after annealing at temperatures between 900 and $1200^{\circ} \mathrm{C}$ in vacuum. The formation of these defects is explained in terms of oxygen precipitation on vacancy clusters.

From all these experimental results the following preliminary conclusions can be drawn.

(1) During silicon-crystal growth from the melt the material may become supersaturated with point defects. When the crystal contains high concentrations of impurities this supersaturation will result in the formation of precipitates. In the absence of dislocations supersaturation with vacancies may occur, finally resulting in the formation of vacancy clusters.

(2) In dislocation-free silicon precipitates are preferably formed in a striated pattern. This pattern is related to the periodic variation in growth rate.

\section{REFERENCES}

1-1) W. C. Dash, in Doremus et al. (eds), Growth and perfection of crystals, John Wiley and Sons, New York, 1958, p. 361.

1-2) A. G. Tweet, J. appl. Phys. 29, 1520, 1958.

$1-3)$ A. G. Tweet, J. appl. Phys. 30, 2002, 1959.

1-4) T. S. Plaskett, Trans. AIME 233, 809, 1965.

1-5) J. Czochralski, Z. phys. Chem. 92, 219, 1918.

1-6) G. K. Teal and E. Buehler, Phys. Rev. 87, 140, 1952.

1-7) P. H. Keck and W. van Horn, Phys. Rev. 91, 512, 1953.

1-8) W. C. Dash, J. appl. Phys. 31, 736, 2275, 1960.

1-9) E. Billıg, Proc. Roy. Soc. A235, 37, 1956.

1-10) P. Penning, Philips Res. Repts 13, 79, 1958.

1-11) F. C. Frank and W. T. Read, Phys. Rev. 79, 722, 1950.

1-12) W. C. Dash, J. appl. Phys. 27, 1193, 1956.

1-13) A. H. Cottre11, Dislocations and plastic flow in crystals, Clarendon Press, Oxford, 1953.

1-14) W. C. Dash, J. appl. Phys. 30, 459, 1959.

1-15) B. Okkerse, Philıps tech. Rev. 21, 340, 1959.

1-16) U. Zimmerli and A. Steinemann, Z. angew. Math. Phys. 16, 555, 1965.

1-17) G. Ziegler, Z. Naturf. 16a, 219, 1961.

1-18) A. Lindegaard Andersen, Thesis, Technical University of Denmark, 1967.

1-19) W. Kaiser, P. H. Keck and C. F. Lange, Phys. Rev. 101, 1264, 1956.

1-20) B. Pajot, Solıd-State Electron. 12, 923, 1969.

1-21) E. M. Pell, J. appl. Phys. 32, 1048, 1961.

1-22) C. Gross, G. Gaetano, T. N. Tucker and J. A. Baker, Recent news paper 33 RNP, J. electrochem. Soc. 116, 248C, 1969.

1-23) W. Kaiser and P. H. Keck, J. appl. Phys. 28, 882, 1957.

1-24) R. H. Logan and A. J. Peters, J. appl. Phys. 28, 819, $1419,1957$.

1-25) W. Kaiser, H. L. Frisch and H. Reiss, Phys. Rev. 112, 1546, 1958.

1-26) R. C. Newmann and J. B. Willis, J. Phys. Chem. Solids 26, 373, 1965.

1-27) N. Schink, Solid-State Elcctron. 8, 767, 1965.

1-28) J. A. Baker, T. N. Tucker, N. E. Moyer and R. C. Buschert, J. appl. Phys. 39, $4365,1968$.

1-29) F. A. Trumbore, Bell Sys. tech. J. 39, 205, 1960.

1-30) R. A. Logan and A. J. Peters, J. appl. Phys. 30, 1627, 1959.

1-31) I. L. Shul'pina, L. V. Lainer, M. G. Mil'vidskıi and E. P. Rashevskaya, Sov. Phys.-Solid State 9, 1291, 1967. 
1-32) I. L. Shul'pina, A. I. Zaslavskii and T. T. Dedegkaev, Sov. Phys.-Solid State 10, 1070, 1968.

1-33) V. V. Batavin, Sov. Phys.-Cryst. 15, 100, 1970.

1-34) G. H. Schwuttke, J. appl. Phys. 34, 1662, 1963.

1-35) J. A. Burton, R. C. Prim and W. P. Slichter, J. electrochem. Soc. 21, 1987, 1953.

1-36) W. D. Edwards, Can. J. Phys. 38, 439, 1960.

1-37) T. F. Ciszek, in R. R. Haberecht and E. L. Kern (eds), Semiconductor silicon, Electrochem. Soc., 1969, p. 156.

1-38) F. Vieweg-Gutberlet, Solid-State Electron. 12, 731, 1969.

1-39) M. Kamper, J. electrochem. Soc. 117, 261, 1970.

1-40) J. A. M. Dickhoff, Phılıps tech. Rev. 25, 195, 1963/64.

1-4 I) G. H. Schwuttke, J. appl. Phys. 33, 2760, 1962.

1-42) G. H. Schwuttke, in J. B. Newkirk and J. H. Wernick (eds), Direct observation of Imperfections in crystals, John Wiley and Sons, New York, 1962, p. 497.

1-43) M. Hart, Proc. Roy. Soc. A309, $281,1969$.

1-44) A. Seeger and K. P. Chik, Phys. Stat. sol. 29, 455, 1968.

1-45) R. A. Swalin, J. Phys. Chem. Solids 18, 290, 1961.

1-46) A. Seeger and M. L. Swanson, in R. R. Hasigutı (ed.), Lattıce defects in semıconductors, Univ. of Tokyo Press, 1968, p. 93.

1-47) G. Bemski and C. A. Dias, J. appl. Phys. 35, 2983, 1964.

1-48) L. Elstner and W. Kamprath, Phys. Stat. sol. 22, 541, 1967.

1-49) B. I. Boltaks and S. I. Budarina, Sov. Phys.-Solid State 11, 330, 1969.

1-50) G. D. Watkins, in P. Baruch (ed.), Radiatıon damage in semıconductors (Internat. Conf. Physics of Semiconductors), Dunod, Paris, 1965, p. 97.

1-51) G. D. Watkins, Symposium on Radiation Effects in Semiconductor Components, Toulouse, 1967.

1-52) G. D. Watkins, J. phys. Soc. Japan 18, 22, 1963, Suppl. II.

$1-53)$ K. Matsui and R. R. Hasiguti, J. phys. Soc. Japan 20, 487, 1965.

1-54) A. K. Ramdas and H. Y. Fan, J. phys. Soc. Japan 18, 33, 1963, Suppl. II.

1-55) G. D. Watkins and J. W. Corbett, Phys. Rev. 138A, 543, 1965.

1-56) G. D. Watkins and J. W. Corbett, Phys. Rev. 134A, 1359, 1964.

1-57) G. D. Watkins, Phys. Rev. 155, 802, 1967.

${ }^{1-58}$ ) L. Kendall and D. B. de Vries, in R. R. Haberecht and E. L. Kern (eds), Semiconductor silicon, Electrochem. Soc., 1969, p. 358.

1-59) J. W. Corbett, G. D. Watkins and R. S. McDonald, Phys. Rev. 135, A1381, 1964.

1-60) J. C. Corelli, G. Oehler, J. F. Becker and K. J. Eisenkraut, J. appl. Phys. 36, $1787,1965$.

1-61) T. Abe, T. Samızo and S. Maruyama, Jap. J. appl. Phys. 5, 458, 1966.

1-62) T. A be and S. Maruyama, Denki Kagaku 35, 149, 1967.

1-63) G. H. Schwuttke, J. electrochem. Soc. 109, 27, 1962.

1-64) L. Fiermans and J. Vennik, Phys. Stat. sol. 12, 277, 1965.

1-65) L. Fiermans and J. Vennik, Phys. Stat. sol. 21, 627, 1967.

1-66) L. Fiermans and J. Vennik, Phys. Stat. sol. 22, 463, 1967. 


\section{METHODS FOR DETECTING MICRODEFECTS IN SILICON CRYSTALS}

\subsection{Introduction}

A number of methods used for the detection of microdefects in silicon crystals have already been mentioned in sec. 1.3.3.4. These methods were: preferential etching, X-ray transmission topography and copper decoration in combination with infrared microscopy. These techniques, some in a slightly modified form, were also used in the present study. This chapter will describe these techniques and also a newly developed decoration technique based on the precipitation properties of lithium in silicon.

\subsection{Preferential etching}

A variety of preferential etchants have been developed for revealing defect structures on silicon-crystal surfaces. Crystal imperfections present at the surface give rise to the formation of etch pits because the etching rate is locally enhanced by the lattice strain around these imperfections. Thus the presence and concentration of defects near the surface can be determined, while conclusions concerning the character of the defects can be drawn from the form of the etch pits.

The preferential etchant, developed by Sirtl and Adler ${ }^{2-1}$ ) and consisting of a mixture of $\mathrm{CrO}_{3}, \mathrm{HF}$ and $\mathrm{H}_{2} \mathrm{O}$ is most widely used today for the detection of extended defects such as dislocations and stacking faults. However, because of the extreme sensitivity of this etchant for small local strain fields, it can also be used for the study of microdefects ${ }^{2-2,3}$ ). In the present study this etchant was used in a modified form as compared with the composition given by Sirtl et al. ${ }^{2-1}$ ), mainly in order to reduce the etching rate. This permits better control of the etching process. The following composition was used: HF (49\%): $\mathrm{CrO}_{3}(33 \%$ aqueous solution $)=1: 2$, giving an etching rate lower than $1 \mu \mathrm{m} / \mathrm{min}$.

\subsection{The copper-decoration technique}

Imperfections in crystals which are transparent to visible and to infrared radiation can be made observable when a suitably chosen impurity is precipitated on them. This method has been called decoration and is mainly used for revealing dislocations. The opaque precipitates arranged along a dislocation line make the latter detectable in either the optical or the infrared microscope. Decorated dislocations have been observed in silver halides ${ }^{2-4}$ ), alkali halides $^{2-5}$ ), germanium ${ }^{2-6}$ ) and silicon ${ }^{2-7}$ ).

For silicon a valuable decoration technique has been developed by Dash ${ }^{2-7}$ ) using copper as the precipitating impurity. Although it has been established 
that one can also decorate dislocations in silicon with the aid of other impurities such as aluminium ${ }^{2-8}$ ), nickel ${ }^{2-9,10,11}$ ), iron ${ }^{2-12}$ ) and recently lithium $^{2-13}$ ), copper is nearly exclusively used in investigations of crystal imperfections. This is mainly due to the relative simplicity of the technique ${ }^{2-14 \ldots 24}$ ).

Copper is usually diffused into a silicon slice at a temperature between 950 and $1200{ }^{\circ} \mathrm{C}$. After saturation, leading to copper concentrations of $10^{17}-10^{18} \mathrm{~cm}^{-3}$, the crystal sample is cooled to room temperature. Because copper in silicon exhibits a strongly retrograde solubility ${ }^{2-25}$ ) the silicon becomes supersaturated with copper during cooling. Since the band gap of silicon semiconductor material is $1.11 \mathrm{eV}$ this material is transparent for wavelengths above $1 \cdot 1 \mu \mathrm{m}$ as long as free-carrier absorption remains low (low dope level). This enables investigation of the decorated samples in the infrared microscope. Dislocations as well as oxygen and carbon precipitates have been detected in this way ${ }^{2-14 \ldots 24}$ ).

Copper diffusion is usually carried out by heating etched silicon samples covered with $\left.\mathrm{Cu}\left(\mathrm{NO}_{3}\right)_{2}{ }^{2-7,22}\right)$ or with an electrolytically deposited copper layer ${ }^{2-19}$ ). Sometimes even copper powder is used ${ }^{2-21}$ ). The disadvantage of these methods is the necessity of lapping and polishing the samples afterwards in order to remove the alloyed surface layer. For the present study a new decoration system was developed in which contamination of the sample surface is prevented (fig. 2.1). This system consists of a resistance-heated sintered molybdenum susceptor (1) placed in a small quartz vessel (2). A copper wire (4) in the vertical tube (3) can be heated electrically and acts as the copper source.

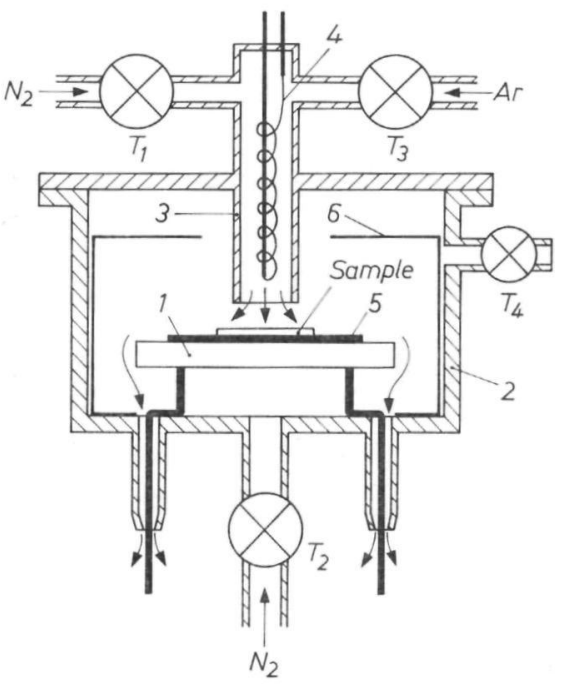

Fig. 2.1. The set-up used for copper decoration. Mo-susceptor (1); silica vessel (2); copper source $(3,4)$; silicon slice (5); Mo-reflector (6). Taps $T_{1}, T_{2}, T_{3}$ and $T_{4}$. 
The decoration procedure is as follows. The sample, which is usually in the form of a sawn slice with a thickness of about $350 \mu \mathrm{m}$, is non-preferentially etched on both sides prior to decoration in order to remove the surface damage caused by sawing. Then the sample is placed on the thin silicon slice (5) which covers the susceptor. This silicon slice prevents contamination of the back of the sample. The vessel (2) is now closed and the system is flushed with nitrogen via taps $T_{1}, T_{2}$ and $T_{4}$. These taps are then closed, $T_{3}$ is opened and an argon flow is sent through the tube (3) and leaves the system at the bottom (arrows). The susceptor is now heated until the sample reaches the desired diffusion temperature, which is measured with an optical pyrometer. This temperature was $950{ }^{\circ} \mathrm{C}$ in most experiments. Temperature gradients in the system are strongly reduced by means of a molybdenum heat reflector (6). This prevents plastic deformation of the samples. Copper is evaporated into the argon flow by heating the wire (4), resulting in copper diffusion into the sample. After about 30 minutes a homogeneous distribution of copper in the sample will be obtained, whereupon the source can be switched off. Finally, the whole system is cooled down to room temperature by switching off the heating current of the susceptor and flushing the vessel with a nitrogen-argon mixture. The cooling rate of the sample should not be too small because then large amounts of copper are lost for decoration by out-diffusion to the surface of the sample. On the other hand an extremely fast quenching will prevent the diffusionlimited copper precipitation on the various lattice imperfections. Good results were obtained with cooling rates of about $100^{\circ} \mathrm{C} / \mathrm{s}$. In this set-up the temperature and the available amount of copper can be regulated independently. Alloying phenomena at the sample surface can be prevented by choosing the correct combination of these two parameters. This combination is established empirically. Under these conditions the sample can be investigated immediately after decoration.

The infrared system used for visual examination of the decorated samples consisted of a closed-circuit television set adapted to an optical transmission microscope. The television camera was provided with an infrared-sensitive plumbicon tube containing a $\mathrm{PbO}-\mathrm{PbS}$ target. Photographs of the samples, however, were made using an infrared microscope type NV-II B from Nippon Electric Company Ltd.

\subsection{The lithium-decoration technique}

Apart from a copper-decoration method a method for the decoration of microdefects with lithium was developed. For reasons to be discussed in this section, it was expected that lithium decoration would give additional information about grown-in defects in silicon.

Lithium atoms in solid solution in silicon occupy interstitial positions in the lattice. Because of this, and in combination with the small size of these im- 
purity atoms $(0.68 \AA)$, the diffusion coefficient of lithium is high even at relatively low temperatures $\left.\left(D=2 \cdot 4 \cdot 10^{-3} \exp (0 \cdot 65 \mathrm{eV} / k T)\right)^{2-26}\right)$.

Moreover the solubility of lithium in silicon is about three orders higher than that of copper ${ }^{2-27}$ ). Consequently, high concentrations of lithium can be introduced by diffusion at low temperatures (e.g. $8 \cdot 10^{17} \mathrm{~cm}^{-3}$ at $\left.400{ }^{\circ} \mathrm{C}\right)^{2-27}$ ) in reasonably short times (a few hours). Just like copper, lithium exhibits a retrograde solubility in silicon ${ }^{2-27}$ ), causing supersaturation during cooling after in-diffusion. This will result in lithium precipitation.

Several studies have been made on the character of the nucleation sites for lithium precipitation ${ }^{2-27 \ldots 31}$ ). It has been established that only heterogeneous nucleation processes occur. Using the transmission electron microscope Weltzin ${ }^{2-28}$ ) detected lithium precipitation on stacking faults. Ferman et al. $^{2-29,30}$ ) studied lithium precipitation in C.Z. and F.Z. silicon. From $\gamma$-irradiation experiments using silicon with various oxygen concentrations they obtained strong indications that vacancy-oxygen pairs (A-centres) formed preferred nucleation sites for lithium. These results are in good agreement with results reported by R. C. Young et al. ${ }^{2-31}$ ). Recently Takabatake et al. ${ }^{2-17}$ ) were able to decorate dislocations in F.Z. crystals with lithium.

We have studied the lithium precipitation in F.Z. material for several purposes (see also chapters 3 and 5). One of the aims was to investigate whether microdefects, such as vacancy clusters, could be decorated with this metal. As pointed out, this would require much lower diffusion temperatures than those necessary for copper. One of the advantages of such low-temperature decoration might be that microdefects which are not stable at higher temperatures will now become detectable, whereas the chance to create additional microdefects at, for instance, $400{ }^{\circ} \mathrm{C}$ is much smaller than that at the temperature required for copper decoration. Furthermore, defects which do not act as nucleation sites for copper may do so for lithium.

The set-up used for lithium diffusion is shown schematically in fig. 2.2. The silica tube (1) can be closed with lid (2). The quartz support (3) and the small molybdenum box (4) are connected to this lid via tube (5) and wires (6), re-

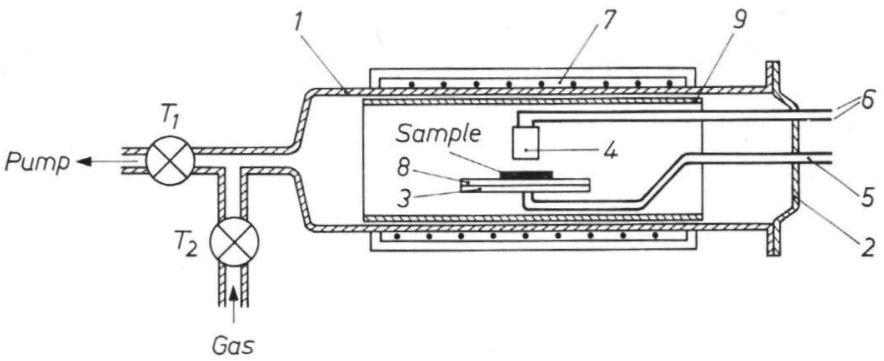

Fig. 2.2. The set-up used for lithium diffusion. Silica tube (1); lid (2); silica support $(3,5)$; lithium source $(4,6)$; tubular furnace (7); Mo-disk (8); silica inner tube (9). Taps $T_{1}$ and $T_{2}$. 
spectively. The tube can be evacuated down to a pressure of about $10^{-3}$ Torr via tap $T_{1}$ or filled with gas via tap $T_{2}$. Furthermore, the tube can be heated by means of the furnace (7), while the temperature is measured with a chromel-alumel thermocouple located in tube (5).

The lithium-diffusion procedure is as follows. The box (4) is filled with a few $\mathrm{mm}^{3}$ of lithium and the sample to be investigated is placed on the molybdenum disk (8) covering the support (3). The sample has been polished or etched in advance, to ensure a damage-free surface. The tube ( 1 ) is closed and evacuated, then lithium is evaporated on to the top side of the silicon sample by heating the box (4) electrically. Subsequently the sample is heated up to the required diffusion temperature by means of the tubular furnace (7) so that lithium diffuses into the silicon from the alloyed layer formed at the surface of the sample. The diffusion process is carried out in vacuum. Because lithium reacts with silica at the temperatures used, the tube (1) is protected by the silica inner tube (9), which is cleaned in HF prior to the diffusion procedure, whereas the disk (8) prevents chemical attack on the support (3). After the diffusion process has been completed, tube ( $\mathrm{I}$ ) is filled with argon, the lid removed and the sample dropped on to a stainless-steel heat sink. This enables the sample to be quenched to room temperature within a few seconds.

After quenching the silicon will be supersaturated with lithium. If the sample is subsequently annealed at temperatures at which reasonable lithium diffusion is possible, precipitation will take place. An annealing temperature of $160{ }^{\circ} \mathrm{C}$ has been chosen (see 3.5). The annealing treatments are carried out in a simple tube furnace in a nitrogen atmosphere.

Lithium in solid solution in silicon acts as a shallow donor, singly ionized at room temperature (energy level $0.033 \mathrm{eV}$ below the conduction band), whereas precipitated lithium is not electrically active. Consequently, the amount of lithium present after diffusion and quenching, as well as the amount which precipitates during annealing, can be determined by measuring the electrical resistivity. This was done using the standard four-point-probe technique ${ }^{2-32}$ ). From these measurements the total concentration of nucleation sites for lithium can be calculated (sec. 4.4). In addition to the electrical method the precipitation and decoration processes have been studied with the infrared microscope and in particular with the $\mathrm{X}$-ray transmission topographical technique.

\subsection{X-ray transmission topography}

A large number of $\mathrm{X}$-ray topographical techniques are available to-day for the investigation of lattice imperfections in nearly perfect crystals. The topographs can be made either in reflection (Bragg geometry), where the incident and diffracted beams enter and leave by the same crystal surface, or in transmission (Laue geometry), where these beams enter and leave by different crystal surfaces. Imperfections, present in the crystal, such as dislocations or precipi- 
tates, are recorded on these topographs because the lattice distortion around the defects influences the diffracted X-ray intensity as will be discussed later in this section.

In the present study the transmission scanning method by Lang ${ }^{2-33}$ ) was used. Figure 2.3 shows the horizontal arrangement of the Lang method. A

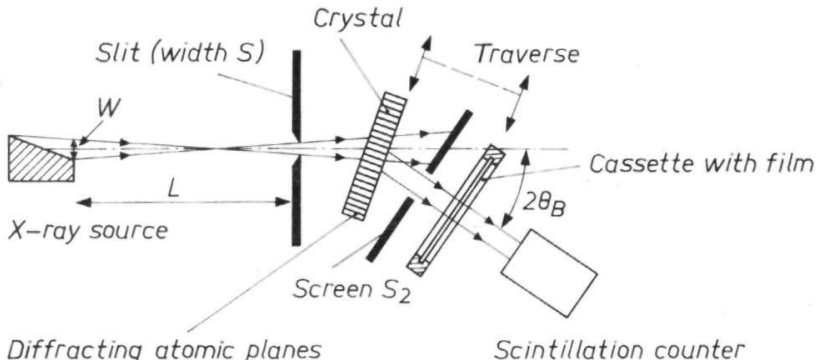

Fig. 2.3. Horizontal arrangement of the scanning X-ray transmission topographical technique by Lang.

beam of X-rays emitted from an X-ray source (width $W$, height $H$ ) passes through a system of slits (width $S$ ). It is then diffracted through the crystal slice to be investigated. The intensity of the diffracted beam is measured with a scintillation counter set at an angle $2 \theta_{\mathbf{B}}$, where $\theta_{\mathbf{B}}$ is the Bragg angle for the set of diffracting atomic planes used. The crystal is oriented in such a way that the counter indicates the maximum diffracted intensity. A cassette, containing an X-ray-sensitive film, is placed between the counter and the crystal. The direct beam is absorbed by a screen $\left(\mathrm{S}_{2}\right)$ inserted between the crystal and the film, while the diffracted beam reaches the photographic film via a slit in screen $\mathrm{S}_{2}$. As we shall see in this section, the slit width $S$ is generally fairly small, which means that with this arrangement a narrow ribbon-shaped vertical region of the crystal is imaged on the film. Simultaneous traversing of the crystal and film in the plane of the crystal slice enables a large area of the sample to be recorded. The total number of scans, determining the exposure time, depends on the diffracted-beam intensity and the film sensitivity. In this way a one-to-one transmission topograph is obtained.

The characteristic $\mathrm{K} \alpha$ radiation of the $\mathrm{X}$-ray spectrum is generally used. Because of the doublet structure of this line, double imaging will generally occur. It can, however, be prevented by making the horizontal beam divergence $\Phi$ smaller than the difference in diffraction angle $\Delta \theta$ between the $\mathrm{K} \alpha_{1}$ and $\mathrm{K} \alpha_{2}$ components. From the Bragg reflection condition

we derive

$$
2 d_{h k l} \sin \theta_{\mathbf{B}}=\lambda
$$

$$
\Delta \theta=\frac{\Delta \lambda_{\alpha_{1}, \alpha_{2}}}{2 d_{h k l} \cos \theta_{\mathrm{B}}},
$$


where $d_{h k l}$ is the spacing of the atomic planes $(h k l)$ used for diffraction and $\lambda$ the wavelength. Using MoK $\alpha$ radiation, for example, this means that for low index planes the horizontal beam divergence may amount to a few minutes of arc. For a (111) reflex $\Delta \theta$ equals $2 \cdot 8^{\prime}$. For the divergence we find (see fig. 2.3):

$$
\Phi=\frac{W+S}{L} .
$$

Taking $L \approx 50 \mathrm{~cm}$ double imaging by the $K \alpha_{1}$ and $K \alpha_{2}$ reflections at (111) planes will be prevented, provided $W+S$ is less than about $0.35 \mathrm{~mm}$.

Two different theories, the kinematical and the dynamical, exist with which the X-ray-intensity distribution in the topographs can be understood. The chief difference between them is that the kinematical theory neglects the interference between the incident beam and multiple-reflected beams inside the crystal. Because this is permissible only in very thin crystals (thickness $t^{\prime} \approx 1 \mu \mathrm{m}$ ), the X-ray diffraction in nearly perfect crystals with thicknesses in the range normally used for topography $\left(t^{\prime}>50 \mu \mathrm{m}\right)$ is better described by the dynamical theory.

As is discussed before, the horizontal divergence $\Phi$ of the incident beam is a few minutes of arc. According to the dynamical diffraction theory ${ }^{2-34}$ ), however, only a small portion of the incident beam is diffracted by a perfect crystal, while the main part leaves the crystal in the original direction (fig. 2.4).

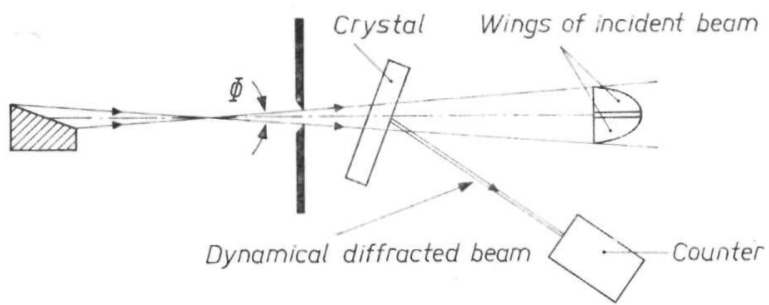

Fig. 2.4. Direct-image-contrast formation by crystal imperfections in transmission topography.

If the crystal contains defects such as dislocations or precipitates, the situation is different. The lattice planes around these imperfections will be bent, causing additional diffraction of X-rays from the wings of the incident beam. This means an X-ray-intensity increase caused by these defects. Consequently, the final topograph will show black images of the defects (kinematical or direct image) superimposed on a grey dynamical background. The excess diffracted $\mathrm{X}$-rays originating from the defects will be partly absorbed before leaving the crystal due to the normal photoelectric-absorption process. Thus the direct 
image intensity will decrease exponentially with $\exp \left(-\mu t^{\prime}\right), \mu$ being the photoelectric-absorption coefficient. Consequently, a reasonable direct-image contrast is obtained for thin slices only $\left(\mu t^{\prime} \leqslant 1\right)$.

In this study nearly exclusively silicon samples with $\mu t^{\prime}<1$ are used. Figure 2.5 is an example of a transmission X-ray topograph of a sample exhibiting direct-image contrast. The photograph shows a cross-sectional slice cut from a silicon crystal with a high dislocation density and some impurity precipitates in the crystal centre.

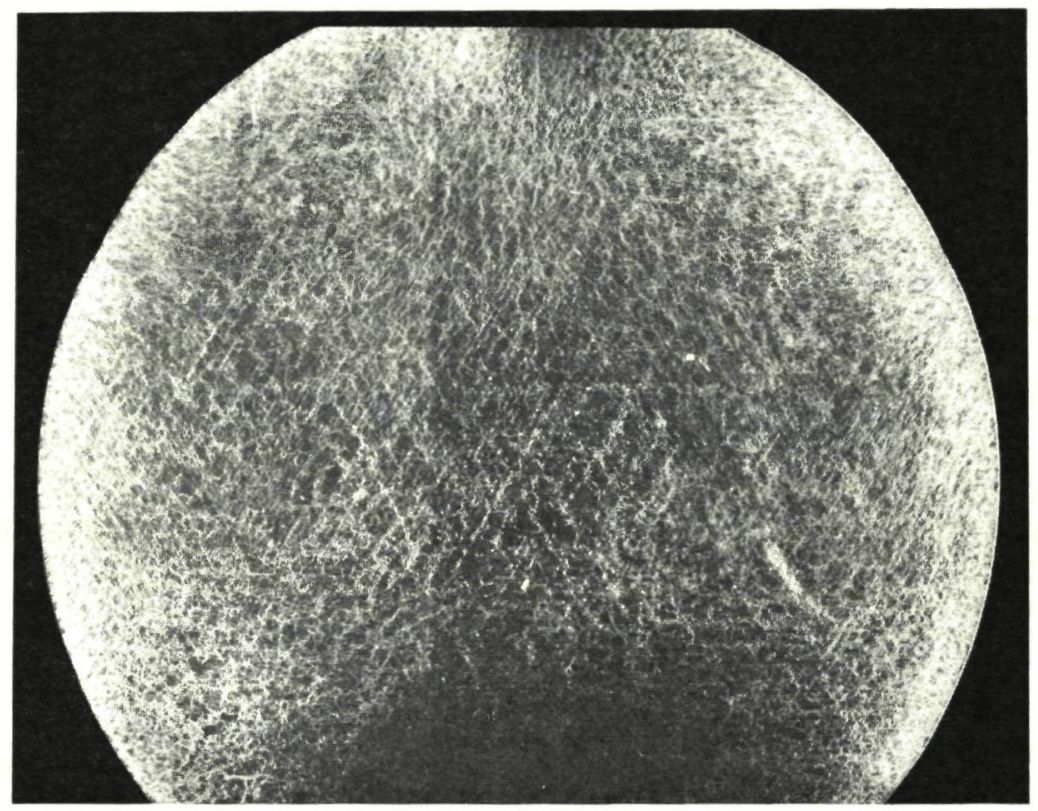

Fig. 2.5. (220) X-ray transmission topograph of a (111) cross-sectional silicon slice containing dislocations and impurity precipitates in the crystal centre. $\mathrm{MoK} \alpha_{1}$ radiation.

Microdefects, which are surrounded by strain fields smaller than about $10^{-4} \mathrm{~cm}$ across, have a small influence on X-ray diffraction ${ }^{2-35}$ ). The diffracted intensity, originating from these microdefects is buried in the background intensity diffracted by the perfect regions of the crystal. Consequently, these defects will remain undetected in normal Lang topography. However, the lattice distortion can be enlarged artificially by decorating the microdefects with copper and, as will be shown later in this study, also with lithium. The strain field around the decorated defects can be made sufficiently large to cause strong direct-image contrast. This combination of decoration and X-ray topography has been found to be of paramount importance for the study of vacancy clusters. 


\section{REFERENCES}

2-1) E. Sirtl and A. Adler, Z. Metallk. 52, 529, 1961.

2-2) T. Abe, T. Samızo and S. Maruyama, Jap. J. appl. Phys. 5, 458, 1966.

2-3) I. L. Shul'pına, L. V. Lainer, M. G. Milvidskii and E. P. Rashevskaya, Sov. Phys.-Solid State 9, $1291,1967$.

2-4) J. M. Hedges and J. W. Mitchell, Phil. Mag. 44, 223, 357, 1953.

2-5) S. A melinckx, Phil. Mag. 1, 269, 1956.

2-6) O. Deutschbein and M. Bernard, Symposium on Solid State Physics and Telecommunications (Brussels 1958), Academic Press Inc., New York, 1960.

2-7) W. C. Dash, J. appl. Phys. 27, 1193, 1956.

2-8) R. Bullough, R. C. Newman, J. Wakefıeld and J. B. Wıllıs, J. appl. Phys. 31, $707,1960$.

2-9) Y. Tokumaru, Jap. J. appl. Phys. 2, 542, 1963.

2-10) M. Yoshida and K. Furusho, Jap. J. appl. Phys. 3, 521, 1964.

2-11) M. Yoshida and K. Furusho, Jap. J. appl. Phys. 4, 74, 1965.

2-12) R. Bullough and R. C. Newman, Progress in semiconductors, Heywood, London, 1963, vol. 7, p. 101.

2-13) T. Takabatake, T. Furuya and Y. Ueda, Jap. J. appl. Phys. 9, 416, 1970.

2-14) W. C. Dash, J. appl. Phys. 29, 705, 1958.

2-15) W. C. Dash, Phys. Rev. Letters 1, 400, 1958.

2-16) W. C. Dash, J. appl. Phys. 30, 459, 1959.

2-17) G. H. Schwuttke, J. electrochem. Soc. 108, 163, 1961.

2-18) D. J. D. Thomas, Phys. Stat. sol. 3, 2261, 1963.

2-19) H. Peibst, Kristall und Technik 1, 51, 1966.

2-20) H. Rieger and K. Seiler, Z. Naturf. 16a, 220, 1961.

2-21) H. Rieger, Phys. Stat. sol. 7, 685, 1964.

2-22) L. Fiermans and J. Vennik, Phys. Stat. sol. 12, 277, 1965.

2-23) L. Fiermans and J. Vennik, Phys. Stat. sol. 21, 627, 1967.

2-24) L. Fiermans and J. Vennik, Phys. Stat. sol. 22, 463, 1967.

2-25) F. A. Trumbore, Bell Sys. tech. J. 39, 205, 1960.

2-26) E. M. Pell, Phys. Rev. 119, 1222, 1960.

2-27) E. M. Pell, Symposium on Solıd State Physics and Telecommunications (Brussels 1958), Academic Press Inc., New York, 1960, vol. 1, p. 261.

2-28) R. D. Weltzin, Act. met. 13, 115, 1965.

2-29) J. W. Ferman and R. Swalin, in De Boer (ed.), Reacuvity of solids, Elsevier Publishing Company, Inc., Houston, 1962, p. 264.

2-30) J. W. Ferman, J. appl. Phys. 39, 3771, 1968.

2-31) R. C. Young, J. W. Westhead and J. C. Corelli, J. appl. Phys. 40, 271, 1969.

2-32) L. B. Valdes, Proc. IRE 42, 420, 1954.

2-33) A. R. Lang, J. appl. Phys. 30, 1748, 1959.

2-34) B. Batterman and H. Cole, Rev. mod. Phys. 36, 681, 1964.

2-35) J. Chikawa, Y. Asaeda and 1. Fujimoto, J. appl. Phys. 41, 1922, 1970. 


\section{EXPERIMENTAL RESULTS}

\subsection{Introduction}

A number of silicon crystals were grown by means of the pedestal-pulling method (sec. 1.2.2). The crystal-growth process took place in a water-cooled metal vessel filled with purified argon of atmospheric pressure. The argon was purified by means of heated copper and molecular sieves. Highly resistive polycrystalline silicon rods were used as starting material. Dislocations were eliminated by application of the Dash method (sec. 1.2.3). Most crystals were not intentionally doped $(\varrho \approx 1000 \Omega \mathrm{cm}$ ) but in some cases phosphorus was added during growth in order to obtain electrical resistivities of about 5 to $10 \Omega \mathrm{cm}$. The crystals were grown either in the $\langle 111\rangle$ or $\langle 100\rangle$ direction, and their diameters amounted to 21 to $28 \mathrm{~mm}$.

The oxygen concentration in these crystals was lower than $5.10^{15} \mathrm{~cm}^{-3}$ as was established by infrared-absorption spectroscopy. The carbon content is not known, but is probably low because the starting material was subjected to zone-refining treatments prior to growth. Metallic impurities were not detected by mass spectroscopy indicating their concentration to be a few p.p.b. or less.

The perfection of these crystals, particularly in relation to the occurrence of vacancy clusters, has been studied using the techniques described in chapter 2 .

Apart from these crystals a variety of dislocation-free floating-zone crystals with different levels of doping, obtained from various silicon manufacturers, has been investigated. The exact growth conditions of these crystals were generally not known. The results of these investigations are presented in this chapter.

The fabrication of micro-electronic devices made on silicon material requires high-temperature treatments in oxidizing or inert atmospheres. The influence of such heat treatments on the distribution and shape of the vacancy clusters as well as the influence of the clusters on the performance of planar devices has also been examined.

\subsection{Results obtained with preferential etching}

Both longitudinal and cross-sectional slices were cut from the crystals to be investigated. After chemically-mechanically polishing, these slices were subjected for several minutes to the preferential etchant consisting of a mixture of $\mathrm{HF}, \mathrm{H}_{2} \mathrm{O}$ and $\mathrm{CrO}_{3}$ (sec. 2.2). The distribution of the etch pits formed after this treatment was studied with an optical microscope. Figure 3.1 shows the etch-pit distribution, as revealed on a longitudinal crystal section, which was typical for all crystals investigated. The etch pits are concentrated in narrow curved bands, which are separated from each other by regions with a very low 
a)
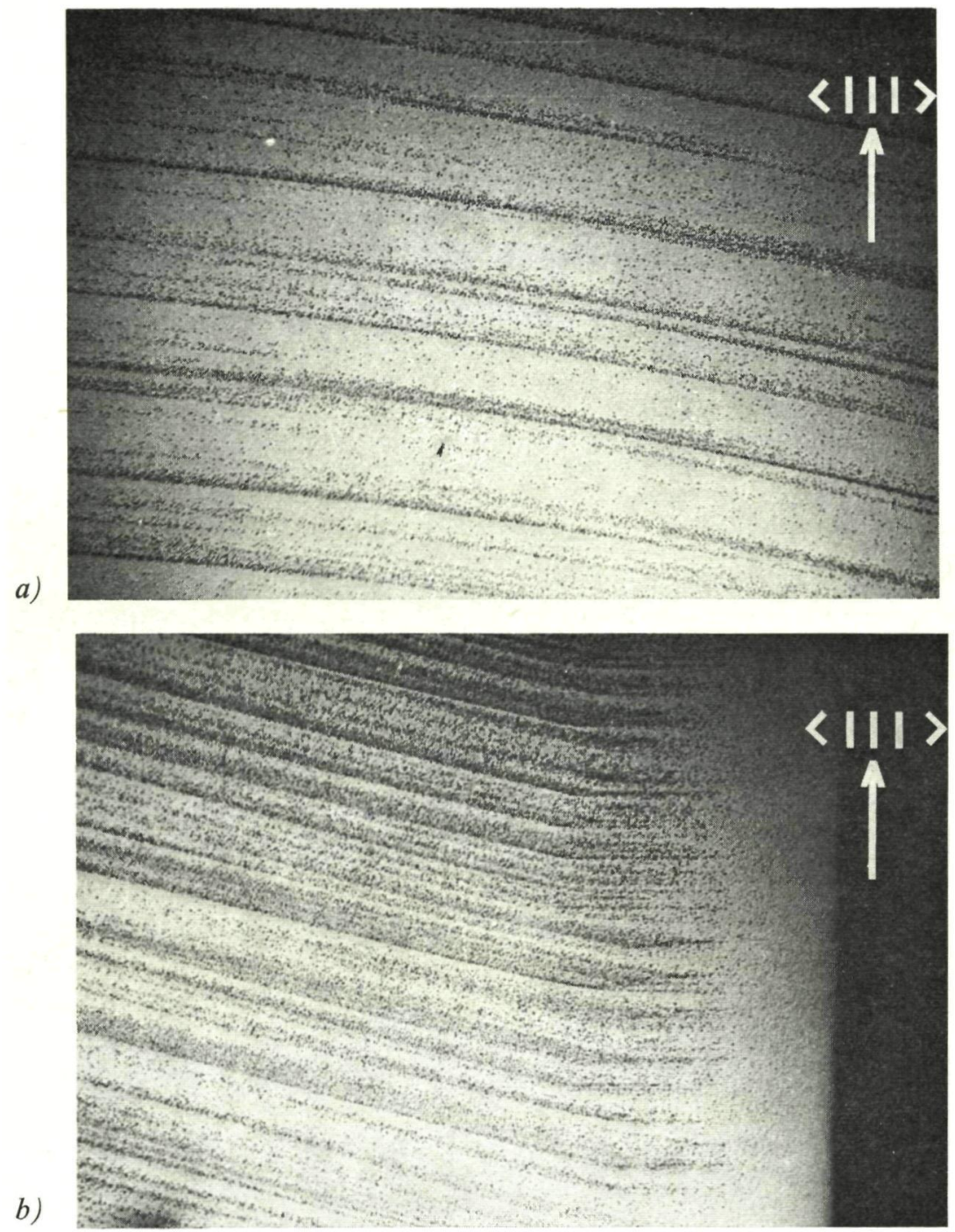

Fig. 3.1. Preferentially etched longitudinal section of a dislocation-free floating-zone silicon crystal grown in the $\langle 111\rangle$ direction. Magnification: $20 \times$.

(a) Central region of the crystal. (b) Edge region of the crystal.

density of the etch pits. In most crystals these bands exhibit randomly distributed interruptions.

During etching new pits are continuously formed. This, in combination with the small size of the pits, indicates that the etch pits are caused by small local defects present in the silicon lattice. In floating-zone crystals containing dislocations no such etch pits are formed, which means that the microdefects giving rise to their formation are not present in dislocated crystals. 
From fig. 3.1 it can be concluded that the concentration of the microdefects exhibits a minimum in the central region of the crystal. Furthermore, no etch pits are revealed in a region about $2 \mathrm{~mm}$ in width, adjacent to the crystal surface (fig. 3.1(b)).

Preferential etching of cross-sectional surfaces reveals an etch-pit pattern in the form of an interrupted spiral (fig. 3.2). Again a pronounced minimum in

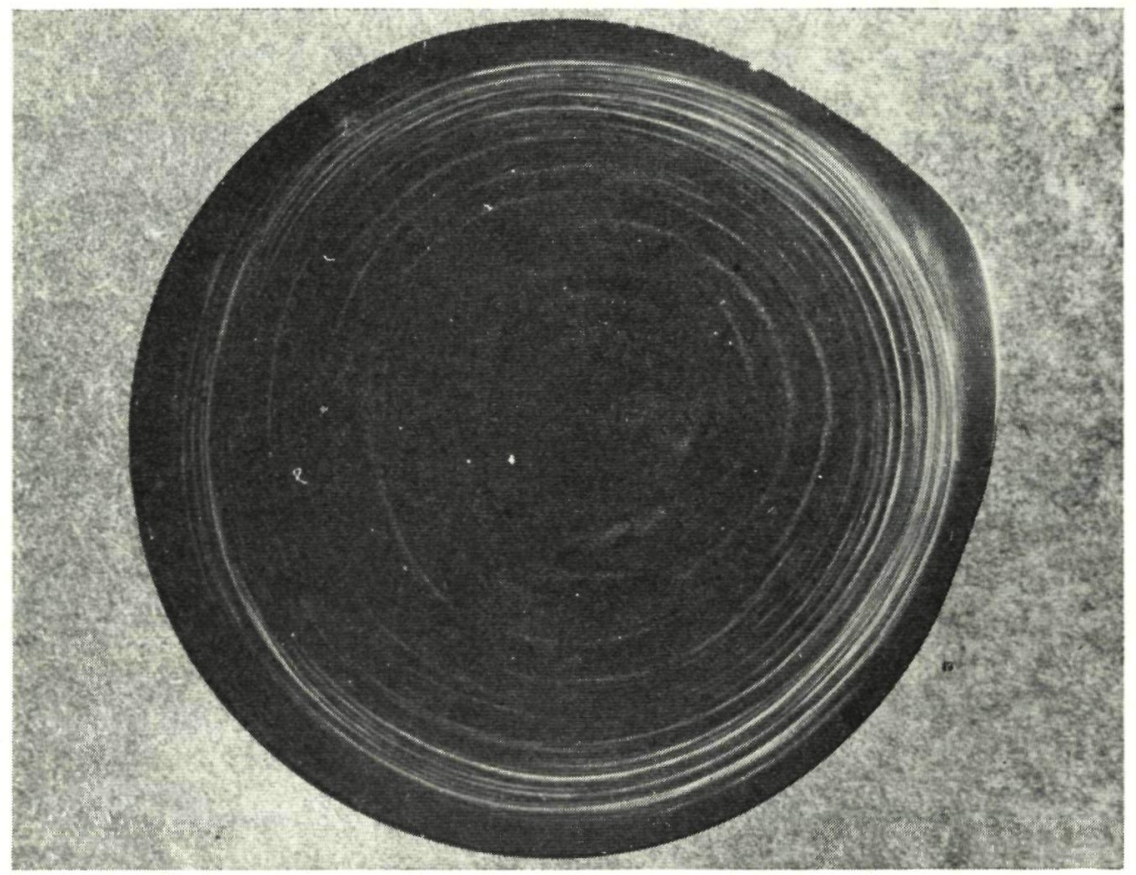

Fig. 3.2. Preferentially etched cross-sectional surface of a dislocation-free crystal grown in the $\langle 111\rangle$ direction by the pedestal-pulling method, in argon. Growth rate: $3 \mathrm{~mm} / \mathrm{min}$. Crystal diameter: $22 \mathrm{~mm}$.

the microdefect concentration in the crystal centre can be noticed. On such (111) planes the etch pits are generally more or less triangular in shape with a flat bottom (fig. 3.3). The three edges of the pits are parallel to the $\langle\overline{1} 10\rangle$ directions. However, besides triangular pits, irregularly shaped pits have sometimes been observed. If the sample surface is of an arbitrary orientation, the shape of the pits is often less clearly related to the crystallographic directions. On the longitudinal surface shown in fig. 3.1( $a$ ), for instance, the pits have a circular shape.

Superimposed on the above-mentioned distribution of etch pits another distribution of very tiny etch pits is sometimes observed in the optical microscope. This indicates that a second group of microdefects can be present in the asgrown crystals. Figure 3.4 shows both types of etch pits as revealed on a care- 


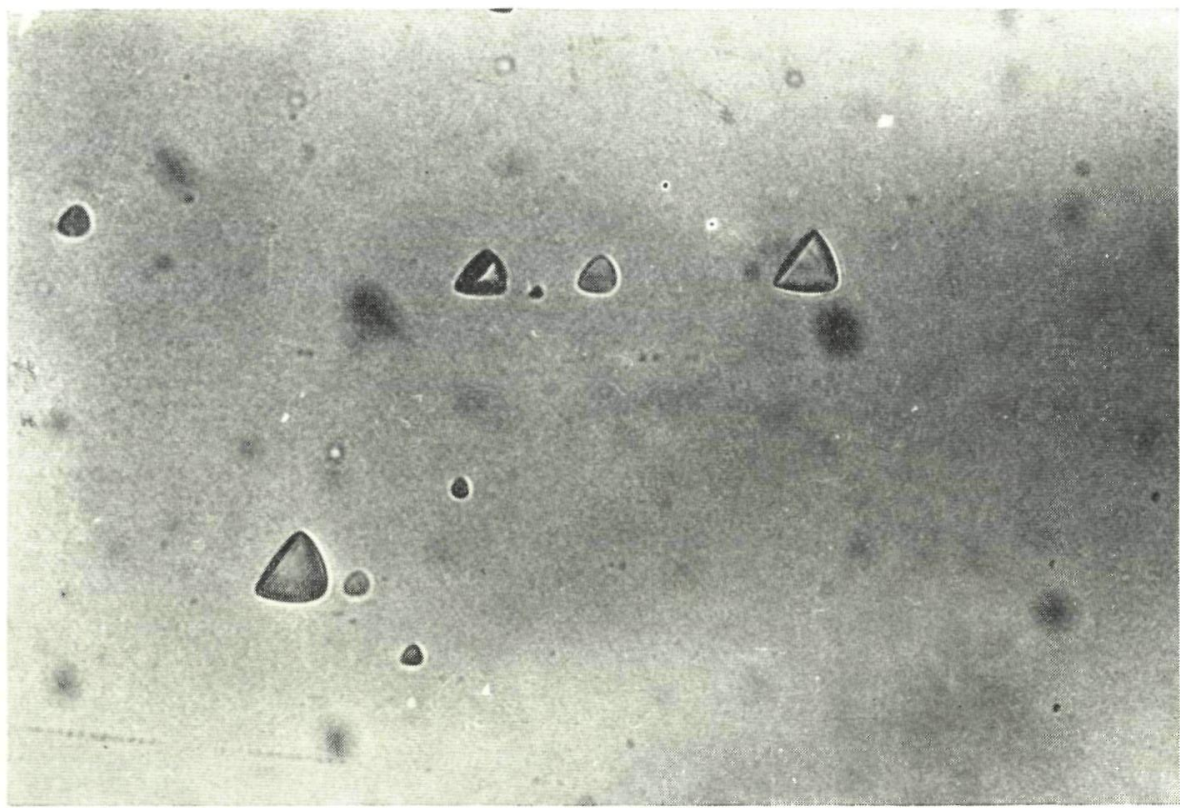

Fig. 3.3. Flat-bottomed pits revealed by preferential etching on a (111) cross-sectional surface. Magnification: $500 \times$.

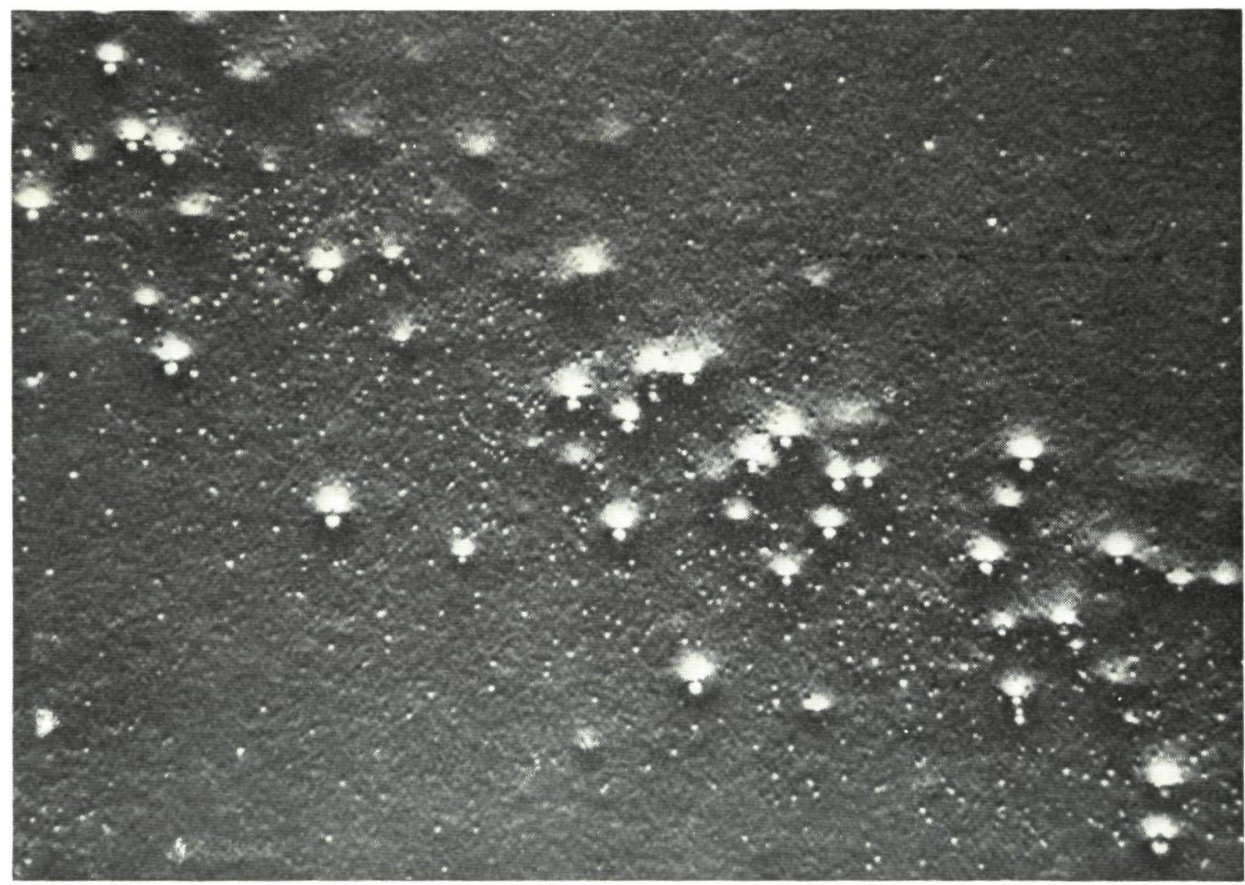

Fig. 3.4. Microscopic detail of the sample shown in fig. 3.2. Two types of etch pits, differing in size, are revealed. Nomarski interference contrast. Magnification $230 \times$. 
fully polished cross-section of a crystal grown in argon. The number of tiny pits per $\mathrm{mm}^{2}$ is about 15 times as high as that of the la.ger ones. Both types of pits are found in the bulk of the crystal, whereas the small pits are found only in the central region of the crystal cone and in the crystal surface region. The size of the tiny pits is maximum in those regions where the larger ones are absent. In most crystals, however, the distribution of the second type of pit is not observed in the optical microscope. The possibility exists that these pits, although present, remain undetected in the optical microscope because of the limited resolution of this instrument. In order to examine this, shadowed carbon replicas were made of briefly preferentially etched polished surfaces similar to those shown in fig. 3.1. These replicas were then investigated in the transmission electron microscope. Although continuously new etch pits are formed and their size increases with increasing etching time, two classes of etch pits were distinguished. Apart from the relatively large pits, numerous tiny pits are formed (fig. 3.5). These pits, which are about 0.05 to $0.1 \mu \mathrm{m}$ across, were absent in spherical regions around the large ones. In replicas made of regions between the defect striations, where the concentration of the large pits was nearly zero, only a few small ones could be detected. This indicated that the small defects were present in the same striated distribution as that of the larger ones.

It is well known ${ }^{3-1}$ ) that preferential etching also reveals the dope-striation pattern if the level of doping is sufficiently high (sec. 1.3.2). The variation in dope concentration causes a variation in etching rate. These striations reflect the shape of the solid-liquid interface present during growth. By applying the etch treatment on samples taken from a doped dislocation-free crystal containing $6.10^{16}$ phosphorus atoms $/ \mathrm{cm}^{3}$, the microdefect distribution together with the dope striations could be observed. It was found that the microdefects were grouped in regions located at one side along the dope striations as is illustrated in fig. 3.6. Consequently, the microdefect distribution in the bulk of the crystal has the form of an interrupted spiral ramp. The pitch of the spiral ramp is equal to $V_{g} / R^{\prime}$, where $V_{g}$ is the crystal-growth rate and $R^{\prime}$ the crystal-rotation rate. Furthermore, these results indicate that the periodic variation in growth rate usually occurring plays an important part in the formation process of the defects.

\subsection{Results obtained with copper decoration}

If X-ray topographs are made of slices $\left(t^{\prime} \approx 250 \mu \mathrm{m}\right)$ taken from floatingzone dislocation-free crystals containing microdefects, no contrast is observed. This indicates that the lattice distortion around these microdefects is too small to cause an observable change in the intensity of the diffracted X-ray beam. However, if the defects are decorated with copper the lattice distortion is enlarged to such an extent that a strong contrast becomes visible. 


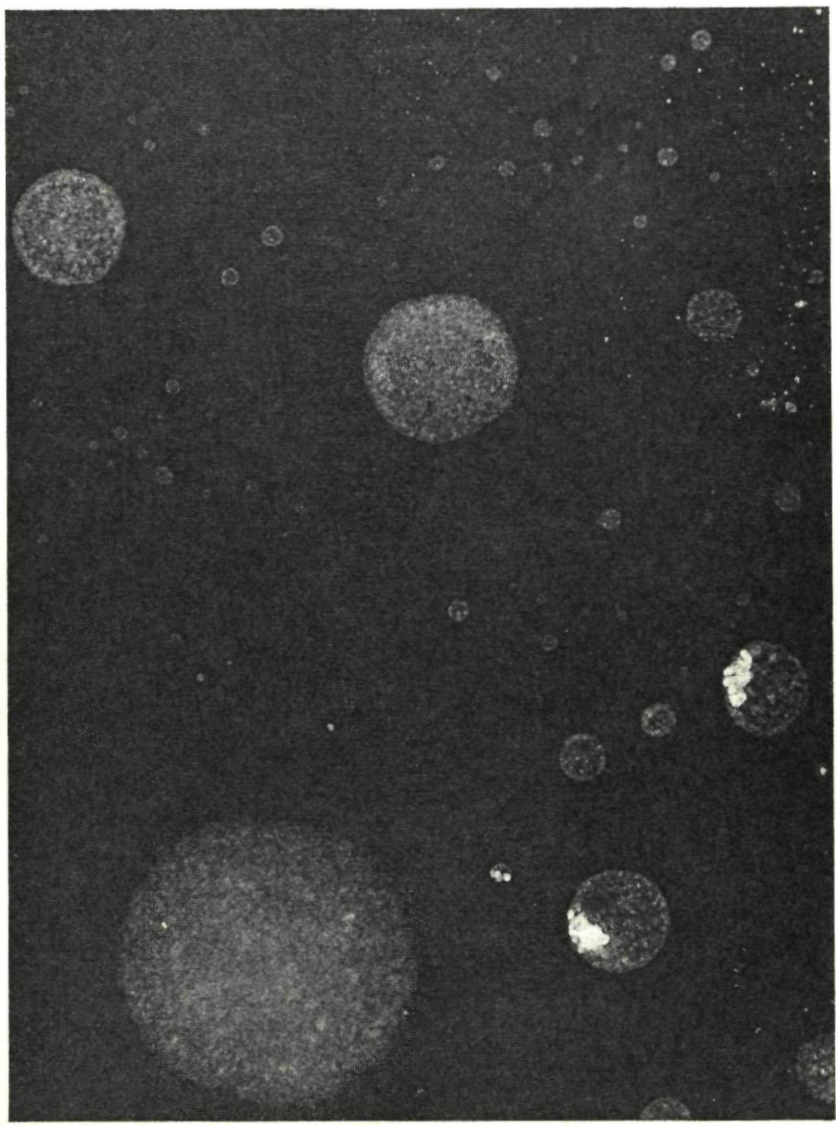

Fig. 3.5. Transmission electronmicroscopic photograph of a carbon replica made of a preferentially etched longitudinal section of a dislocation-free silicon crystal. Diameter of largest etch pit is $10^{-4} \mathrm{~cm}$.

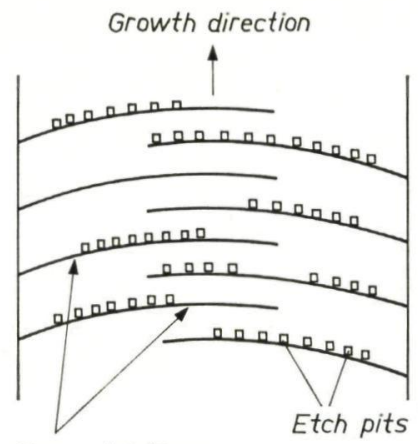

Dope striations

Fig. 3.6. Schematical plot of the etch-pit distribution and dope striations as revealed by preferential etching of a longitudinal section taken from a doped, dislocation-free F.Z. silicon crystal. The etch pits are located on one side along the dope striations. 
The required copper decorations were carried out following the procedure described in sec. 2.3. A diffusion temperature of $950^{\circ} \mathrm{C}$ was chosen. At this temperature the amount of copper introduced was sufficient for a strong defect decoration, while plastic deformation of the samples could be prevented in most of the experiments.

In a dislocation-free crystal four regions (I-IV), which are grown under different conditions, can be distinguished. The first one is the thin neck (I) adjacent to the seed, where the dislocations are eliminated (sec. 1.2.3). This region is successively followed by the cone (II), where the diameter is increased to its required value by reducing the h.f. power, the main part of the crystal (III) grown with a more or less constant diameter and finally the region IV, where the growth procedure is terminated by gradually increasing the h.f. power until the crystal diameter is reduced to zero.

Longitudinal as well as cross-sectional slices were cut from the regions I to IV of the various crystals and subjected to the copper-decoration treatment. Although the method of diffusion prevents surface contamination during the decoration (sec. 2.3) some precipitation of copper at the surface was detected in those crystal regions where the density of microdefects was very low (e.g. in between the striations). In those regions the supersaturated amount of copper partly diffuses out to the sample surface during cooling, because bulk precipitation is almost absent. In order to prevent image formation of these surface precipitates on the X-ray topographs a thin surface layer was removed from both sides of the decorated slices by non-preferential etching. The X-ray transmission topography was carried out with the aid of a Lang-type Jarrell Ash camera. The topographs were made with $\mathrm{MoK} \alpha_{1}$ radiation on Ilford L4 nuclear plates with an emulsion thickness of $50 \mu \mathrm{m}$. The thickness of the samples was in the range of 200 to $300 \mu \mathrm{m}$, which means that the condition for directimage-contrast formation $\left(\mu t^{\prime}<1\right)$ was fulfilled. Consequently, lattice defects will cause black (positive) contrast on the nuclear plates. However, except for those photographs showing microscopic details, the contrast on the pictures has been reversed when compared with that on the original plates.

Figure 3.7 shows $\mathrm{X}$-ray transmission topographs of copper-decorated longitudinal sections taken from the regions II (fig. 3.7(a)), III (fig. 3.7(b)) and IV (fig. 3.7(c)) of an undoped crystal grown by the pedestal-pulling method in purified argon of $1 \mathrm{~atm}$. The growth axis is oriented in the $\langle 111\rangle$ direction. The growth rate amounted to $1 \mathrm{~mm} / \mathrm{min}$ in the regions II and III, but was increased to $2 \mathrm{~mm} / \mathrm{min}$ in the area shown in fig. 3.7(c). The decorated microdefects are visible as white dots. Apart from a few exceptions (e.g. fig. 3.7(c)) no dislocations are introduced during the decoration procedure. In figs $3.8(a)$ and 3.9(a) details are shown of the crystal regions marked $\mathrm{E}$ and $\mathrm{B}$ in fig. 3.7(b). Infrared transmission micrographs of the same areas are shown in figs $3.8(b)$ and $3.9(b)$. 


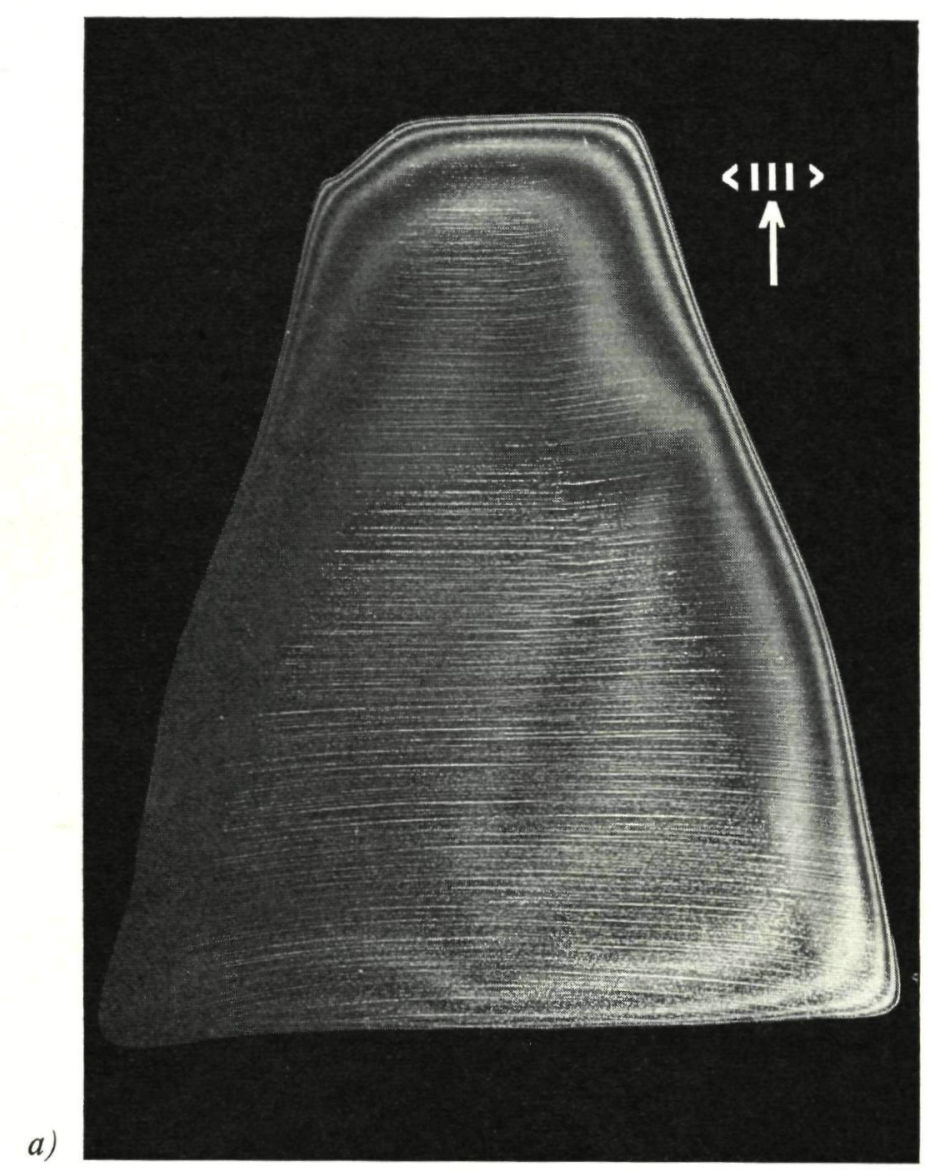

Fig. 3.7. X-ray transmission topographs of copper-decorated longitudinal sections taken from a pedestal-pulled crystal, grown in argon. Crystal diameter: $21 \mathrm{~mm}$. Reflecting planes: (111). (a) Cone region (II); growth rate: $1 \mathrm{~mm} / \mathrm{min}$. 


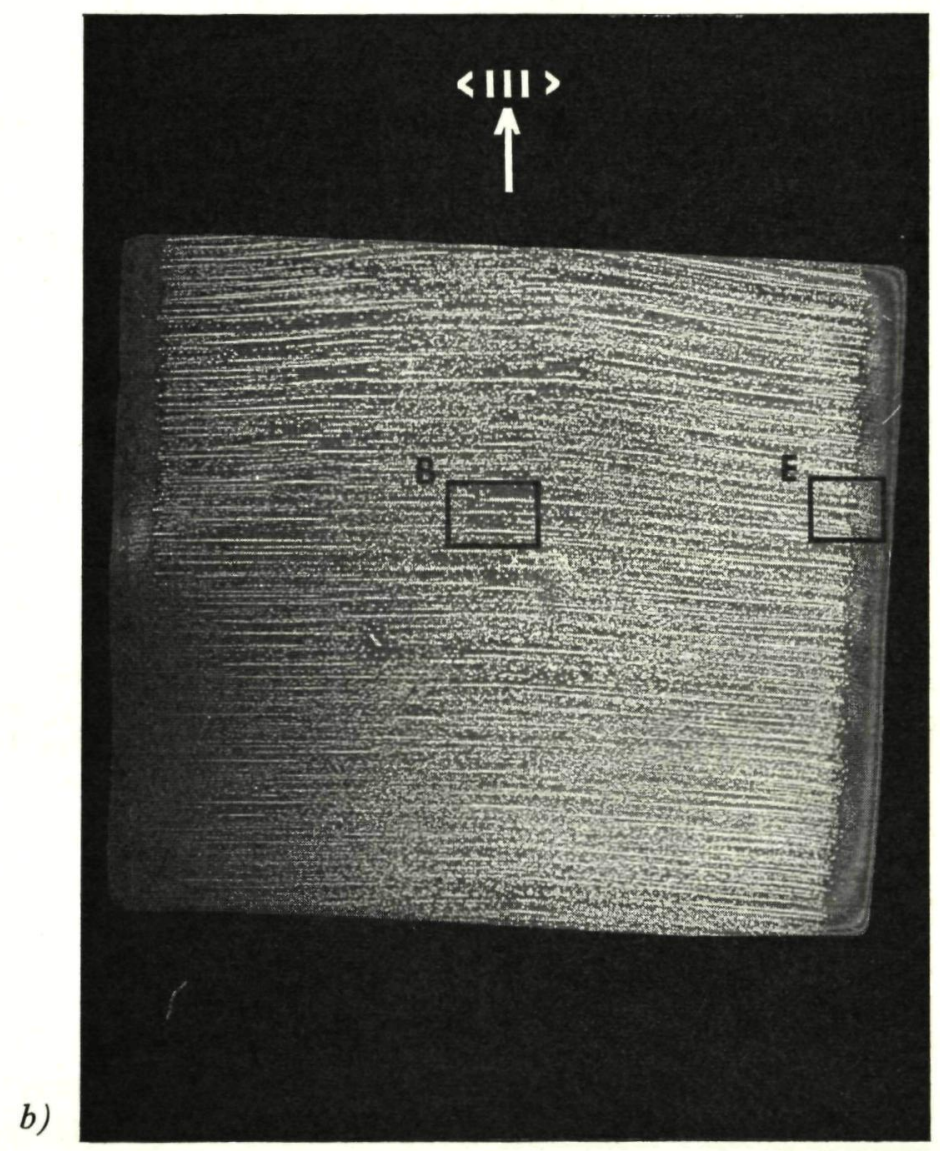

Fig. 3.7. (b) Central region (III); growth rate: $1 \mathrm{~mm} / \mathrm{min}$; concentration of A-clusters: $1 \cdot 1 \cdot 10^{6} \mathrm{~cm}^{-3}$. 


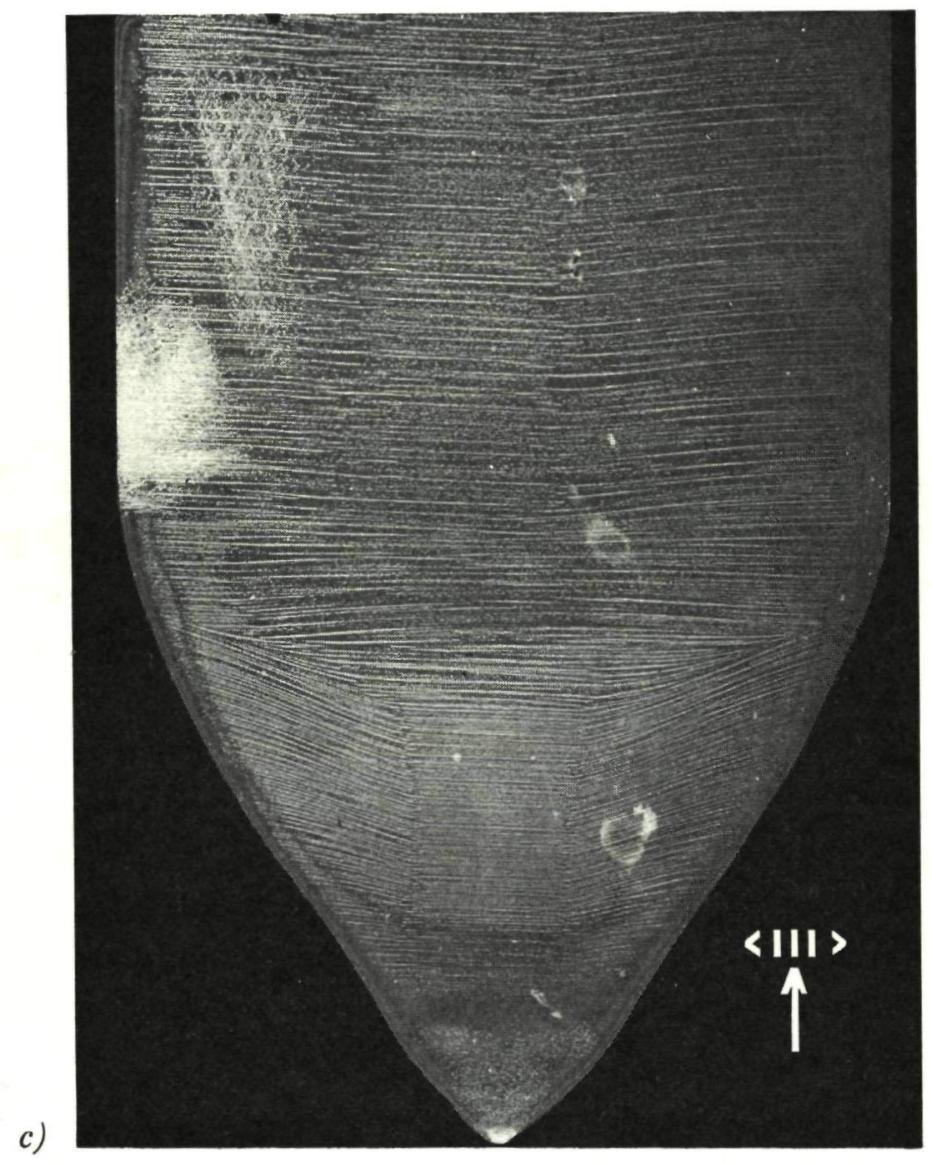

Fig. 3.7. (c) Lower region (IV); growth rate: $2 \mathrm{~mm} / \mathrm{min}$; dislocations are introduced during the decoration procedure. 
a)
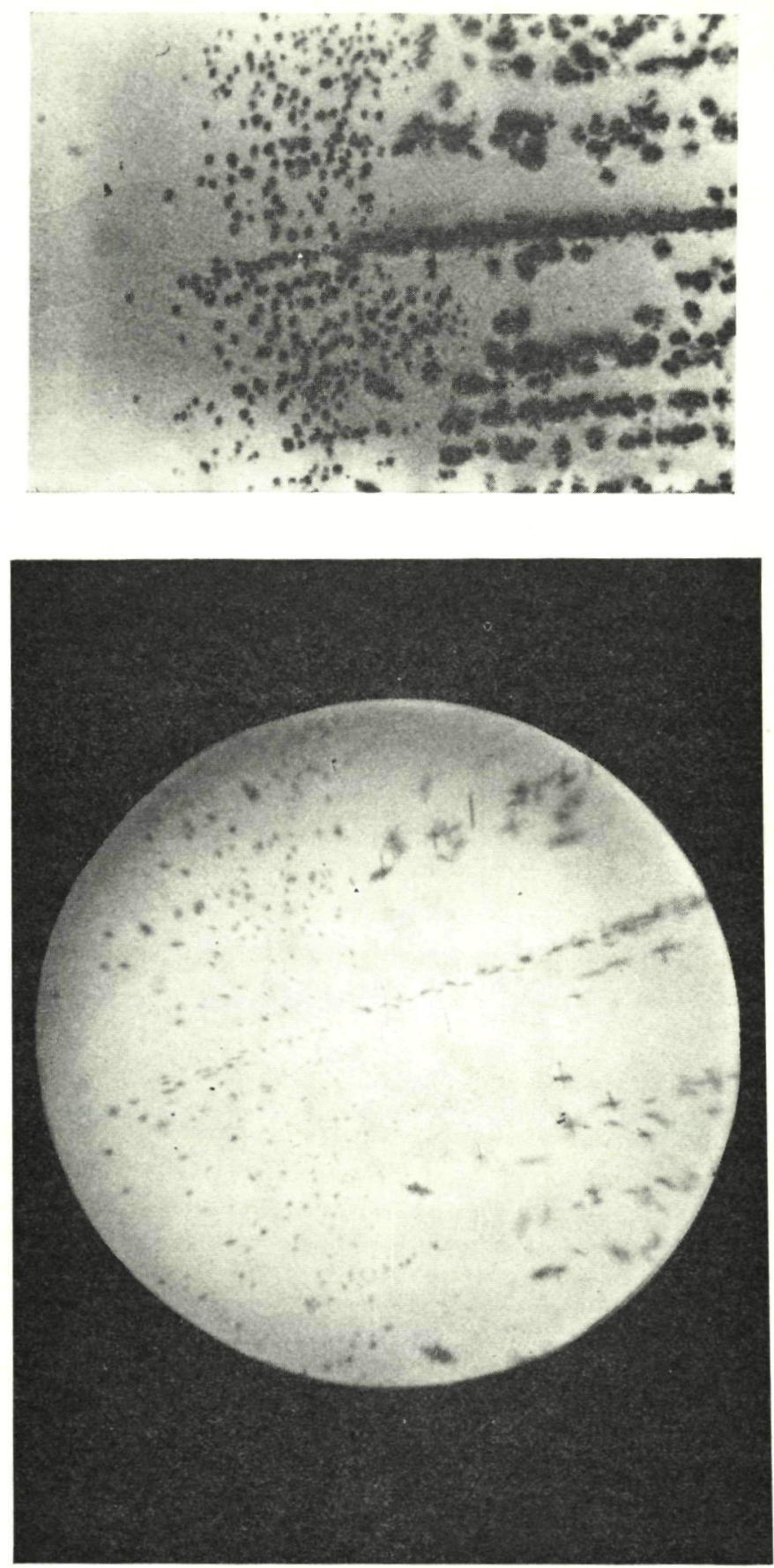

Fig. 3.8. (a) Detail of the edge at $\mathrm{E}$ of the crystal shown in fig. 3.7(b); X-ray topograph revealing A- and B-clusters. (b) Infrared transmission micrograph of the same region. 

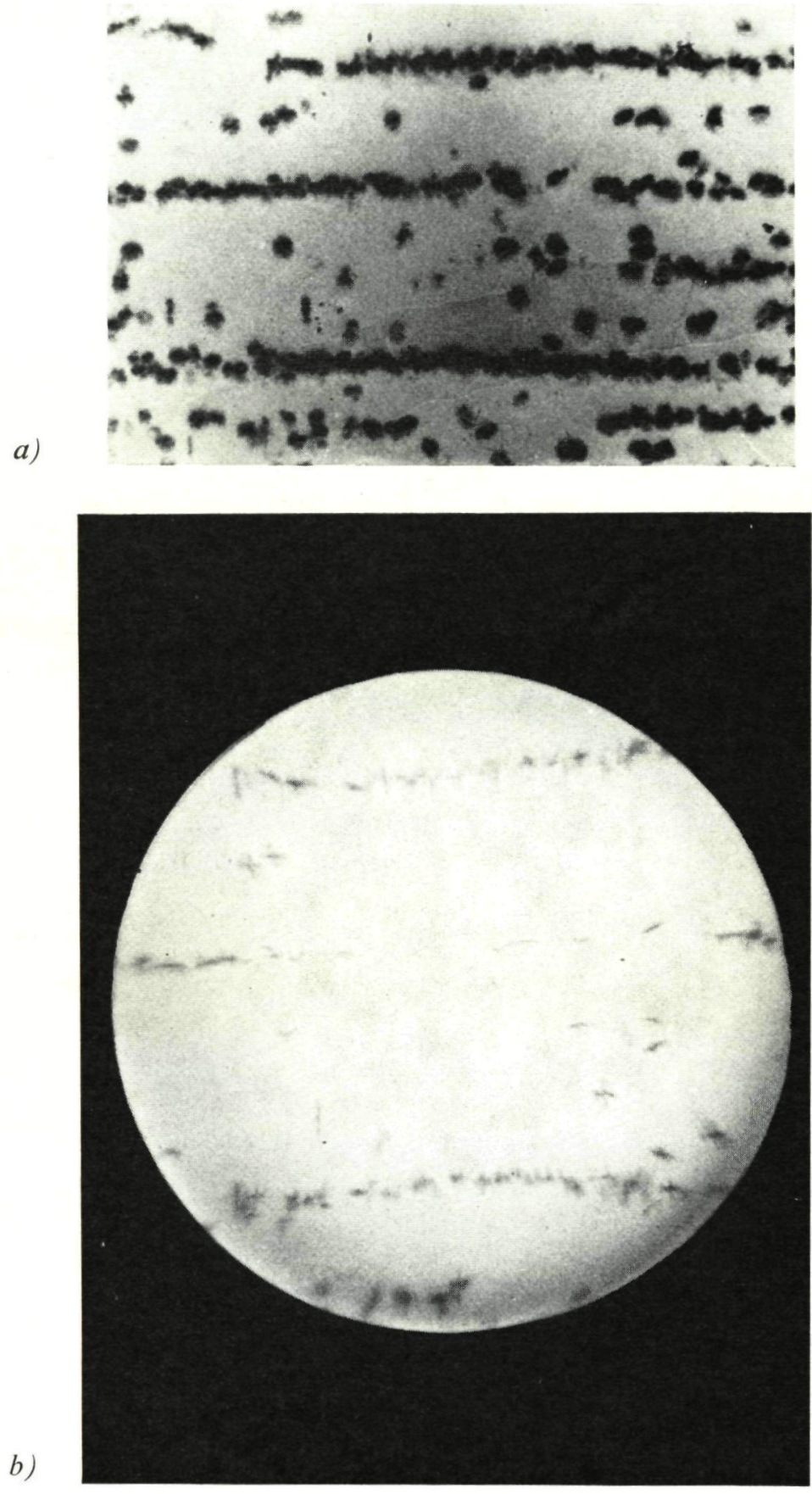

Fig. 3.9. (a) Detail of the bulk region at B of the crystal shown in fig. 3.7(b); X-ray topograph revealing A-clusters. (b) Infrared transmission micrograph of the same region. 
The defect distribution as present in a cross-sectional slice, cut from a similar crystal grown in argon at $1 \mathrm{~mm} / \mathrm{min}$, is shown in fig. 3.10 .

Figures 3.11 and $3.12 *$ ) show the microdefect configurations in crystals grown in argon at a rate of $3 \mathrm{~mm} / \mathrm{min}$ in the $\langle 111\rangle$ and $\langle 100\rangle$ directions respectively. Topographic and infrared micrographic details of the edge area at E in fig. 3.12(a) are shown in figs 3.13(a) and (b).

In crystals grown in vacuum, which were obtained from different suppliers, similar defect distributions were found (figs 3.14 and 3.15).

Sometimes the circular symmetry in the defect distribution is found to be heavily disturbed (fig. 3.16).

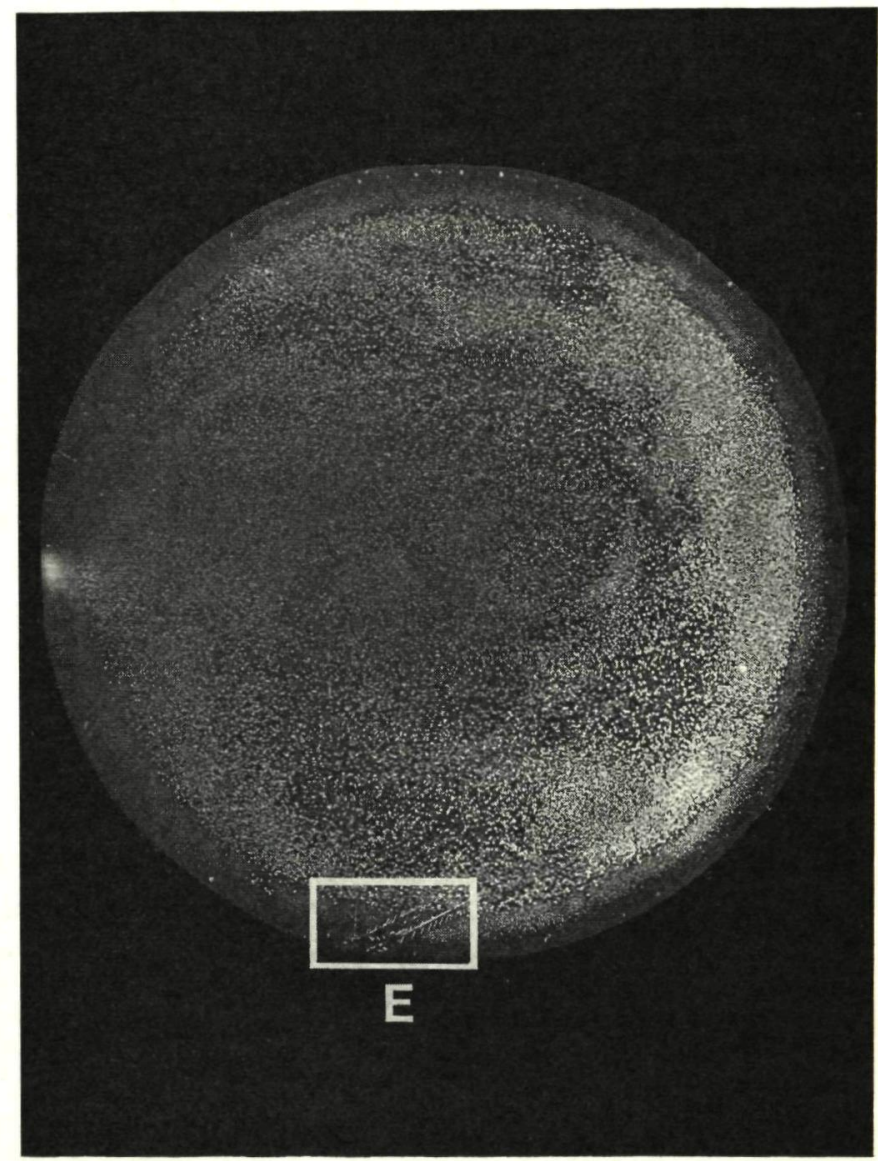

Fig. 3.10. X-ray transmission topograph of a copper-decorated cross-section from a pedestalpulled crystal grown in argon at $1 \mathrm{~mm} / \mathrm{min}$. Growth direction: $\langle 111\rangle ;(2 \overline{2} 0)$ reflex; crystal diameter: $21 \mathrm{~mm}$; concentration of A-clusters: $9 \cdot 10^{5} \mathrm{~cm}^{-3}$.

*) The vertical line pattern, often visible in the perfect crystal regions, is due to a periodic variation in the speed of the traverse movement of the crystal on the Lang camera during exposure. 


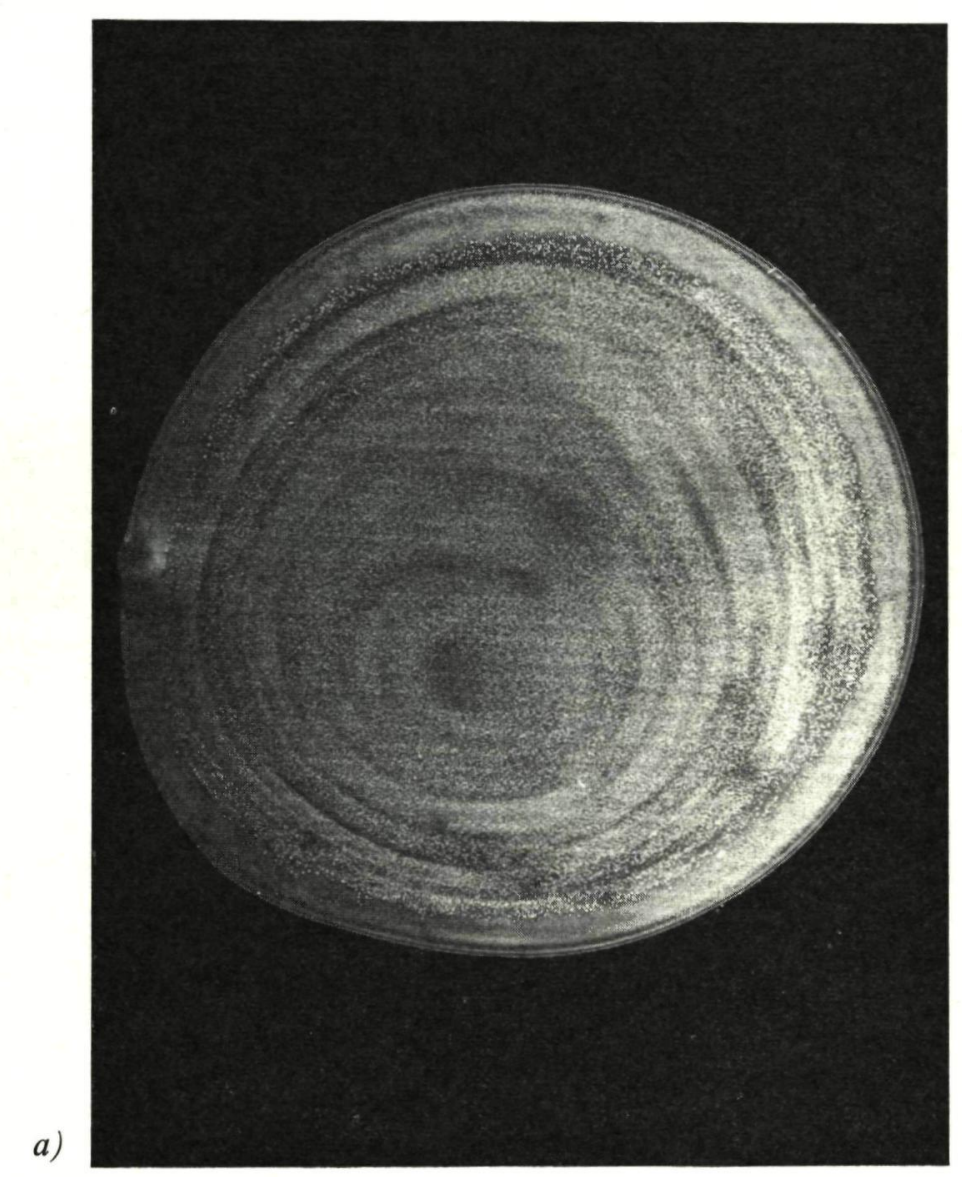

Fig. 3.11. X-ray transmission topographs of copper-decorated sections taken from a pedestalpulled crystal grown in argon at $3 \mathrm{~mm} / \mathrm{min}$. Growth direction $\langle 111\rangle$. (a) Cross-section taken from the same crystal as the slice shown in fig. 3.2: (220) reflex; concentration of A-clusters: $1 \cdot 6 \cdot 10^{6} \mathrm{~cm}^{-3}$. 


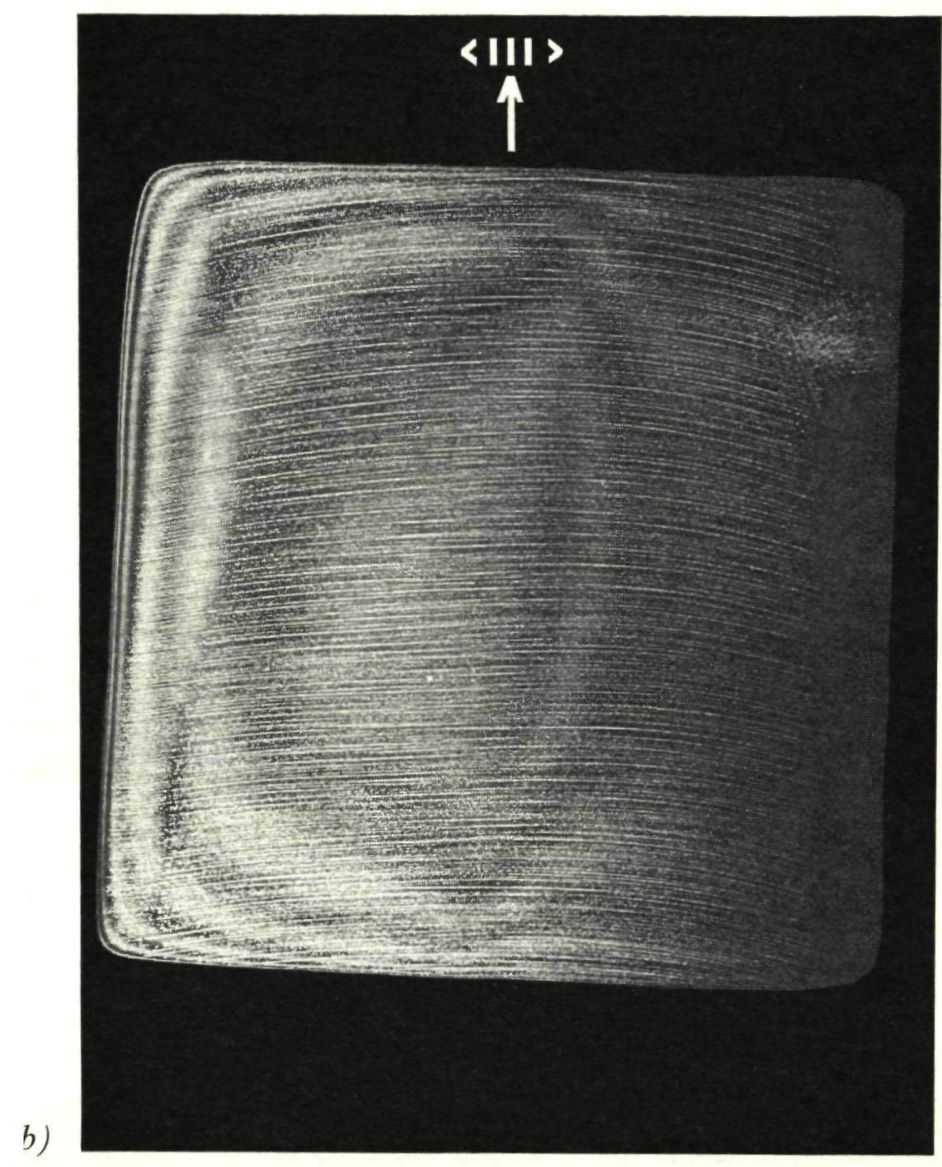

Fig. 3.11. (b) Corresponding longitudinal section: (111) reflex. 

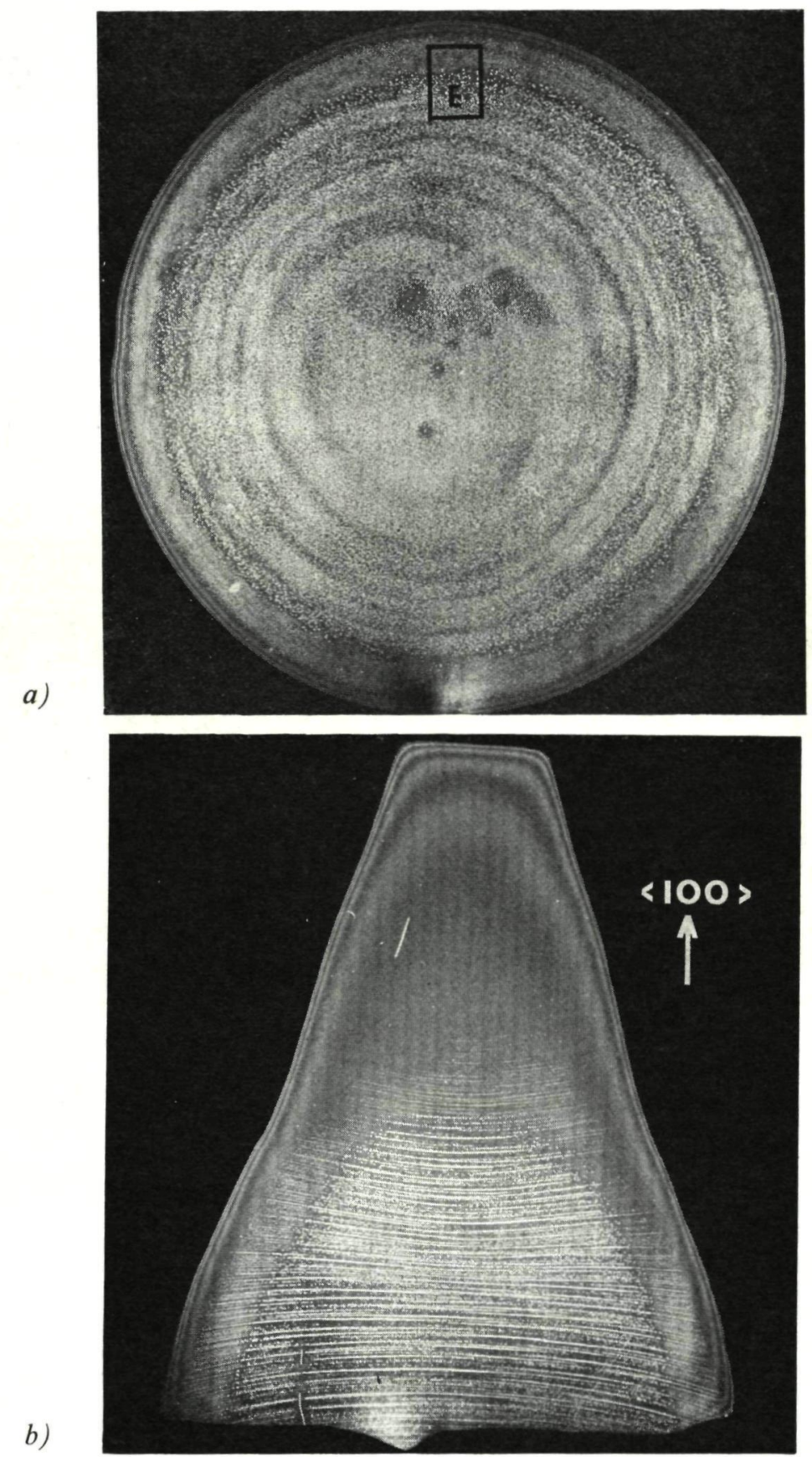

Fig. 3.12. X-ray transmission topographs of copper-decorated sections taken from a pedestalpulled crystal grown in argon at $3 \mathrm{~mm} / \mathrm{min}$. Growth direction: $\langle 100\rangle$. (a) Cross-section: $(2 \overline{2} 0)$ reflex; concentration of A-clusters: $2 \cdot 2 \cdot 10^{6} \mathrm{~cm}^{-3}$. (b) Longitudinal section of the cone region (II); (220) reflex. 
a)
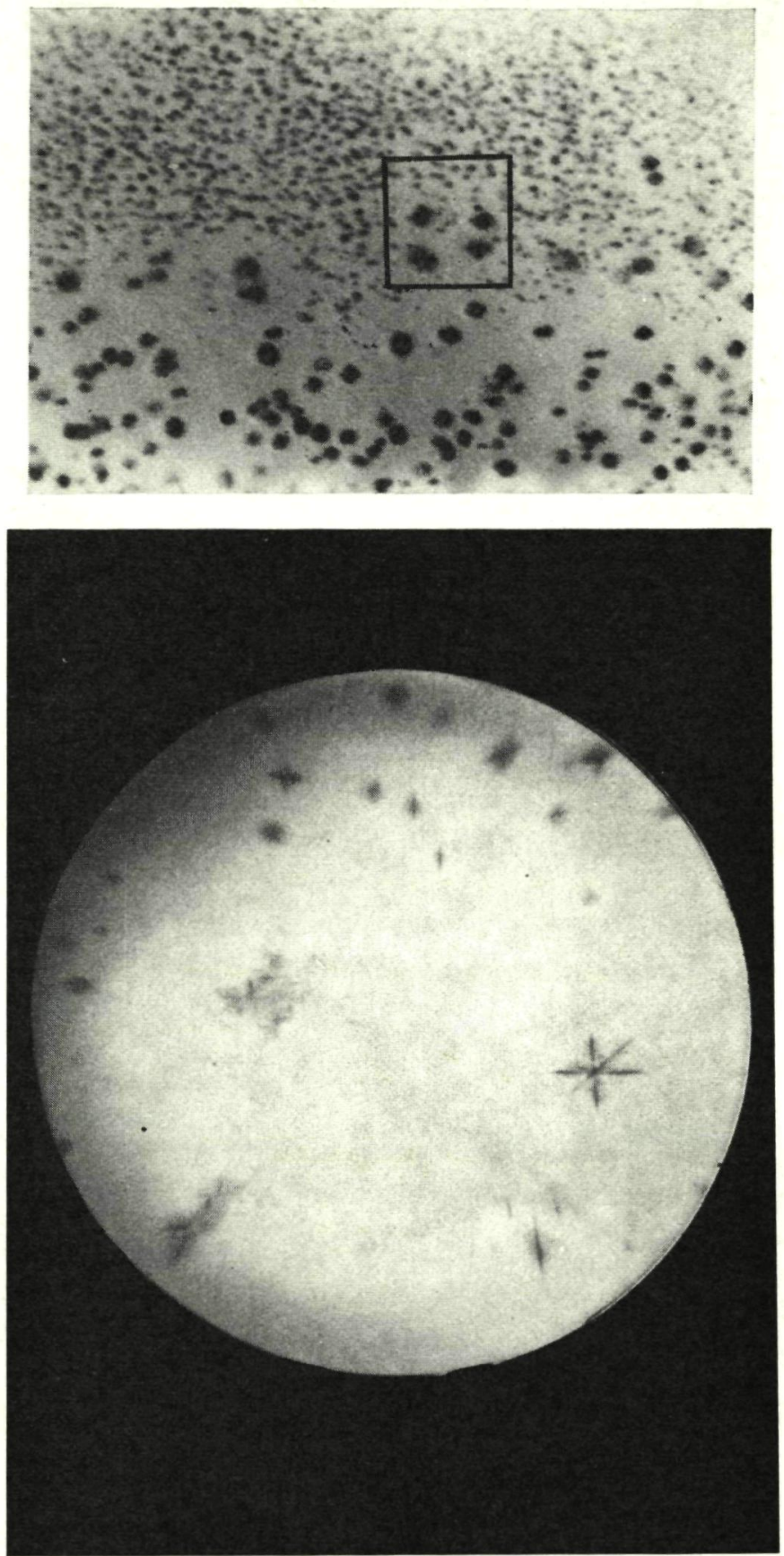

b)

Fig. 3.13. (a) Detail of the topograph in fig. 3.12(a) at E (edge region); A- and B-clusters are visible. (b) Infrared transmission micrograph of the central area of fig. 3.13(a) (see frame); the copper precipitates exhibit a dendritic fine structure. 


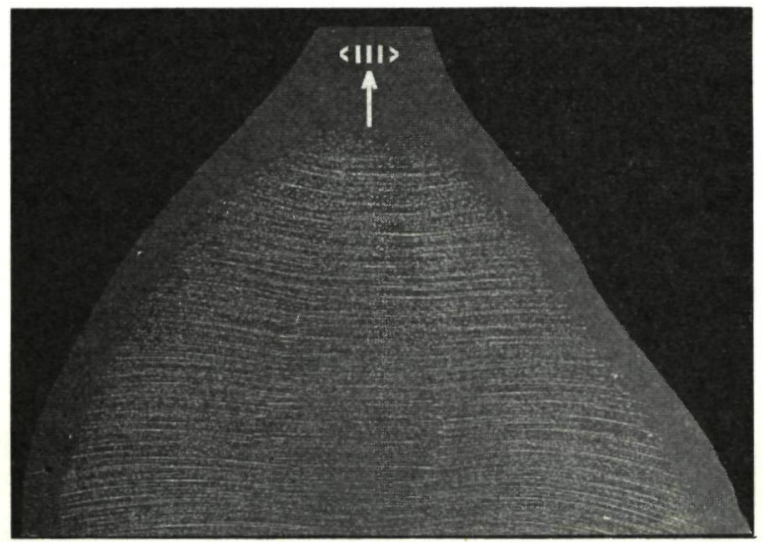

Fig. 3.14. X-ray transmission topograph of a copper-decorated longitudinal section of a crystal grown in high vacuum. Growth rate: $1.8 \mathrm{~mm} / \mathrm{min}$; growth direction: $\langle 111\rangle$; crystal diameter: $33 \mathrm{~mm}$; $(2 \overline{2} 0)$ reflex. No defects are observed near the crystal surface. Concentration of A-clusters: $7 \cdot 2 \cdot 10^{5} \mathrm{~cm}^{-3}$.

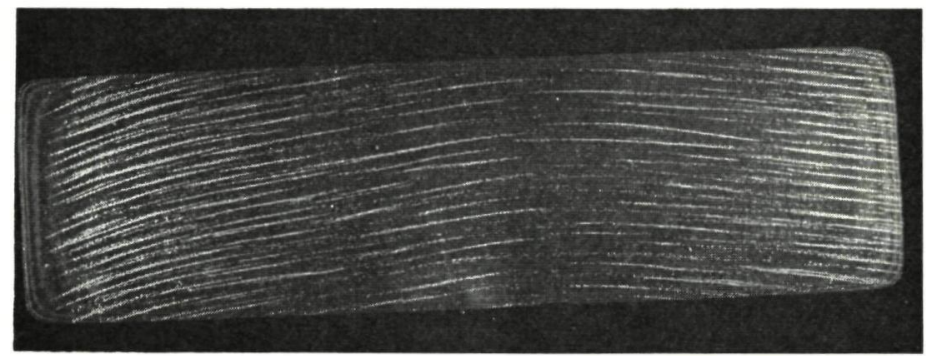

Fig. 3.15. X-ray transmission topograph of a copper-decorated longitudinal section of a crystal grown in high vacuum. Growth direction: $\langle 111\rangle$; crystal diameter: $41 \mathrm{~mm}$; (22̄0) reflex. Growth rate is unknown, but $V_{g} / R^{\prime}=0.5 \mathrm{~mm}$. 


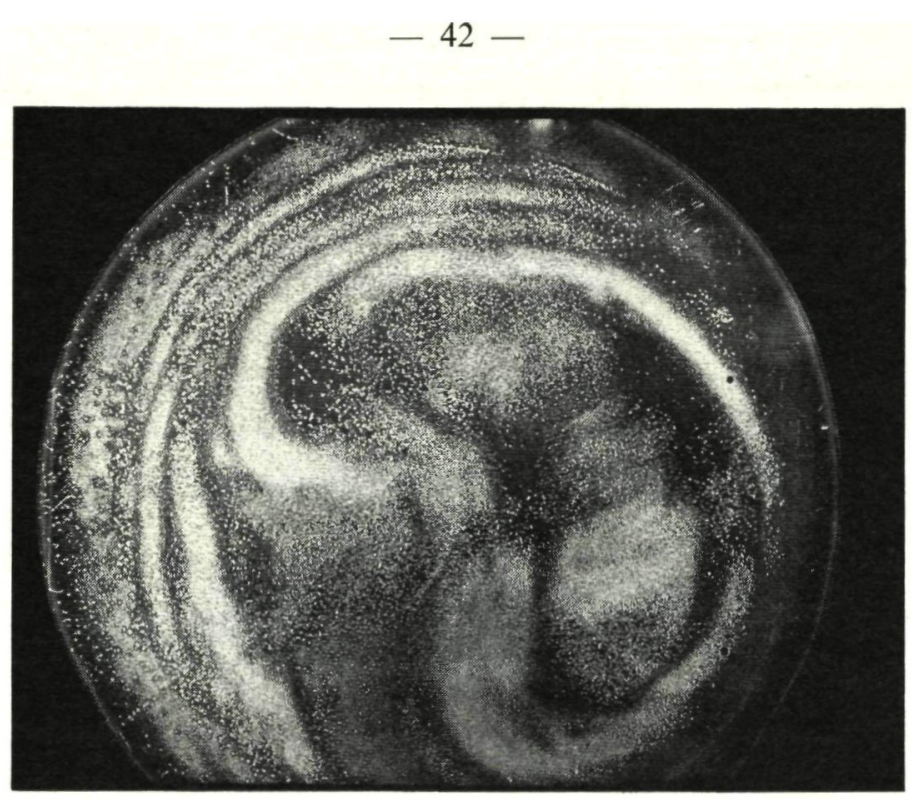

Fig. 3.16. X-ray transmission topograph of a copper-decorated cross-section of a crystal grown in the $\langle 111\rangle$ direction. Crystal diameter: $27 \mathrm{~mm}$; $(2 \overline{2} 0)$ reflex. Non-circular symmetric defect distribution.

\subsection{Comments on the results obtained with etching and copper decoration}

\section{(a) Striated pattern}

In longitudinal sections preferential etching as well as copper decoration reveal that the microdefects are located in a layer pattern, whereas in crosssectional slices the defects are present in a pattern of more or less circular bands. Combining both patterns, it can be concluded that the spatial defect distribution in the crystal has the form of a spiral ramp, exhibiting randomly distributed interruptions. Because of these interruptions the separations between the layers, visible in the longitudinal slices, vary. However, the average minimum separation equals $V_{g} / R^{\prime}$. The concentration of the defects in the region in between the spiral ramp is very low, sometimes even zero (e.g. fig. 3.15).

\section{(b) Solid-liquid interface}

The defect-layer pattern reflects the shape of the solid-liquid interface as is shown by the relation existing between dope striations and defect distribution (sec. 3.2). Consequently, the shape of the solid-liquid interface can be studied by revealing the microdefects. The defect-layer pattern greatly depends on the crystal-growth parameters, such as growth rate, crystal orientation and diameter. An interface, slightly convex towards the melt, is found in the crystals grown in argon at $1-2 \mathrm{~mm} / \mathrm{min}$ with $\langle 111\rangle$ growth direction (fig. 3.7). Con- 
sequently, a central (111) facet will form ${ }^{3-2,3}$ ). On those facets (fig. 3.7(c)) the defect layers are generally less sharply defined than in the regions where the interface is curved. If the growth rate is increased to $3 \mathrm{~mm} / \mathrm{min}$ (fig. $3.1 \mathrm{l}(b)$ ) the interface becomes strongly concave towards the melt. This is mainly due to the increase of heat flow towards the crystal surface caused by the increase in the latent heat production per second at the fast-growing interface, resulting in a stronger curvature of the freezing isotherm.

Convex interfaces in $\langle 100\rangle$-oriented crystals (fig. $3.12(b)$ ) will exhibit no facetting effects.

As can be expected, the microdefect pattern revealed in thin cross-sectional slices is closely related to the interfacial curvature. For a strongly curved interface this pattern consists of a number of interrupted circular bands (figs 3.11(a) and $3.12(a)$ ). In the case of a rather flat interface, however, these bands broaden and become less numerous (fig. 3.10).

In the crystal region IV (fig. 3.7(c)) the crystal diameter is reduced to zero by gradually increasing the melt temperature, which leads to rather complicated growth conditions. Among other things this procedure causes a shift of the freezing isotherm in the upward direction and consequently an upward move of the solid-liquid interface. This introduces large growth-rate variations. Although the seed is still withdrawn in the vertical direction at a speed of $2 \mathrm{~mm} / \mathrm{min}$ in this case, the shift of the interface causes a decrease of the average growth rate. The shape of the defect striations indicates that even remelt $(V<0)$ occurs. The reduced growth rate causes the interface to become extremely convex towards the melt with a large central (111) facet. Simultaneously a steep rise in the cluster concentration is observed in this region.

\section{(c) Cluster type}

It was established by preferential etching that in dislocation-free F.Z. silicon two types of microdefects are present, which differ in size and concentration. In the bulk of the crystals both types are present and are distributed in the same striated pattern (fig. 3.4), while only the small ones are found in a thin surface region and in a part of crystal region II. In the bulk of the crystal copper decoration reveals only the larger defects (figs 3.7, 3.9 and 3.10), while the smaller ones are only decorated in the surface and cone regions where the larger ones are absent (figs 3.8 and $3.13(a)$ ).

The upper part of crystal region II (fig. 3.12(b)) as well as region I, are usually completely free of defects. In crystals grown in vacuum instead of in argon no small defects are detected by copper decoration (compare figs 3.14 and 3.15 with figs $3.7,3.8,3.10,3.11,3.12$ ).

Henceforth we will call the larger defects A-clusters and the smaller ones B-clusters. 


\section{(d) Concentration}

The concentration of the decorated defects could readily be determined by counting the direct images of these imperfections visible on the topographs made of the cross-sectional slices, taking into account the thickness of these samples. Every single defect is detected in this way. Consequently, microdefect concentrations in the range of $0-10^{7} \mathrm{~cm}^{-3}$ can be measured. At the upper limit $\left(10^{7} \mathrm{~cm}^{-3}\right)$ the strain fields of the decorated defects begin to overlap, making an exact counting difficult.

The average concentration of the A-clusters measured in the majority of the crystals grown either in argon or vacuum varied between $5.10^{5}$ and $2.10^{6}$ $\mathrm{cm}^{-3}$; the highest concentration was found in a commercially available floatingzone crystal and amounted to about $10^{7} \mathrm{~cm}^{-3}$. The exact growth conditions of this crystal were unknown.

The local concentration of decorated B-clusters in the surface region of the material grown in argon was about $2.10^{6} \mathrm{~cm}^{-3}$. From the etching results, shown in fig. 3.4, it was concluded that in the bulk we have: $N_{\mathrm{B}} \approx 15 N_{\mathrm{A}}$.

Neither the level of doping $\left(0-6 \cdot 10^{16} \mathrm{~cm}^{-3}\right)$ nor the orientation of the crystals ((111) or (100)) had any detectable influence on the average defect concentration. The radial variation in the concentration of decorated defects is schematically plotted in fig. 3.17. Both preferential etching and copper decoration showed that the A-cluster concentration often exhibited a minimum in the central area of the crystal. This minimum is very pronounced in the crystals shown in figs 3.11 and 3.15, yet it is almost absent in some other crystals.

Comparison of the infrared micrographs shown in figs $3.8(b), 3.9(b)$ and 3.13(b) with the corresponding X-ray topographic details (fig. 3.8(a), 3.9(a) and $3.13(a)$ ) shows that both methods detect the same defect configuration.

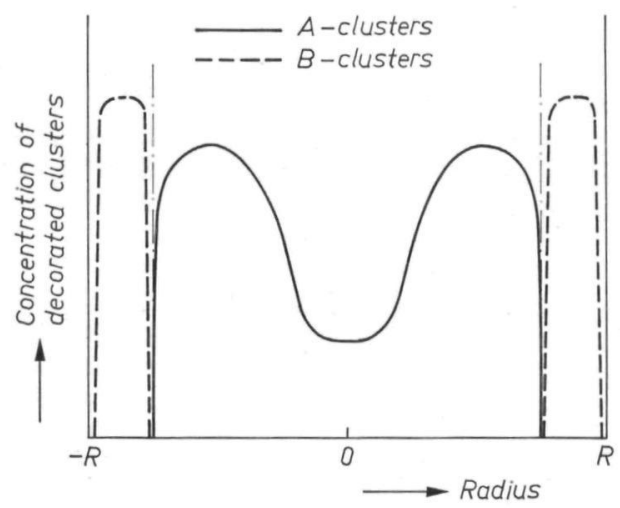

Fig. 3.17. Concentration profile of the copper-decorated A- and B-clusters as observed in a longitudinal section of an argon-grown crystal. 


\section{(e) Precipitate structure}

From the infrared transmission micrographs (figs 3.8(b), 3.9(b) and 3.13(b)) it can be concluded that the copper precipitates formed on the A- and Bclusters show the well-known ${ }^{3-4,5,6}$ ) dendritic fine structure.

\section{(f) Facet formation}

In contrast with $\langle 100\rangle$-oriented crystals, dislocation-free crystals grown in the $\langle 111\rangle$-direction usually have a non-circular cross-section (fig. 3.11(a)). One or more ridges, which are about parallel to the growth axis, are formed on the outside of the crystals. In agreement with a study reported by Ciszek ${ }^{3-7}$ ), it was established that at the position of these ridges (111) interfacial facets are formed. As soon as dislocations are introduced during growth the interfacial facets together with the asymmetric shape of the crystal disappear abruptly. This gives the opportunity to determine by visual inspection whether the growing crystal remains dislocation-free.

\section{(g) Influence of dislocations}

Figure 3.18 shows a microscopic detail of the edge region at $\mathrm{E}$ in fig. 3.10. During the copper-diffusion procedure an array of dislocations was introduced

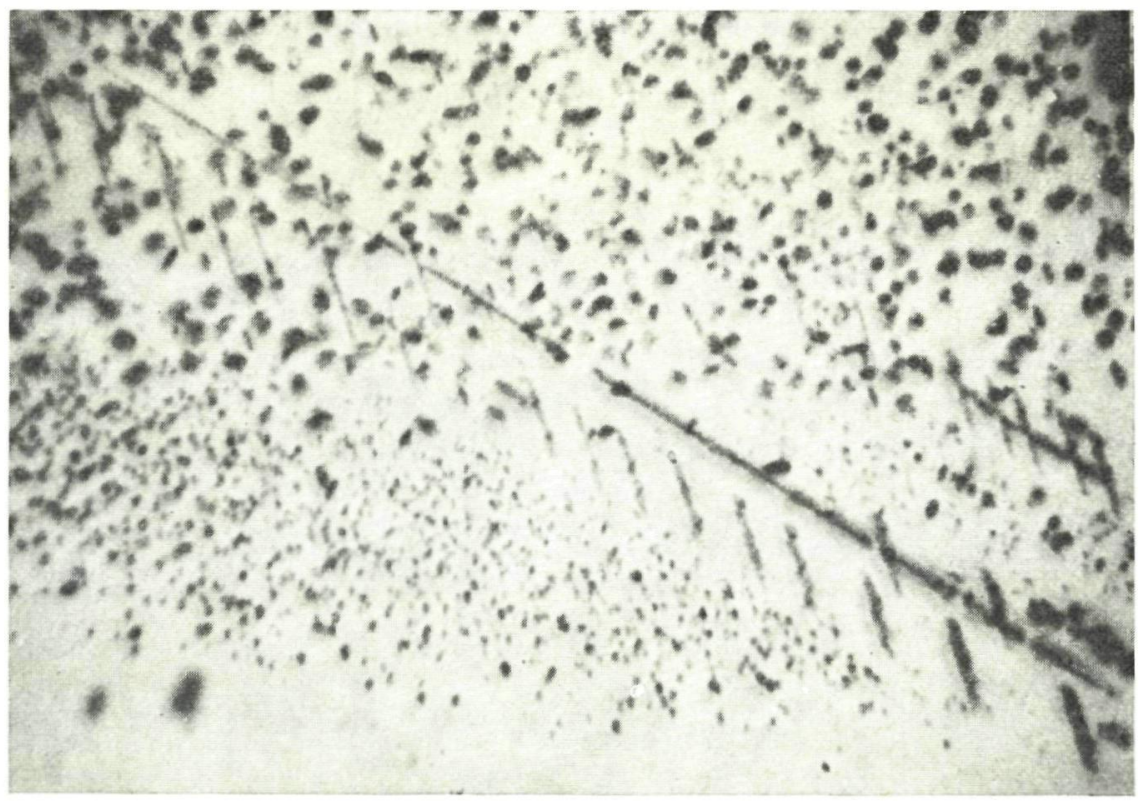

Fig. 3.18. Microscopic detail of the topograph in fig. 3.10 at E. Dislocations, introduced during decoration, influence the copper precipitation on the microdefects. 
by plastic deformation*). This array extends from the edge over several $\mathrm{mm}$ into the slice. It can be noticed that the dislocations present in the edge region are heavily decorated. Those B-clusters present in this region in the direct neighbourhood of the array are not decorated. On the other hand a weak decoration of the dislocations is observed in the area where A-clusters are found, whereas the precipitation of copper on these A-clusters is not influenced by the presence of the line defects. These observations indicate that the interaction between copper impurities and dislocations is stronger than the interaction of these impurities with the B-clusters, but weaker than the interaction between copper impurities and the A-clusters.

\subsection{Results obtained with lithium decoration}

The decoration procedure has been described in sec. 2.4. The lithium diffusion was carried out at temperatures between 400 and $600{ }^{\circ} \mathrm{C}$. After diffusion the samples were either quenched or slowly cooled to room temperature. Finally, an annealing treatment at $160^{\circ} \mathrm{C}$ enabled the supersaturated amount of lithium to precipitate on the nucleation sites present. This annealing temperature was chosen mainly for the two following reasons. Firstly, with the value of the diffusion coefficient of lithium in silicon at this temperature of $4 \cdot 8 \cdot 10^{-11}$ $\mathrm{cm}^{2} / \mathrm{s}^{3-9}$ ), it could be expected that the precipitation rate would be fast enough to complete the precipitation process in an acceptable period of time. Secondly, the precipitation kinetics may be influenced by ion-pairing phenomena, resulting in the formation of complexes of lithium donors with acceptor impurities present in the material. However, it has been reported by Pell ${ }^{3-10}$ ) that ion-pairing processes in lithium-doped silicon are negligible at temperatures above $150^{\circ} \mathrm{C}$. The precipitation rate was determined by measuring the variation in electrical resistivity with the four-point-probe technique. The amount of lithium, which was still in solution, was derived from the resistivity values in combination with the data given by Irvin ${ }^{3-11}$ ), assuming that the formation of precipitates did not affect the electron mobility. In order to carry out the electrical and $\mathrm{X}$-ray topographic measurements, the annealing treatments were interrupted periodically.

A cross-sectional slice taken from the same pedestal-pulled crystal as the

*) Because the dislocation image contrast depends on the angle between the Burgers vector b of the dislocation and the diffraction vector $\mathbf{g}$, the character of a "clean" dislocation can be determined by making several $\mathrm{X}$-ray transmission topographs using different reflecting planes. Maximum contrast is obtained if $b$ and $g$ are parallel, whereas no image is formed if the two vectors are perpendicular to each other. However, from the broad dislocation images in fig. 3.18 as well as by infrared-microscopic investigation it was established that copper precipitation on the dislocations had taken place. Such a precipitation of a second phase changes the strain field around the dislocation in such a way that the contrast criterion does not hold any more. A contaminated dislocation remains visible irrespective of the direction of $\mathrm{g}$. Consequently, the dislocation type in this array could not be determined. However, it has been reported ${ }^{3-8}$ ) that such dislocations are generally of the $60^{\circ}$-type. 
slice shown in fig. 3.11(a) was subjected to the following treatment. Lithium was diffused at $400{ }^{\circ} \mathrm{C}$ during 5 hours, followed by cooling to room temperature in about 15 minutes. The lithium-donor concentration amounted to $3.10^{17} \mathrm{~cm}^{-3}$. Some lithium would have been precipitated during the relatively long period of cooling. However, X-ray topography showed no contrast, indicating that the precipitates were still very small. Subsequently, the slice was annealed at $160^{\circ} \mathrm{C}$. Simultaneously with an increase in resistivity, images of Li precipitates are revealed by topography. The image contrast continuously increases with increasing annealing time. It is established that lithium precipitates are formed on the microdefects present, resulting in a contrast pattern similar to that observed after copper decoration. Figure 3.19 shows the topo-

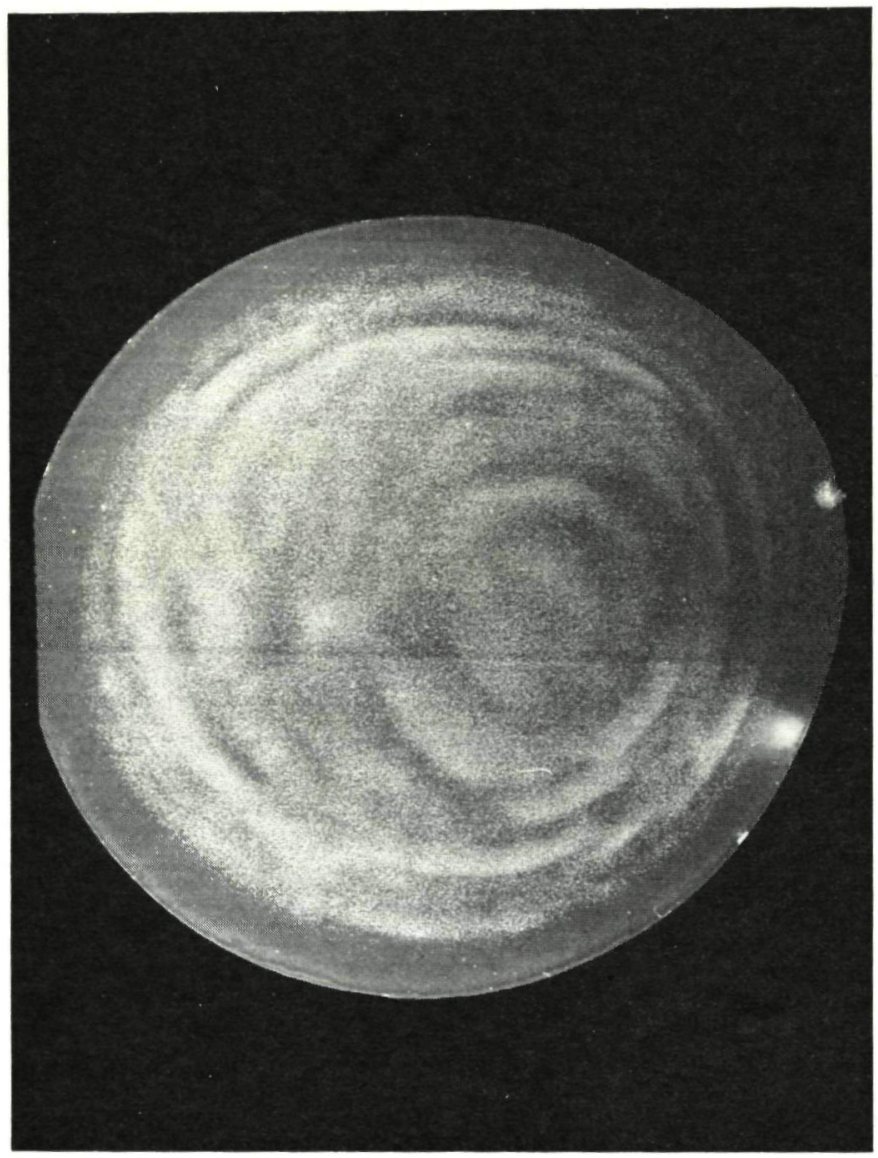

Fig. 3.19. X-ray transmission topograph showing lithium precipitation on the A-clusters. The same argon-grown crystal is used as in fig. 3.11. $T_{D}=400^{\circ} \mathrm{C}$. Slow cooling after diffusion. Annealing at $160^{\circ} \mathrm{C}$ during $238 \mathrm{~h}$; $(2 \overline{2} 0)$ reflex. 
graph made after an annealing period of $238 \mathrm{~h}$, where the lithium-donor concentration has decreased to $10^{16} \mathrm{~cm}^{-3}$. If we compare this result with fig. 3.11(a) it can be concluded that the A-cluster distribution is revealed by lithium decoration, but that the edge region, containing B-clusters only, does not show any images of defects. The concentration of the A-clusters, determined from this topograph, was equal to the one found by copper decoration $\left(1-2.10^{6} \mathrm{~cm}^{-3}\right)$. A similar result was obtained when a sample of this crystal was quenched after diffusion at $400{ }^{\circ} \mathrm{C}$ and subsequently annealed at $160{ }^{\circ} \mathrm{C}$. It appeared, however, that the precipitation process was strongly affected by the diffusion temperature $\left(T_{D}\right)$. For, in contrast to diffusions at $T_{D} \leqslant 400{ }^{\circ} \mathrm{C}$, it was found that with $T_{D}>400{ }^{\circ} \mathrm{C}$ lithium not only precipitated on the grown-in vacancy clusters but also on a high concentration of new nucleation sites which were generated by the decoration procedure itself. At $T_{D} \approx 500{ }^{\circ} \mathrm{C}$ nucleation sites are formed during the lithium diffusion, whereas at $T_{D} \geqslant 550{ }^{\circ} \mathrm{C}$ nuclei are formed after quenching to room temperature. The lithium precipitation on these new nucleation sites dominated the precipitation process. Some aspects of these processes of nucleus generation, which are still being studied in greater detail, will be discussed in chapter 5. Because of this the electrical measurements were carried out in combination with $T_{D} \leqslant 400^{\circ} \mathrm{C}$. Figure 3.20 shows

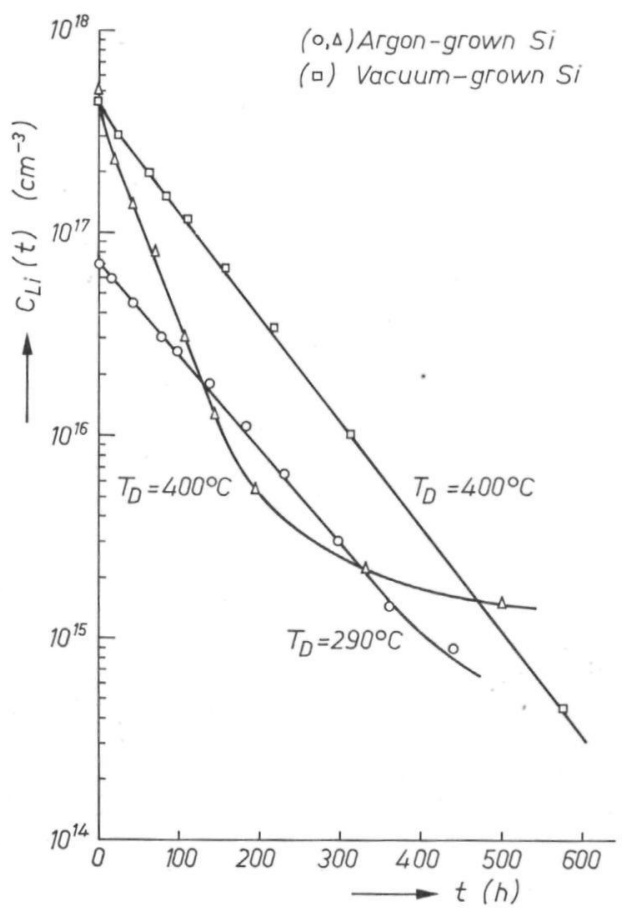

Fig. 3.20. The decrease of the electrically active lithium content as a function of the annealing time after diffusions at 290 and $400{ }^{\circ} \mathrm{C}$ in argon-grown and vacuum-grown dislocation-free silicon crystals. Annealing temperature: $160^{\circ} \mathrm{C}$. 
the decrease in unprecipitated lithium content as a function of annealing time after diffusion into slices of crystals grown in argon and vacuum, respectively. The argon-grown crystal is identical with the one shown in figs 3.2, 3.11 and 3.19 while the vacuum-grown crystal is identical with the one shown in fig. 3.14. Diffusion temperatures of 290 and $400{ }^{\circ} \mathrm{C}$ were used while the samples were quenched to room temperature after diffusion. The precipitation curves exhibit simple exponential decay almost from the beginning of the precipitation process.

When a diffusion temperature of about $600{ }^{\circ} \mathrm{C}$ was combined with slow cooling of the samples to room temperature instead of quenching, a strong lithium decoration of the vacancy clusters was obtained, which occurred already during the cooling procedure. Precipitation on new sites was not detected in this case. This decoration procedure is therefore similar to the one used for copper decoration. When samples, treated in this way, were annealed at $160^{\circ} \mathrm{C}$ afterwards, the decoration of the clusters became still stronger, while precipitation on new sites often remained of minor importance.

Figure 3.21 shows the topograph of half a cross-section of the same argongrown crystal as shown in figs 3.11 and 3.19 . after diffusion at $600{ }^{\circ} \mathrm{C}$ during $5.5 \mathrm{~h}$ followed by slow cooling in about $15 \mathrm{~min}$ to room temperature. The measured unprecipitated amount of lithium was $5 \cdot 4 \cdot 10^{18} \mathrm{~cm}^{-3}$. A heavy decoration of the A-clusters, which has taken place during the cooling period, is revealed, whereas again the B-clusters remain undetected.

The lithium precipitation has also been investigated by infrared microscopy. It was established that the precipitates formed on the grown-in A-clusters have the shape of flat circular disks (see fig. 3.22). Consequently, the infrared images of those disks, which are not parallel to the sample surface, are elliptic in shape with the ratio of the two axes determined by the orientation of the disks.

Some lithium-precipitation experiments were carried out on dislocated pedestal-pulled crystals. In agreement with results reported by Takabatake et al. ${ }^{3-12}$ ), decoration of the dislocations was observed (see fig. 3.23). From the images of those segments of the dislocations which were in focus under the infrared microscope, it could be concluded that discrete lithium precipitates were formed along the dislocation lines. No grown-in microdefects were found.

\subsection{The influence of microdefects on the performance of micro-electronic devices}

\subsubsection{Introduction}

Planar micro-electronic devices, such as diodes and transistors, are made by successive oxidation and diffusion treatments in the surface layer of slices taken from silicon single crystals. These process treatments are usually carried out at temperatures between 900 and $1200{ }^{\circ} \mathrm{C}$ in inert and oxidizing atmospheres. Most types of discrete planar devices or integrated circuits are made in layers, 


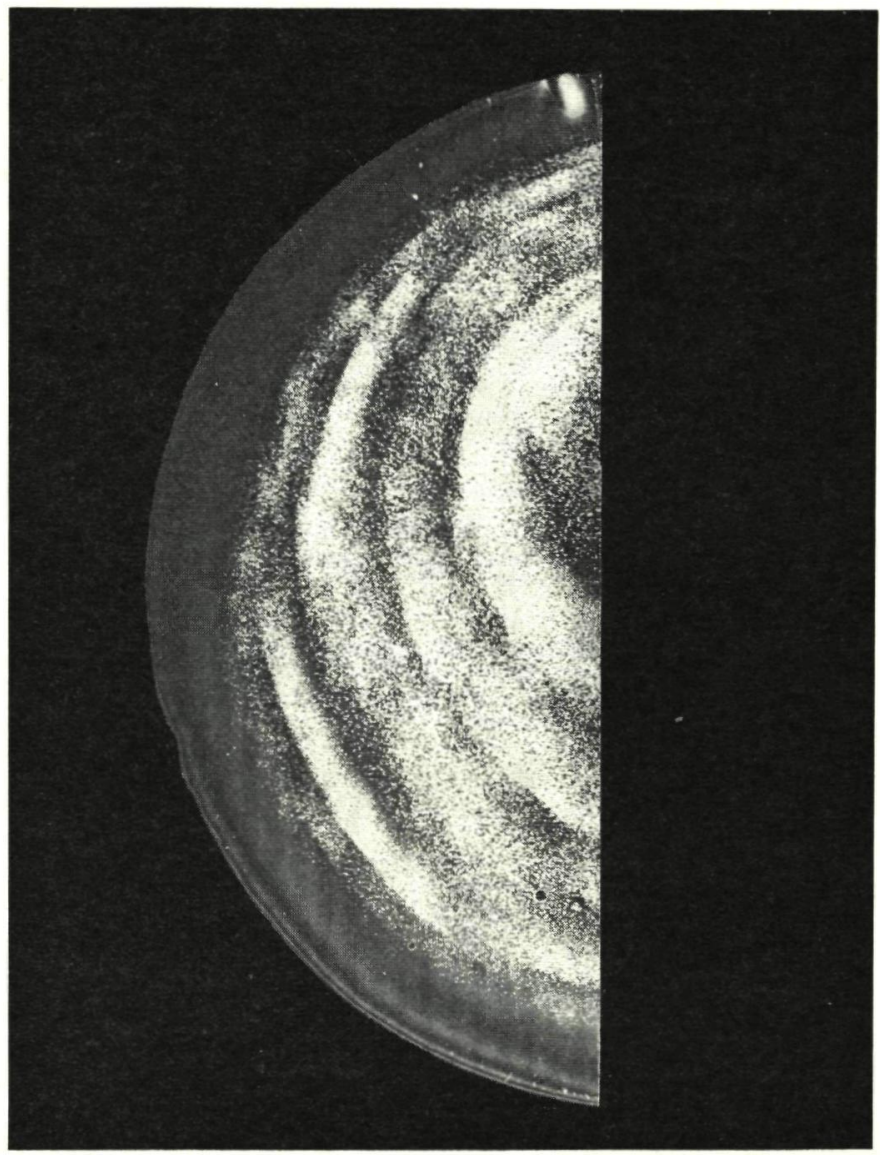

Fig. 3.21. X-ray transmission topograph of half a cross-section taken from the same argongrown crystal as shown in figs 3.11 and 3.19 , after lithium diffusion. $T_{D}=600{ }^{\circ} \mathrm{C}$; $(2 \overline{2} 0)$ reflex. Lithium precipitates on the A-clusters during cooling in $15 \mathrm{~min}$ to room temperature. 


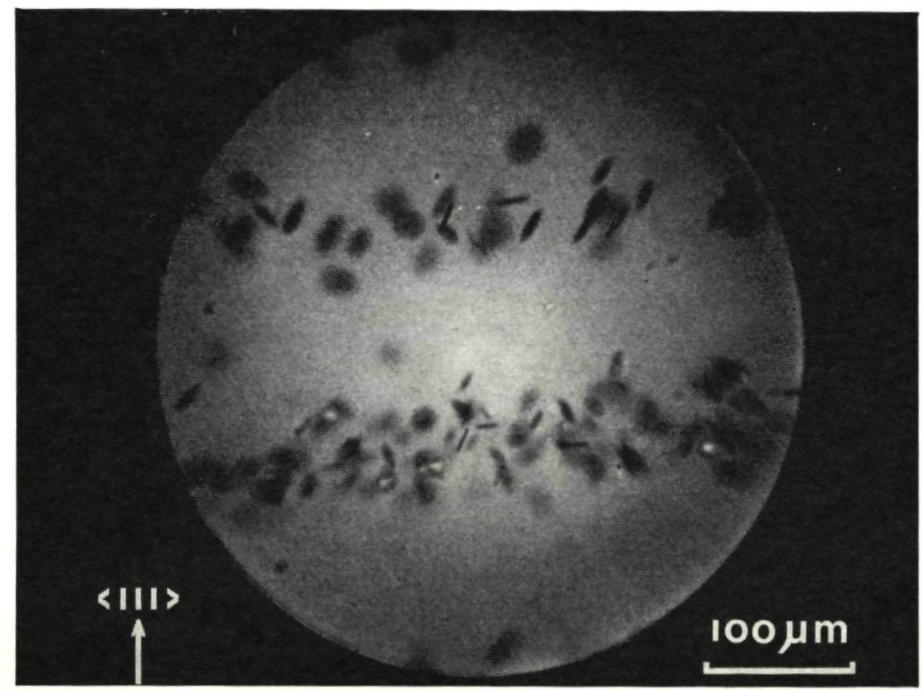

Fig. 3.22. Infrared transmission micrograph showing disk-shaped precipitates on the Aclusters. Vacuum-grown material. $T_{D}=600^{\circ} \mathrm{C}$. Slow cooling after diffusion. Annealing time at $160{ }^{\circ} \mathrm{C}: 120 \mathrm{~h}$.

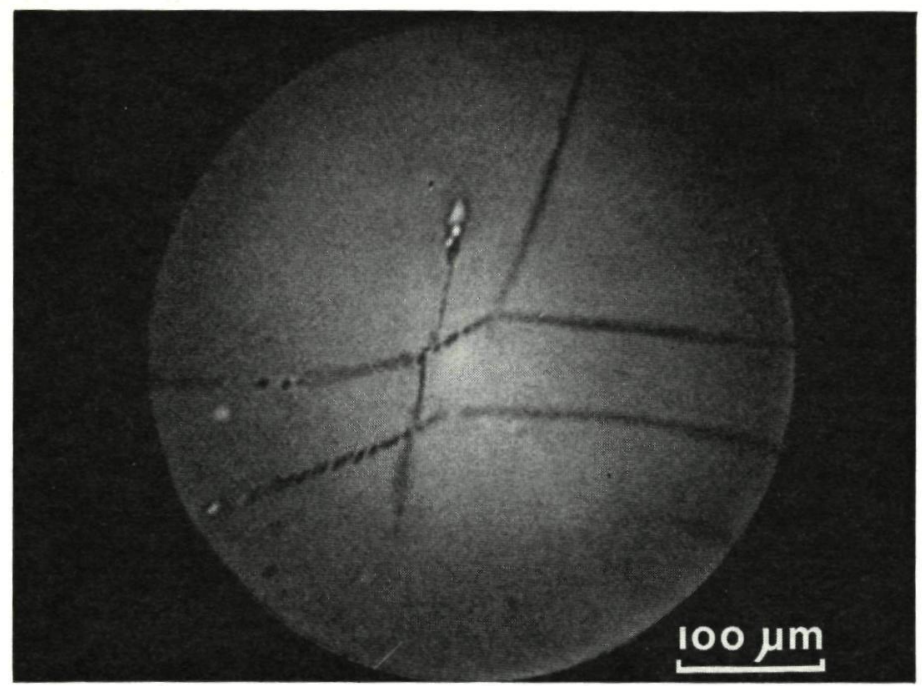

Fig. 3.23. Infrared micrograph showing lithium precipitation on dislocations in the form of discrete particles. $T_{D}=600^{\circ} \mathrm{C}$. Slow cooling after diffusion. Annealing time at $160{ }^{\circ} \mathrm{C}$ : $101 \mathrm{~h}$. 
epitaxially grown from the vapour phase on the above-mentioned slices. This epitaxial-growth process requires a sample temperature between about 1000 and $1250^{\circ} \mathrm{C}$. Some devices, however, such as, for instance, the diode arrays used in silicon-vidicon television pick-up tubes ${ }^{3-13}$ ) and the so-called MOS transistors (MOS stands for Metal-Oxide-Semiconductor) are generally made on the melt-grown material itself.

When dislocation-free high-purity silicon crystals are used, it can be expected that the various high-temperature treatments applied in planar-device technology will have a certain impact on the character and distribution of the microdefects present. This has been investigated in a limited number of experiments. Furthermore, the influence of the microdefects on the perfection of epitaxial layers as well as the influence on the electrical performance of planar diodes have been studied.

\subsubsection{Annealing experiments in nitrogen}

Slices taken from dislocation-free crystals containing A- and B-clusters were subjected to annealing treatments at temperatures between 1000 and $1200{ }^{\circ} \mathrm{C}$ in nitrogen. These high-temperature treatments were carried out in tubular furnaces normally used for device processing. After annealing, the samples were investigated by preferential etching and $\mathrm{X}$-ray topography.

It was observed that after annealing some of the clusters had changed into stacking faults which were located in the various (111) planes. This could be concluded from the shape of the etch pits formed on the surface of (111) cross-sectional slices. It is known ${ }^{3-14,15}$ ) that stacking faults located in (111) planes other than the one which has been etched, give rise to the formation of trapezoid etch pits. Two dislocation etch pits are often visible at the ends of the trapezoid indicating the presence of the surrounding dislocation loop. Most of the clusters, however, did not change into such planar defects. Irregular etch pits and hillocks were formed at these clusters which seems to indicate that impurity precipitation on these defects had occurred. Resistance-heated furnaces always contain relatively large amounts of metallic impurities. It is likely, therefore, that precipitation of some of these metallic impurities on the defects had taken place, preventing the clusters from changing into stacking faults. In a few cases the contamination was so severe that no stacking faults were formed at all.

In order to study the influence of heat treatments under more-pure conditions, some samples were coated with a layer of phosphorus glass by deposition and diffusion of phosphorus at $1050{ }^{\circ} \mathrm{C}$ in an oxidizing atmosphere. Such a layer prevents the penetration of metallic impurities into the samples during subsequent heat treatments ${ }^{3-16.17}$ ). It was found that under these conditions a large part of the A-clusters in the bulk of the samples changed into stacking faults (see figs 3.24 and 3.25). Figure 3.24 shows the etch pits formed 


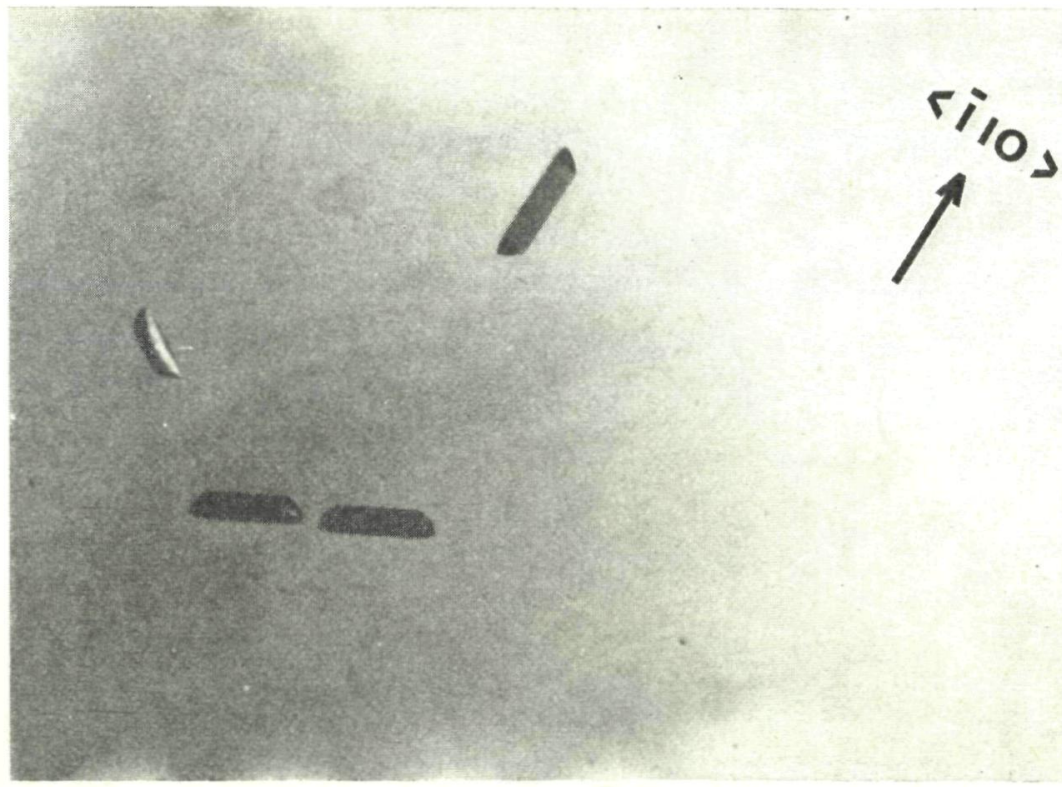

Fig. 3.24. Dislocation-free cross-sectional slice annealed for $4 \mathrm{~h}$ at $1150^{\circ} \mathrm{C}$ in nitrogen. Slice coated with phosphorus glass. After annealing a surface layer of about $100 \mu \mathrm{m}$ is removed by etching, followed by preferential etching. Trapezoid etch pits caused by stacking faults are observed. Magnification: $500 \times$.

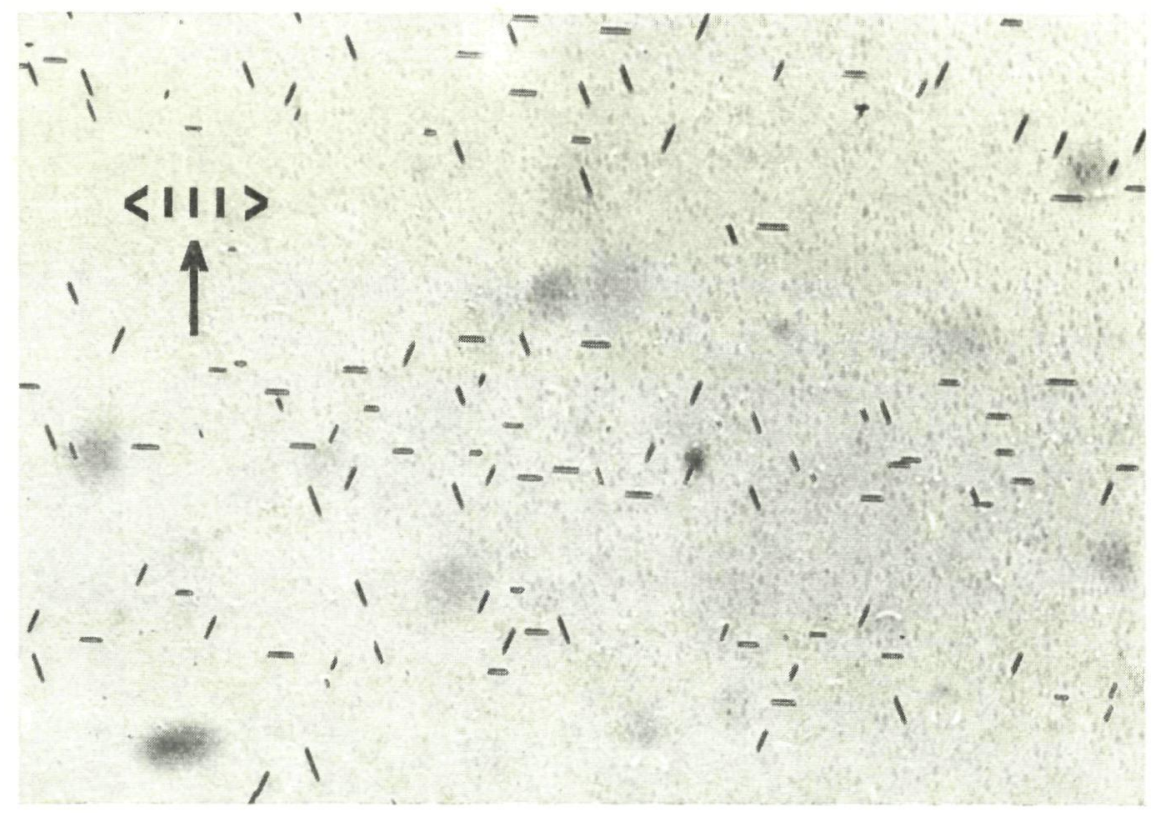

Fig. 3.25. Longitudinal section of a dislocation-free crystal. Similar treatment as in fig. 3.24. Magnification: $200 \times$. 
on the (111) cross-sectional surface taken from a crystal grown in the $\langle 111\rangle$ direction. Prior to preferential etching, the sample had been non-preferentially etched in order to remove a surface layer of about $100 \mu \mathrm{m}$. The trapezoid pits are clearly visible. As can be expected these trapezoids are oriented along the (110), $(0 \overline{1} 1)$ and (10) directions, thus making angles of $120^{\circ}$ with each other. Of the planar faults present in the (111) cross-sectional surface itself, only the dislocation loops are revealed. These loops are found to be circular in shape.

In X-ray transmission topographs (fig. 3.26) the stacking faults cause strong direct-image contrast without any decoration. Apart from the large stacking faults originating from the A-clusters, a striated distribution of much smaller stacking faults was revealed by preferential etching as well as X-ray topography. These small ones are also present in the surface region where only B-clusters are present. It is therefore concluded that the B-clusters change into stacking faults as well. The stacking faults cause the total number of defect images (fig. 3.26) to be much higher than that visible in the topographs after copper decoration.

The diameter of the stacking faults increased with increasing annealing time and temperature. It was not established whether the stacking faults were intrinsic or extrinsic in character (see fig. 3.27).

\subsubsection{Oxidation}

Silicon slices containing A- and B-clusters were oxidized at elevated temperatures in a flow consisting of a mixture of nitrogen and water vapour (wet oxidation) or nitrogen and oxygen (dry oxidation). It was established that after wet oxidation those clusters located near the $\mathrm{Si}-\mathrm{SiO}_{2}$ interface changed into stacking faults (figs 3.28 and 3.29). The size of these planar defects increased with the oxidation time and became for instance up to $200 \mu \mathrm{m}$ in diameter after $16 \mathrm{~h}$ of wet oxidation at $1150{ }^{\circ} \mathrm{C}$ (fig. 3.28). In the bulk of the samples stacking faults were found, which were much smaller than those formed at the $\mathrm{Si}-\mathrm{SiO}_{2}$ interface. In some experiments stacking-fault formation was much less pronounced. In these cases the irregular shape of the etch pits again seemed to indicate that contamination with impurities had taken place.

Similar stacking faults were formed during dry oxidation. The size and concentration of the stacking faults, however, were smaller than after a comparable wet-oxidation treatment.

\subsubsection{Epitaxy}

Epitaxial silicon layers of about $9 \mu \mathrm{m}$ thickness were grown on one side of dislocation-free substrates containing $\mathrm{A}$ - and B-clusters. The growth process was carried out in an r.f.-heated epitaxial reactor by reduction of $\mathrm{SiCl}_{4}$ at $1225^{\circ} \mathrm{C}$ in a hydrogen flow. The crystal perfection of both the layers and the substrates was examined. Preferential etching and X-ray topography revealed 

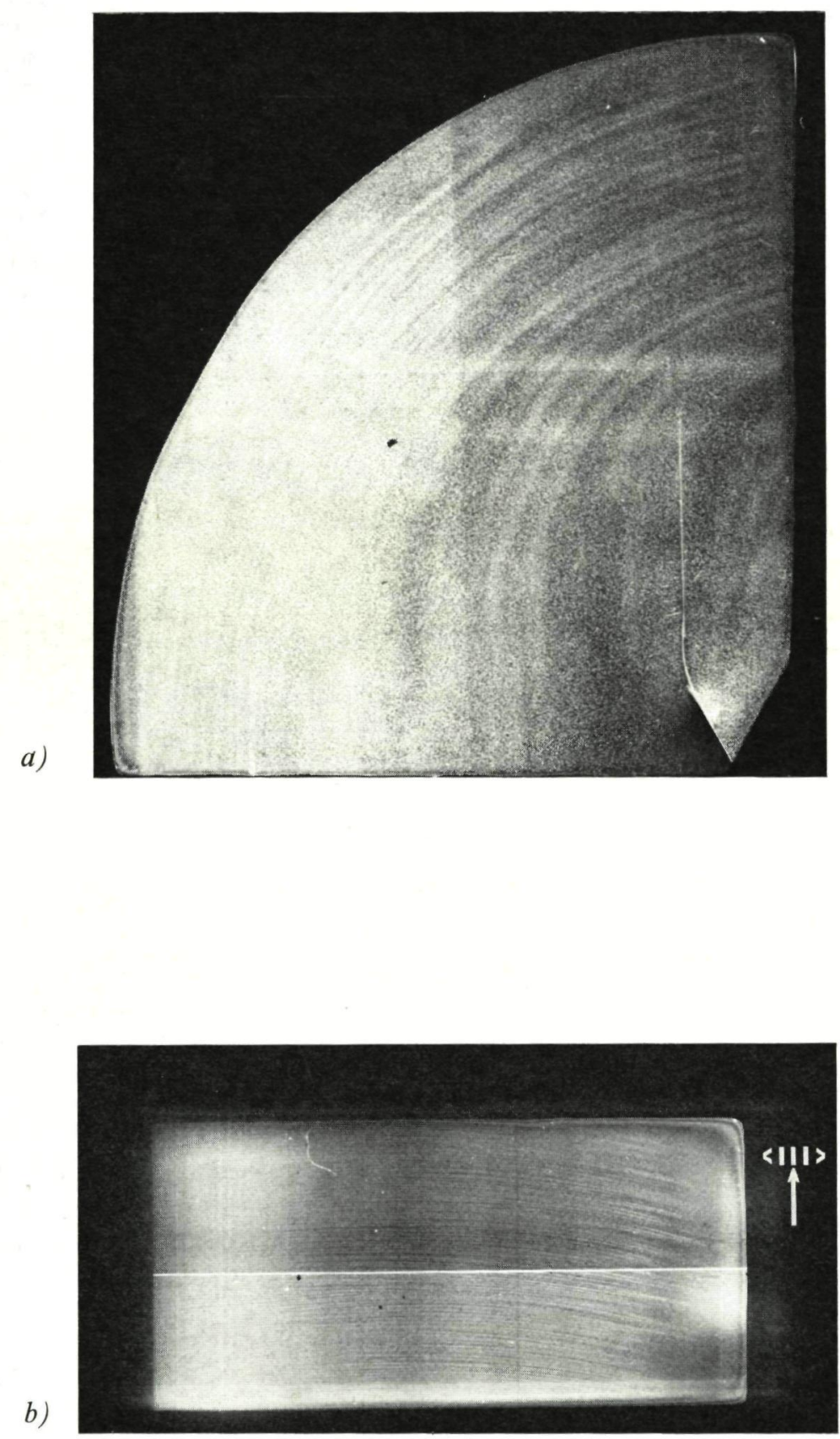

Fig. 3.26. X-ray transmission topographs of samples which are annealed during $4 \mathrm{~h}$ at $1150{ }^{\circ} \mathrm{C}$ in nitrogen. Samples covered with phosphorus glass prior to annealing. (a) Part of a cross-section; average concentration of defects: $>10^{7} \mathrm{~cm}^{-3}$. (b) Part of a longitudinal section; images of both A- and B-clusters are visible without decoration. 
a)
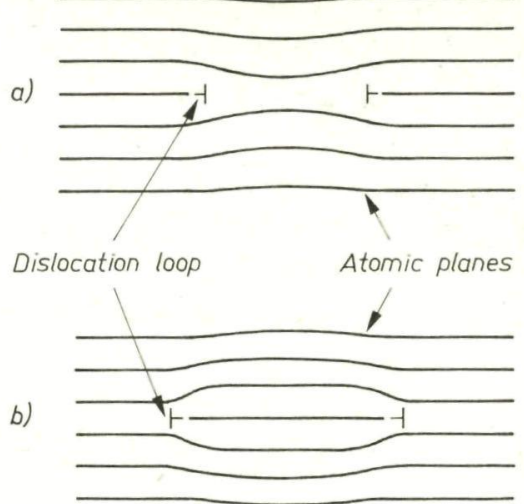

Fig. 3.27. Stacking faults of intrinsic $(a)$ and extrinsic $(b)$ nature.

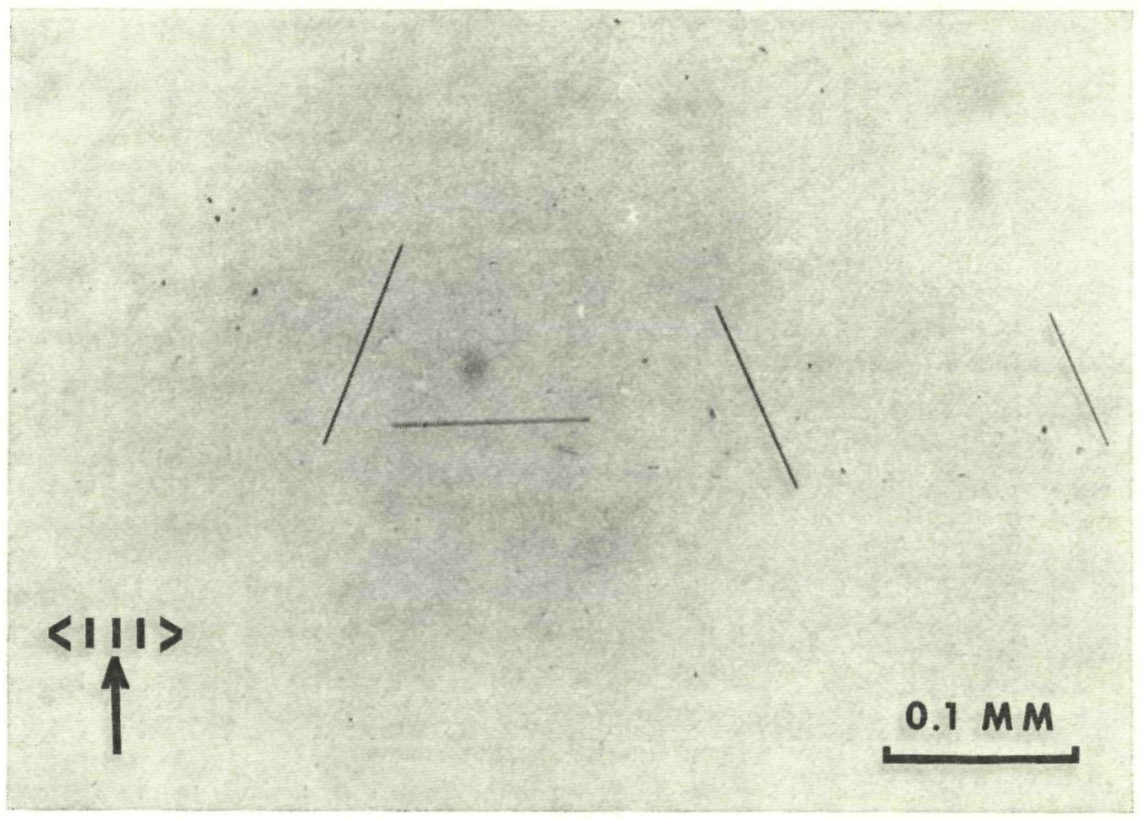

Fig. 3.28. Large stacking faults formed at the $\mathrm{Si}-\mathrm{SiO}_{2}$ interface after wet oxidation at $1150{ }^{\circ} \mathrm{C}$ for $16 \mathrm{~h}$ as revealed by preferential etching. Magnification: $200 \times$. Longitudinal section. 


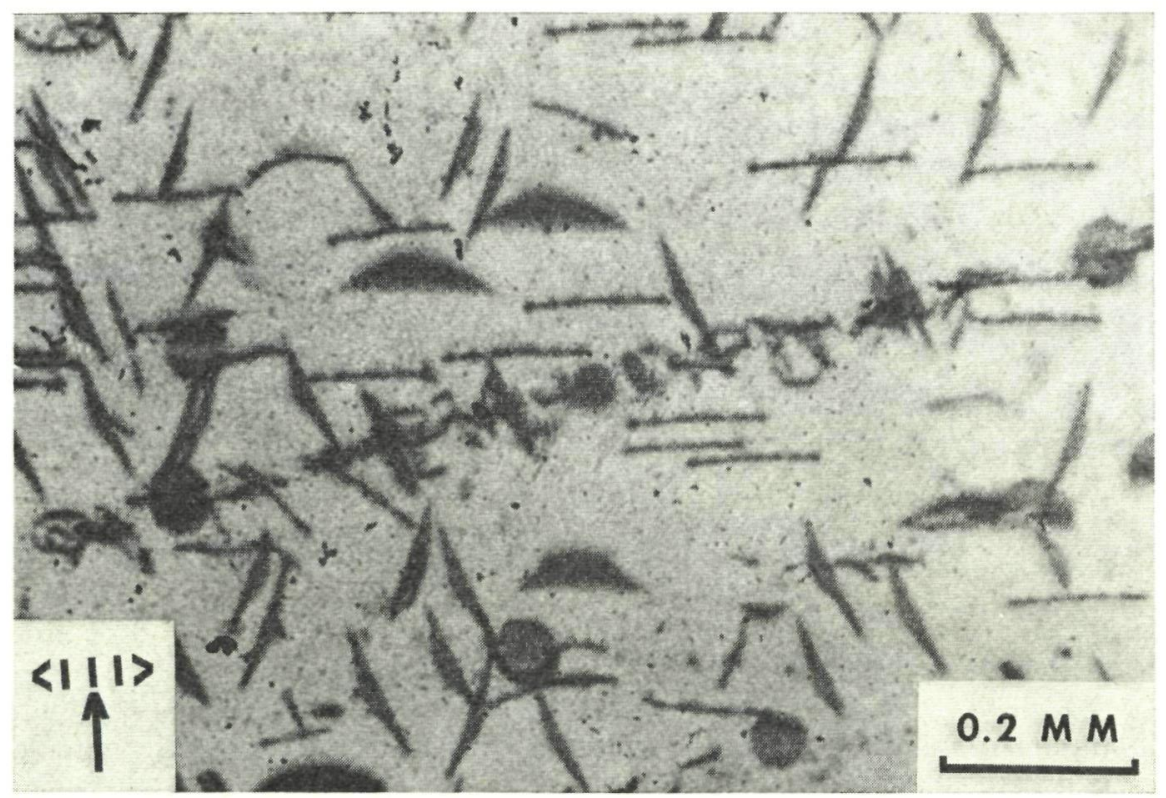

Fig. 3.29. X-ray transmission topographic detail of the same sample of fig. 3.28 ; (111) reflex.

no defects in the epitaxial layers. This seems to indicate that the microdefects present in the substrate did not influence the perfection of the layers. Preferential etching of the back of the substrates or of the front after removal of the epitaxial layer did not reveal the cluster distribution anymore. Moreover, no defect contrast could be seen in topographs made of the substrate material. This not only indicates the absence of stacking-fault formation but also that the strain field surrounding the original microdefects is changed in such a way that no enhanced etching takes place at these defects. However, copper decoration in combination with X-ray topography still revealed a defect distribution similar to that observed before epitaxy. The concentration of the decorated defects, however, was sometimes lower than that found in the as-grown material.

\subsubsection{The influence of microdefects on the properties of planar diodes}

The determination of a possible relationship between the electrical performance of planar diodes and the presence of crystal imperfections requires statistical measurements on a large number of such diodes. This would be very time-consuming if the diodes were investigated one by one. By making use of a special device, the so-called silicon-vidicon ${ }^{3-13}$ ), it was possible to study the leakage current of 360000 planar diodes at a time.

The principle of this device is schematically shown in fig. 3.30. On a thin 


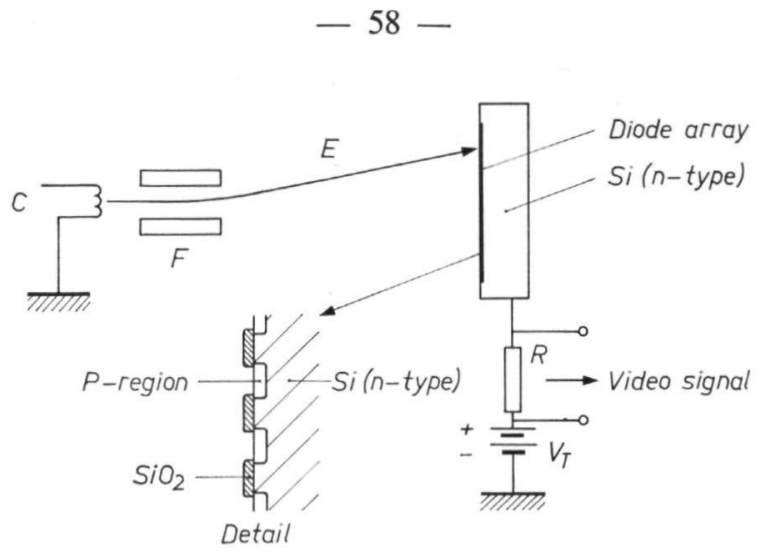

Fig. 3.30. Scheme of the silicon-vidicon pick-up tube. C: cathode of the electron gun; F : focussing and deflection system; E: electron beam.

$n$-type silicon slice an array of 360000 diodes is made by standard photolithographic and planar processing techniques. The centre-to-centre spacing of the circular diodes amounted to $20 \mu \mathrm{m}$. The silicon slice is mounted as target in a vidicon-type ${ }^{3-18}$ ) pick-up tube in which it is scanned by a lowvelocity electron beam. The slice itself is biased positively with respect to the cathode of the electron gun. The voltage between cathode and target $\left(V_{\mathrm{T}}\right)$ is of the order of 5 to 15 volts. The impinging electron beam deposits electrons on the $p$-type diode regions. Since the resistivity of the $\mathrm{SiO}_{2}$ layer is very high, the electronic charge accumulates on this surface and brings this surface up to a potential which is very close to the cathode potential, where it remains if the leakage current of the reverse-biased diodes is very low. Leaky diodes, however, lose their electric charge, causing a leakage current through the resistance $R$. The voltage drop over this resistance, which is associated with the leakage current, is used as the video signal. In this way an overall picture of the distribution of leaky diodes present in the array is obtained on a monitor. The regions containing leaky diodes appear as bright spots. Two examples of such pictures are shown in figs 3.31 and 3.32. Circular interrupted patterns of bright spots are revealed. In addition, a background pattern in the form of a spiral often becomes visible at such target voltages $\left(V_{\mathrm{T}}\right)$ where the formation of a depletion layer at the $\mathrm{SiO}_{2}-\mathrm{Si}$ interface begins (fig. 3.32). This background spiral pattern is probably due to an interaction between metallic impurities and microdefects generated during device processing ${ }^{3-19}$ ). After investigation of the leakage-current pattern, the silicon slice was removed from the tube and its crystal perfection was examined by preferential etching and copper decoration. It was established that the distribution of A-clusters present in the diode surface region was identical to the distribution of the leaky diodes (bright spots). In some cases a fraction of these clusters had changed into stacking faults 


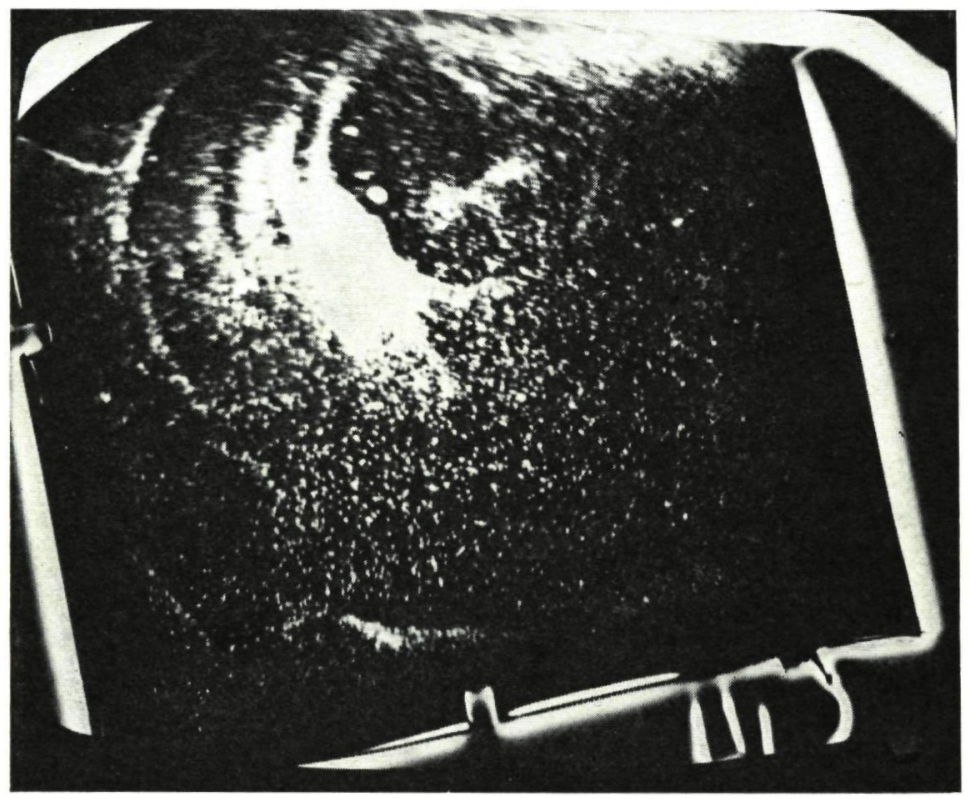

Fig. 3.31. Monitor picture obtained with a target of dislocation-free silicon. Material resistivity: $0 \cdot 25 \Omega \mathrm{cm}$. $n$-type. Target not illuminated. $V_{\mathrm{T}}=13 \mathrm{~V}$. Leaky diode regions are visible as bright spots. 


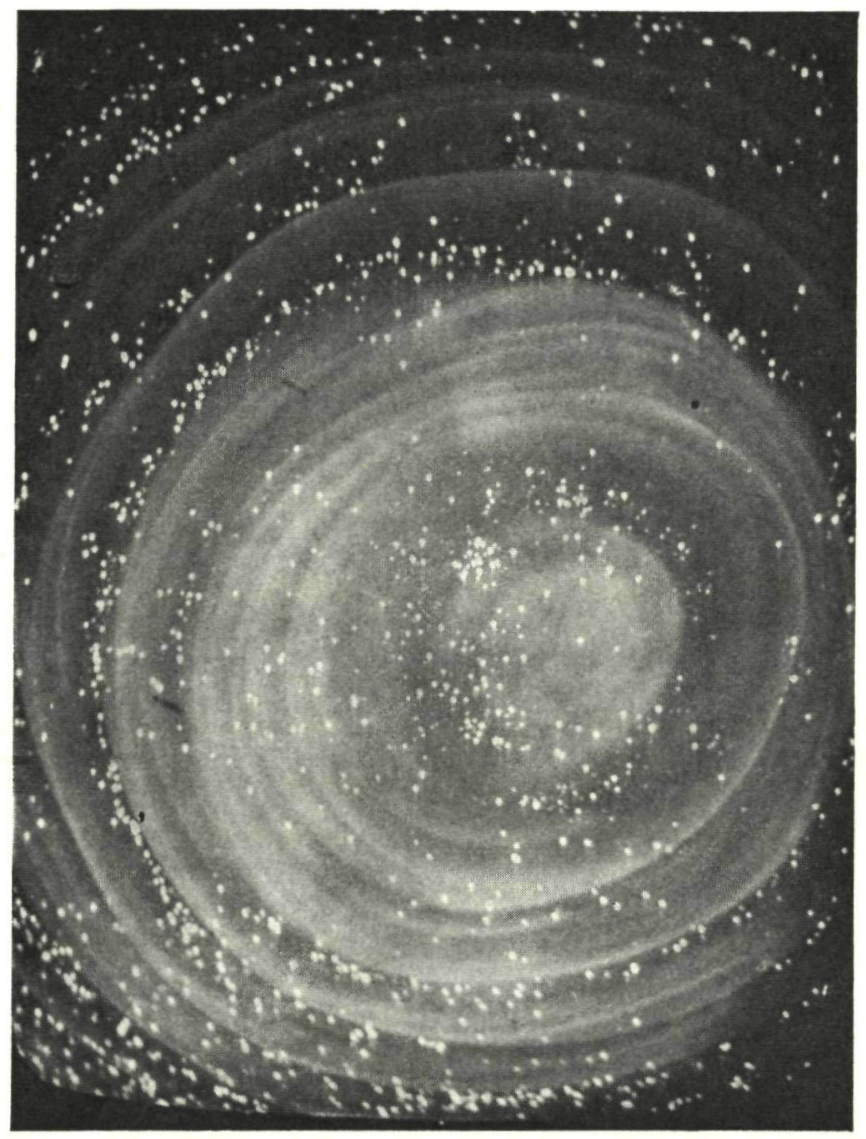

Fig. 3.32. Monitor picture obtained with a target of dislocation-free silicon. Material resistivity: $10 \Omega \mathrm{cm}, n$-type. Target not illuminated. $V_{\mathbf{T}}=10 \mathrm{~V}$. Apart from leaky diode regions a background leakage-current pattern is visible. 
durıng the oxidation and diffusion processes, necessary for diode fabrication (see sec 363 )

These results produce conclusive evidence that microdefects present near or inside the $p-n$ junction of a planar diode cause a strong increase in leakage current of that particular diode

The negative influence of the clusters on diode performance could be elımınated by a strong phosphorus-getter diffusion at about $1050{ }^{\circ} \mathrm{C}$ into the back surface of the silıcon slice which is used as target Such a getter diffusion removes most of the metallic impurities introduced during fabrication of the target ${ }^{3-16{ }^{17}}$ ) Thus indicates that the increased leakage currents are caused by an interaction between the metallic impuritıes and the A-clusters As discussed already in sec 34 , the interaction between A-clusters and, for instance, copper impurities is very strong indeed The A-clusters and stacking faults, from which the metallic ımpurities have been removed, seem to be electrically non-active

\section{REFERENCES}

3-1) F Vieweg-Gutberlet, Solıd-State Electron 12, 731, 1969

3-2) J A M Dikhorf, Solıd-State Electron 1, 202, 1960

3 3) J A M Dikhoff, Philips tech Rev 25, 195, 1963/64

3 4) W C Dash, J appl Phys 27, 1193, 1956

3 5) G H Schwuttke, J electrochem Soc 108, 163, 1961

3-6) L Fiermans and J Vennık, Phys Stat sol 12, 277, 1965

3-7) T F Ciszek, J Crystal Growth 10, 263, 1971

3-8) M G Collet, J electrochem Soc 117, 259, 1970

3-9) C S Fuller and J C Severiens, Phys Rev 96, 21, 1954

3-10) E M Pell, J appl Phys 31, 1675, 1960

3-11) J C Irvin, Bell Sys tech J 41, 387, 1962

3-12) T Takabatake, T Furuga and $Y$ Ueda, Jap J appl Phys 9, 416, 1970

3 13) $M$ H Crowell and $E$ F Labuda, Bell Sys tech J 48, 1481, 1969

3 14) $H$ J Queisser and $P G G$ van Loon, J appl Phys 35, 3067, 1964

3 15) A W Fisher and J A Amick, J electrochem Soc 113, 1054, 1966

3-16) J E Lawrence, Trans AIME 242, 484, 1968

3-17) M Nakamura and T Kato, Jap J appl Phys 7, 512, 1968

3-18) $P$ K Weimer, J V Forgue and $R$ R Goodrich, RCA Rev 12, 306, 1951

3 19) $A$ J R de Kock and P G T Boonen, J electrochem Soc 119, 1241, 1972 


\section{DISCUSSION}

In this chapter we will first discuss the character, size and concentration of the microdefects detected in dislocation-free floating-zone silicon crystals. Later on in this chapter, a model for the formation of these microdefects will be developed which explains all the experimental observations.

\subsection{The character of the grown-in microdefects}

The experimental observations described in chapter 3 showed that a striated distribution of microdefects is present in as-grown dislocation-free floating-zone silicon crystals. These small defects originate from a precipitation process occurring during crystal growth. Essentially, precipitation of two different kinds of point defects can take place: (i) impurities (sec. 1.3.1) and (ii) thermally generated vacancies (sec. 1.3.3.1). As pointed out in sec. 1.3.1, homogeneous precipitation of impurities due to supersaturation will be negligible because of the high purity of the material. However, this does not apply to vacancies. Upon cooling from the melting point the crystal becomes supersaturated with vacancies because the equilibrium vacancy concentration decreases with decreasing temperature (eq. (1.2)). If no vacancy sinks (dislocations) are present, and as long as no new nucleation sites are formed, the excess vacancies can only be annihilated by diffusion to the crystal surface. The following calculation will show that this out-diffusion process is of minor importance. The process can be described by the following differential equation:

$$
D_{\mathbf{v}}\left(\frac{\partial^{2} N_{\mathbf{v}}}{\partial r^{2}}+\frac{1}{r} \frac{\partial N_{\mathbf{v}}}{\partial r}\right)=\frac{\partial N_{\mathbf{v}}}{\partial t} .
$$

The boundary conditions are

$$
\begin{array}{lll}
t=0 & \text { and } \quad 0<r<R \rightarrow N_{\mathrm{v}}=N_{\mathrm{v}}\left(T_{M}\right), \\
t>0 & \text { and } \quad r=R & \rightarrow N_{\mathrm{v}}=0, \\
t \geqslant 0 & \text { and } \quad r=0 & \rightarrow
\end{array}
$$

where $D_{\mathrm{v}}$ is the diffusion coefficient of the vacancies, $N_{\mathrm{v}}$ the vacancy concentration and $R$ the radius of the cylindrical crystal. The solution of this equation is ${ }^{4-1}$ )

$$
\frac{N_{\mathrm{v}}(r, t)}{N_{\mathrm{V}}\left(T_{M}\right)}=\sum_{n=0}^{\infty} A_{n} \exp \left(-\beta_{n}{ }^{2} D_{\mathrm{v}} t / R^{2}\right) . J_{0}\left(\beta_{n} \frac{r}{R}\right)
$$


with

$$
A_{n}=\frac{2}{\beta_{n} J_{1}\left(\beta_{n}\right)} \text {. }
$$

In this expression $J_{0}$ and $J_{1}$ are the zero- and first-order Bessel functions and $\beta_{n}$ are the roots of the equations $J_{0}\left(\beta_{n}\right)=0\left(\beta_{0}=2.405 ; \beta_{1}=5.520 ; \beta_{2}=\right.$ $8.654 ; \beta_{3}=11.792$ ). In order to calculate the radial distribution of vacancies present during growth, let us consider the out-diffusion of vacancies during cooling of a solidified crystal area from, for instance, 1420 to $1000^{\circ} \mathrm{C}$. The axial temperature gradient in a floating-zone crystal is about $300^{\circ} \mathrm{C} / \mathrm{cm}$. With a growth rate of $1 \mathrm{~mm} / \mathrm{min}$ a temperature of $1000{ }^{\circ} \mathrm{C}$ is reached in about 13 min. During cooling $D_{\mathrm{V}}$ continuously decreases. However, because of the low values for the migration energy $E_{\mathrm{V}}{ }^{\mathrm{M}}$, the decrease of $D_{\mathrm{V}}$ is very gradual. For $E_{\mathrm{V}}{ }^{\mathrm{M}}=0 \cdot 18 \mathrm{eV}$ we have $D_{\mathrm{v}}\left(T_{M}\right)=4 \cdot 1 \cdot 10^{-5} \mathrm{~cm}^{2} / \mathrm{s}$ (sec. 1.3.3.2) and $D_{\mathrm{v}}\left(1000^{\circ} \mathrm{C}\right)=2 \cdot 7 \cdot 10^{-5} \mathrm{~cm}^{2} / \mathrm{s}$. Therefore, in a first approximation, we can neglect the temperature dependence of $D_{\mathrm{v}}$. Using an average value for $D_{\mathrm{v}}$ of $3.10^{-5} \mathrm{~cm}^{2} / \mathrm{s}$ we can calculate the radial vacancy profile $N_{\mathrm{v}}(r)$ at $1000^{\circ} \mathrm{C}$. For a crystal with $R=1.25 \mathrm{~cm}$ we obtain the following results:

at the centre of the crystal: $\frac{N_{\mathrm{v}}\left(r=0 ; T=1000^{\circ} \mathrm{C}\right)}{N_{\mathrm{V}}\left(T_{M}\right)}=0.992$

and at $r=1.05 \mathrm{~cm}: \quad \frac{N_{\mathrm{v}}\left(r=1.05 ; T=1000^{\circ} \mathrm{C}\right)}{N_{\mathrm{v}}\left(T_{M}\right)}=0.635$.

In this calculation (4.2) has been approximated by the first four terms of the series. It is clear that the decay in vacancy concentration in the bulk of the crystal during growth proceeds very slowly, and a high supersaturation is built up. The excess vacancies will tend to precipitate in order to return to a stable situation. From this and the foregoing analysis we can conclude that the formation of the microdefects is due to clustering of vacancies.

This conclusion is consistent with the following experimental observations:

(a) The presence of a few dislocations $\left(\approx 1000 \mathrm{~cm}^{-2}\right)$ in the crystal already inhibits the formation of the microdefects. Because vacancies are very mobile in silicon (sec. 1.3.3.2) it can be expected that a relatively low concentration of vacancy sinks in the bulk of the crystal (dislocations) will suffice to annihilate the excess vacancies during cooling. A similar observation has been made for germanium crystals with a low dislocation density ${ }^{4-2,3,4}$ ).

(b) A crystal surface region of about $2 \mathrm{~mm}$ width exhibits a much lower microdefect concentration than the bulk of the crystals (secs 3.2 and 3.3). The surface will act as an effective sink for vacancies. The excess vacancies present 
in the vicinity of the surface partly diffuse out and disappear as is shown by the previous calculation. This results in a relatively low supersaturation with vacancies and the tendency for vacancy clusters to form in this region will therefore remain small.

(c) During high-temperature annealing treatments the microdefects change into stacking faults surrounded by circular dislocation loops (sec. 3.6.2). Although other mechanisms for loop formation also exist, such loops can be formed by the collapse of vacancy clusters, as was first pointed out by Frank ${ }^{4-5}$ ). Similar dislocation loops have since been observed in, for instance, aluminium ${ }^{4-6}$ ) and diamond ${ }^{4-7}$ ).

\subsection{The shape of the vacancy clusters}

The grown-in microdefects formed as a result of the coalescence of vacancies may be present in essentially three different config'urations. For a small number of vacancies per cluster the most reasonable shape is that of a spherical void because of its low surface energy. If a large number of vacancies accumulates on such a spherical void, the diameter will finally reach a critical value above which the void is no longer a stable configuration ${ }^{4-8}$ ). At this critical diameter, which is not known for silicon, the void will collapse, resulting in the formation of the second possible cluster shape, namely an intrinsic stacking fault, surrounded by a dislocation loop (see also fig. 3.27). In this situation the excess free energy due to the dislocation and stacking fault should be less than that of the void. The third possible cluster configuration has been postulated by Hornstra ${ }^{4-9}$ ). This configuration consists of an ordered arrangement of the vacancies in such a way that the dangling bonds of the atoms which are direct neighbours of the vacancies are eliminated by the formation of double bonds. This results in localized regions with a super-lattice structure ("sponge") having an average vacancy concentration of about $8 \%$.

The etching, decoration and annealing techniques used for the study of the vacancy clusters are indirect detection methods and consequently they give little information about the actual shape of the defects. Efforts were therefore made to reveal the defects directly by using an electron microscope in transmission (Philips EM 300). In a number (6) of circular silicon disks with a diameter of $3 \mathrm{~mm}$ and a thickness of $200 \mu \mathrm{m}$, holes were etched with an $\mathrm{HF}-\mathrm{HNO}_{3}$ mixture. Parts of the edge around the holes were transparent to the electron beam (thickness $<2.10^{-5} \mathrm{~cm}$ ). If the voids present in the transparent region are sufficiently large $\left(>10^{-5} \mathrm{~cm}\right)$, they may be revealed by the Möllenstedt ${ }^{4-10}$ ) interference fringes inside the defects, while large dislocation loops will be visible directly. Small voids and small dislocation loops may appear as black dots. The superlattice structure will probably remain undetected.

None of the above-mentioned defect contrasts were detected by electron 
microscopy. This may indicate that the clusters exhibit the "sponge" structure. It is, however, also possible that the defects were not detected because they were etched out before the required thickness of $2.10^{-5} \mathrm{~cm}$ had been reached. This may happen if the size of the strain field of the defects is of the same order as the thickness of the sample or larger (see sec. 4.3). In conclusion it can be said that the exact shape of the clusters is still open to discussion.

\subsection{The size of the vacancy clusters}

The size of the vacancy clusters could not be determined directly because the defects remain undetectable in the transmission electron microscope. Only a rough estimate can be made. Suppose that $50 \%$ of the vacancy concentration present at the melting point $\left(N_{\mathrm{v}}\left(T_{M}\right)\right)$ nucleates in the form of A-clusters while most of the remaining vacancies nucleate in the form of B-clusters. $N_{\mathrm{v}}\left(T_{M}\right)$ is not exactly known (sec. 1.3.3). The theoretical estimates of Seeger and Swanson 4-11) give a lower limit of $1 \cdot 2 \cdot 10^{13} \mathrm{~cm}^{-3}$ and an upper limit of $9.10^{15} \mathrm{~cm}^{-3}$, whereas the extrapolated experimental data seem to point to much higher values $\left(2 \cdot 10^{17}-3 \cdot 10^{19} \mathrm{~cm}^{-3}\right)$. If the theoretically estimated limits for $N_{\mathrm{v}}\left(T_{M}\right)$ are used the following defect sizes are found.

For a crystal with an A-cluster concentration of $10^{6} \mathrm{~cm}^{-3}$ (see sec. 4.4) and if $N_{\mathrm{v}}\left(T_{M}\right)$ is taken equal to the upper limit $9.10^{15} \mathrm{~cm}^{-3}$, the average number of vacancies per A-cluster is $4 \cdot 5.10^{9}$. If the A-clusters have the form of a spherical void, this implies an average diameter of the defects $\left(d_{c}\right)$ of $6.10^{-5} \mathrm{~cm}$. If the voids have collapsed into a single-atomic-layer stacking faults in the (111) planes $\left(7.84 .10^{14} \mathrm{at} / \mathrm{cm}^{2}\right)$, surrounded by circular dislocation loops, we obtain for these loops: $d_{c}=2 \cdot 8 \cdot 10^{-3} \mathrm{~cm}$. If the clusters exhibit the super-lattice structure with a vacancy concentration of $8 \%$ and are spherical in shape, we have $d_{c}=1 \cdot 3 \cdot 10^{-4} \mathrm{~cm}$. Similar calculations can be made using the theoretical lower limit for $N_{\mathrm{v}}\left(T_{M}\right)$. This, together with the preceding calculations, results in the following possible intervals for the $d_{c}$ values:

spherical voids : $6 \cdot 6 \cdot 10^{-6} \mathrm{~cm}<d_{c}<6 \cdot 10^{-5} \mathrm{~cm}$,

circular dislocations: $1 \cdot 0 \cdot 10^{-4} \mathrm{~cm}<d_{c}<2 \cdot 8 \cdot 10^{-3} \mathrm{~cm}$,

spherical "sponges": $1 \cdot 4 \cdot 10^{-5} \mathrm{~cm}<d_{c}<1 \cdot 3 \cdot 10^{-4} \mathrm{~cm}$.

If the experimentally determined values for $N_{\mathrm{v}}\left(T_{M}\right)$ referred to above are taken, we obtain for

spherical voids $: 1 \cdot 6 \cdot 10^{-4} \mathrm{~cm}<d_{c}<8 \cdot 10^{-4} \mathrm{~cm}$,

circular dislocations: $1 \cdot 2 \cdot 10^{-2} \mathrm{~cm}<d_{c}<7 \cdot 8 \cdot 10^{-2} \mathrm{~cm}$,

spherical "sponges": $3 \cdot 6 \cdot 10^{-4} \mathrm{~cm}<d_{c}<1 \cdot 9 \cdot 10^{-3} \mathrm{~cm}$.

The vacancy clusters do not cause contrast in X-ray transmission topog- 
raphy (sec. 3.3). This indicates that the diameter of the strain field around clusters having the character of a void or stacking fault is of the order of $10^{-4} \mathrm{~cm}$ or smaller (sec. 2.5). The $d_{c}$ values calculated for the void structures on the basis of the theoretically estimated vacancy concentrations therefore seem to be reasonable, while those based on the experimental data may be too large. We further notice that the $d_{c}$ values calculated for the dislocation loops are too high except for the one calculated for $N_{\mathrm{v}}\left(T_{M}\right)=1 \cdot 2 \cdot 10^{13} \mathrm{~cm}^{-3}$. This indicates that either the defects are not present in the form of loops or $N_{\mathrm{V}}\left(T_{M}\right) \leqslant 10^{13} \mathrm{~cm}^{-3}$.

Because the sponge defects will only cause minor distortions of the silicon lattice, the large dimensions calculated for this defect structure are probably not inconsistent with the absence of defect contrast in X-ray topography.

\subsection{Determination of the concentration of vacancy clusters}

It was observed by means of preferential etching (sec. 3.2) and copper decoration (sec. 3.3) that two types of vacancy clusters, differing in size, were present. From carbon replicas of preferentially etched surfaces (fig. 3.5) it was established that the concentration of the small defects (B-clusters) is greater than that of the larger defects. The concentration of the A-clusters $\left(N_{\mathrm{A}}\right)$ is readily determined by copper or lithium decoration in combination with $\mathrm{X}$-ray transmission topography. Concentrations between $5.10^{5}$ and $10^{7} \mathrm{~cm}^{-3}$ were measured in the various crystals. Exact determination of the B-cluster concentration $\left(N_{\mathrm{B}}\right)$ is more difficult. Although these defects are present throughout the crystal, copper decoration only reveals B-clusters present in regions containing few A-clusters (surface and cone region (sec. 3.3)). This must be due to marked interaction between copper atoms and A-clusters in the bulk of the crystal, which dominates the precipitation kinetics, so that not much copper precipitates on the B-clusters. This causes the B-clusters to remain invisible.

The A-cluster concentration in the argon-grown crystal shown in figs 3.2 and 3.11 was $2.10^{6} \mathrm{~cm}^{-3}$. In this particular crystal the B-clusters gave rise after preferential etching to the formation of tiny pits which were visible in the optical microscope (fig. 3.4). From the etch-pit distribution it was established (sec. 3.2) that $N_{\mathrm{B}} \approx 15 N_{\mathrm{A}}$ and consequently $N_{\mathrm{B}}=3.10^{7} \mathrm{~cm}^{-3}$. Usually, however, the etch pits formed on the B-clusters can only be detected with the aid of carbon replicas, making accurate determination of $N_{\mathbf{B}}$ impossible.

The concentration of the B-clusters can also be determined from the electrical behaviour of Li during its precipitation process, for, although the B-clusters do not become visible in this decoration process, it is reasonable to expect that the $\mathrm{Li}$ atoms will be captured by the A-clusters as well as by the smaller B-clusters. As described in sec. 3.5, exclusively heterogeneous precipitation on grown-in defects is detected after lithium diffusions at $T_{D} \leqslant 400{ }^{\circ} \mathrm{C}$.

In dislocation-free material containing only $\mathrm{A}$ - and B-clusters we will there- 
fore have: $N_{\mathrm{p}}=N_{\mathrm{A}}+N_{\mathrm{B}}$, where $N_{\mathrm{p}}$ is the concentration of lithium nucleation sites. The value of $N_{\mathrm{p}}$ and consequently the total cluster concentration can be calculated as follows. The precipitation of lithium in silicon is known to be diffusion-limited ${ }^{4-12}$ ). Consequently, the precipitation theory of Ham $^{4-13}$ ) will apply. For the unprecipitated amount of lithium at annealing time $t\left(C_{\mathrm{L} 1}(t)\right)$ we have

$$
\frac{C_{\mathrm{L} 1}(t)-C_{\mathrm{L} 1}(\infty)}{C_{\mathrm{Li}}(0)-C_{\mathrm{L} 1}(\infty)}=\exp -\left(\frac{t}{\tau}\right)^{n},
$$

where $C_{\mathrm{L}}(0)$ and $C_{\mathrm{LI}}(\infty)$ are the solute contents at $t=0$ and $t=\infty$ respectively, $\tau$ is the precipitation relaxation time and $n$ is a number. When $C_{\mathrm{L} I}(t)>0.5 C_{\mathrm{Li}}(0)$ the value of $n$ depends on the shape of the precipitates, whereas for $C_{\mathrm{Li}}(t)<0.5 C_{\mathrm{LI}}(0)$ the value of $n$ is equal to 1 , leading to simple exponential decay ${ }^{*}$ ). The relaxation time $\tau$ can be determined from the linear part of the precipitation curve (fig. 3.20). $\mathrm{Ham}^{4-13}$ ) has derived the following relationship between $\tau$ and $N_{\mathrm{p}}$ :

$$
\frac{1}{\tau}=\left(48 \pi^{2} C_{\mathrm{Li}}(0) v\right)^{1 / 3} F(e) D_{\mathrm{L} 1} N_{\mathrm{p}}^{2 / 3},
$$

where $v$ is the precipitate volume per solute atom $(\mathrm{Li})$ and $D_{\mathrm{L} \text { t }}$ the diffusion coefficient of lithium at the annealing temperature $\left(160^{\circ} \mathrm{C} ; D_{\mathrm{L}_{1}}=4 \cdot 8 \cdot 10^{-11}\right.$ $\left.\mathrm{cm}^{2} / \mathrm{s}\right)$. The geometrical factor $F(e)$ is a function of the eccentricity $e$ of the precipitates formed. The eccentricity of spheroidal precipitates is defined by $e=\left(1 / a_{1}\right)\left(a_{1}{ }^{2}-b_{1}{ }^{2}\right)^{1 / 2}$, where $a_{1}$ and $b_{1}$ are the large and small half-axes of the precipitate. $F(e)$ is approximately equal to $\left(1-e^{2}\right)^{-1 / 6}$. For spherical precipitates $(e=0)$ we have $F(e)=1$, whereas this factor very slowly increases with increasing eccentricity. With regard to the value of $e$ that we have used in our calculations, we note that infrared microscopy has revealed that the lithium precipitates have the shape of flat circular platelets. Precipitates of similar shape were found by Ferman ${ }^{4-12}$ ), who estimated the eccentricity of these precipitates to be 0.99 . This value has also been used for our crystals, giving $F(e)=1.92$. As regards $v$, we used the value of Pell ${ }^{4-14}$ ). He related transmission electronmicroscopic determinations of the size and concentration of lithium precipitates in silicon samples to the total amount of precipitated lithium, measured electrically, and estimated $v$ to be $3 \cdot 2 \cdot 10^{-22} \mathrm{~cm}^{3}$. The value of $N_{\mathrm{p}}$ can be determined from the curves shown in fig. 3.20 in combination with eqs (4.3) and (4.4).

*) It has been found 4-12), however, that the region of simple exponentlal decay extends to much larger values of $C_{\mathrm{L},}(t)$. This has been attributed by Ferman et al. ${ }^{4-12}$ ) to an inhomogeneous distribution of the $\mathrm{L}_{1}$ donors around the nucleation sites at $t=0$. 
For the argon-grown crystal shown in figs 3.11 and 3.19 we obtain $N_{\mathrm{p}}=N_{\mathrm{A}}+N_{\mathrm{B}}=5-8.10^{7} \mathrm{~cm}^{-3}$. Since $N_{\mathrm{A}}=2.10^{6} \mathrm{~cm}^{3}$, we have $N_{\mathrm{B}} \approx 5-8 \cdot 10^{7} \mathrm{~cm}^{-3}$. This value ${ }^{*}$ ) agrees well with that estimated from the etch-pit pattern (fig. 3.4). Although eqs (4.3) and (4.4) are derived for a uniform distribution of nucleation sites, the striated cluster distribution apparently has no significant influence on the applicability of these equations (see also Morin and Reiss $\left.{ }^{4-15}\right)$ ).

The vacuum-grown crystal shown in fig. 3.14 contained an A-cluster density of $7.10^{5} \mathrm{~cm}^{-3}$. From fig. 3.20 we obtain $N_{\mathrm{p}}=N_{\mathrm{A}}+N_{\mathrm{B}} \approx N_{\mathbf{B}}=2 \cdot 10^{7}$ $\mathrm{cm}^{-3}$.

A third method of determining $N_{\mathrm{A}}$ and $N_{\mathrm{B}}$ is based on the behaviour of the vacancy clusters during annealing under pure conditions at elevated temperatures (sec. 3.6.2). Both A- and B-clusters change into stacking faults, which cause direct-image contrast in X-ray transmission topographs (fig. 3.26). However, the relatively high concentration of B-clusters causes an overlap of the images on the topograph, making the concentration determination less reliable. For the crystal shown in fig. $3.26(a)$ it was estimated that $N_{\mathrm{B}}>10^{7}$ $\mathrm{cm}^{-3}$.

Summarizing we may say that $N_{\mathrm{A}}$ varies between $5.10^{5}$ and $10^{7} \mathrm{~cm}^{-3}$, while $N_{\mathbf{B}}$ amounts to $10^{7}-10^{8} \mathrm{~cm}^{-3}$.

\subsection{The nature of the vacancy-nucleation sites: homogeneous versus hetero- geneous formation}

Essentially nuclei for condensation of excess point defects may be formed by a homogeneous (vacancy-vacancy) or a heterogeneous (vacancy-impurity) nucleation process. Before we can develop a more or less detailed model for the vacancy-cluster formation in silicon, we have therefore to decide which of these two processes is dominant. If we assume a homogeneous nucleation process to be operative, the nuclei and consequently the vacancies must be present in the same striated pattern as that of the clusters. This can only be understood if we assume that the vacancy concentration introduced during solidification is growth-rate-dependent as indicated earlier by Plaskett ${ }^{4-16}$ ).

Equation (1.1), which describes the growth-rate-dependent incorporation of impurities into the crystal during growth from the melt, cannot be used for point defects such as vacancies, for two reasons: (1) an equilibrium distribution coefficient for vacancies is not easily defined and (2) diffusion in the solid state was neglected in the derivation of eq. (1.1). This is not permissible in the case of very mobile point defects. Webb ${ }^{4-17}$ ), however, has developed a general theory for the trapping of non-equilibrium concentrations of vacan-

*) It should be noted, however, that the calculated value of $N_{p}$ may be too high because no correction has been made for the (unknown) number of lithium donors lost by outdiffusion to the sample surface. 
cies during crystal growth, which offers the possibility to judge whether homogeneous nucleation is likely to occur. In this theory the introduction of a distribution coefficient is avoided, while solid-state diffusion is taken into account. The growth interface, representing the transition region where the melt structure changes into the single-crystal lattice structure, will be diffuse. The vacancy concentration $N_{\mathrm{v}}{ }^{\mathrm{s}}$ in the outermost atomic layer of this diffuse interface will te higher than the equilibrium bulk concentration at the melting point, $N_{\mathrm{v}}\left(T_{M}\right)$. The number of excess vacancies $A N_{\mathrm{v}}$ trapped in the bulk of the crystal depends on the speed with which the outermost layer is covered by successive-layer growth and the up-hill diffusion of the vacancies towards the growth interface. Assuming the thickness $d$ of the diffuse interface to be a few atomic layers only, the following expression could be derived:

$$
1 N_{\mathrm{v}}=\frac{N_{\mathrm{v}}{ }^{\prime}-N_{\mathrm{v}}\left(T_{M}\right)}{1+D_{\mathrm{v}}\left(T_{M}\right) / V_{\mathrm{y}} d}, .
$$

where $D_{\mathrm{v}}\left(T_{M}\right)$ is the bulk diffusion coefficient of vacancies at the melting point and $V_{g}$ the crystal-growth rate. As discussed in sec. 1.3.3.2, the diffusion coefficient of vacancies in the odd-electron charge state at $T=T_{M}$ is $1.4 .10^{-5}$ $\mathrm{cm}^{2} / \mathrm{s}$, and $4 \cdot 1 \cdot 10^{-5} \mathrm{~cm}^{2} / \mathrm{s}$ for the doubly negatively charged vacancy. If we take $d$ equal to $5 \cdot 10^{-8} \mathrm{~cm}$, which means a diffuse interface extending over two atomic layers, we have for a crystal grown at $V_{q}=3 \mathrm{~mm} / \mathrm{min}$ :

$$
\mathcal{A} N_{\mathrm{v}}=\left[N_{\mathrm{v}}{ }^{\mathrm{s}}-N_{\mathrm{v}}\left(T_{M}\right)\right]\left(5 \cdot 6.10^{4}\right)^{-1} \text { for } E_{\mathrm{v}}{ }^{\mathrm{M}}=0.33 \mathrm{eV}
$$

and

$$
A N_{\mathrm{v}}=\left[N_{\mathrm{v}}{ }^{\mathrm{s}}-N_{\mathrm{v}}\left(T_{M}\right)\right]\left(1 \cdot 6.10^{5}\right)^{-1} \text { for } E_{\mathrm{v}}{ }^{\mathrm{M}}=0.18 \mathrm{eV} .
$$

It is reasonable to expect that trapping of at least $10 \%$ excess vacancies is required for the formation of a sharply defined striated pattern of vacancy clusters: $N_{\mathrm{v}} \geqslant 0.1 N_{\mathrm{v}}\left(T_{M}\right)$. This requires

$$
N_{\mathrm{v}}^{\mathrm{s}} / N_{\mathrm{v}}\left(T_{M}\right)>5 \cdot 6 \cdot 10^{3} \text { for } E_{\mathrm{V}}{ }^{\mathrm{M}}=0 \cdot 33 \mathrm{eV}
$$

and

$$
N_{\mathrm{V}}^{\mathrm{s}} / N_{\mathrm{v}}\left(T_{M}\right)>1 \cdot 6 \cdot 10^{4} \text { for } E_{\mathrm{V}}{ }^{\mathrm{M}}=0.18 \mathrm{eV},
$$

which means that the vacancy concentration in the outermost layer of the interface has to be higher than $N_{\mathrm{v}}\left(T_{M}\right)$ by about four orders of magnitude. If $N_{\mathrm{v}}\left(T_{\mathrm{M}}\right)>10^{16} \mathrm{~cm}^{-3}$, this requires unreasonably high values for $N_{\mathrm{v}}{ }^{\mathrm{s}}$, but for the theoretically calculated values of $N_{\mathrm{v}}$, i.e. $N_{\mathrm{v}}\left(T_{M}\right)<10^{16} \mathrm{~cm}^{-3}$, trapping of excess vacancies may still be possible. Consequently, the possibility of vacancy striations is still not excluded.

The concentration of excess vacancies trapped at the solid-liquid interface has to be compared with the excess vacancies originating from the cooling of the crystal after solidification. According to eq. (1.2) $N_{\mathrm{v}}(T)$ decreases exponen- 
tially with temperature. For $E_{\mathrm{V}}{ }^{\mathrm{F}}=4.5 \mathrm{eV}$ (sec. 1.3.3.1) the solidified area already contains a $10 \%$ supersaturation of vacancies after cooling over $7{ }^{\circ} \mathrm{C}$. If $E_{\mathrm{V}}{ }^{\mathrm{F}}=2.3 \mathrm{eV}$ (sec. 1.3.3.1), this occurs after cooling over $11{ }^{\circ} \mathrm{C}$. The axial temperature gradient near the solid-liquid interface in a floating-zone crystal is about $300{ }^{\circ} \mathrm{C} / \mathrm{cm}$. Consequently, a supersaturation of $10 \%$ is achieved about $2 \cdot 3 \cdot 10^{-2}$ to $3 \cdot 6 \cdot 10^{-2} \mathrm{~cm}$ behind the interface. With $V_{g}=3 \mathrm{~mm} / \mathrm{min}$ this takes only 5 to $7 \mathrm{~s}$. Thus, even if $10 \%$ excess vacancies have been trapped, the total supersaturation with vacancies is entirely determined by the cooling of the crystal within $10 \mathrm{~s}$ after solidification. Furthermore, if a striated distribution of vacancies is introduced during growth due to the periodic variation in $V_{g}$ (eq. (4.5)), bulk diffusion will rapidly reduce the periodic variation in $N_{\mathrm{v}}$. This will result in a more uniform vacancy distribution. In the case of homogeneous nucleation of vacancies a more uniform vacancy distribution is inconsistent with the striated cluster distribution detected.

Although the considerations mentioned above do not completely exclude the possibility of homogeneous nucleation, it is assumed that this type of nucleation process is of minor importance. The following experimental observations support this assumption:

(a) When the growth rate is increased from 1 to $3 \mathrm{~mm} / \mathrm{min}$ (figs 3.10 and 3.11 ), the other growth parameters being kept constant, no increase in the concentration of A-clusters is observed. This indicates that the vacancy concentration is not noticeably influenced by variations in $V_{g}$.

(b) $N_{\mathrm{v}}{ }^{\mathrm{s}}$ can be expected to be orientation-dependent ${ }^{4-17}$ ). Nevertheless, equal concentrations of vacancy clusters were found in crystals grown at $3 \mathrm{~mm} / \mathrm{min}$ in either the $\langle 111\rangle$ or the $\langle 100\rangle$ direction (figs 3.11 and 3.12 ).

A model for heterogeneous nucleation of vacancies in dislocation-free silicon will be presented in the next section.

\subsection{The model}

The nucleation model that will now be discussed is based on the assumption that during cooling of the as-grown crystal various types of stable vacancy-oxygen complexes are formed in a lattice supersaturated with vacancies. Some of the vacancy-oxygen complexes are sufficiently large to act as nuclei for the elimination of excess vacancies:

$$
m \mathrm{~V}+n \mathrm{O}_{-}^{-} V_{m} \mathrm{O}_{n} \stackrel{\text { vacancies }}{\rightarrow} \text { cluster. }
$$

This model originates from the following considerations. As discussed in sec. 4.5, we assume that trapping of excess vacancies at the solid-liquid interface is negligible $\left(\Lambda N_{\mathrm{v}} \approx 0\right)$. This implies that the distribution of vacancies in the bulk of the crystal is uniform. The striated cluster distribution therefore indicates that impurities with a distribution coefficient differing from unity play 
an essential part in the process of nucleus formation. Because of the high purity, homogeneous nucleation of impurities due to supersaturation will not occur (sec. 1.3.1), i.e. impurity precipitates which may act as nuclei for excess vacancies, are not formed. However, it is known that associates of impurity atoms and vacancies such as, for instance, vacancy-oxygen complexes can form in the silicon lattice (sec. 1.3.3.3). As established by Corbett et al. ${ }^{4-18}$ ) vacancy-oxygen associates can be formed in different modifications such as the $\mathrm{V}-\mathrm{O}$ pair and the $\mathrm{VO}_{2}$ and $\mathrm{VO}_{3}$ complexes. Because of this tendency for vacancy-oxygen association and because oxygen is one of the dominant impurities left in F.Z. silicon it is reasonable to assume that oxygen plays a part in the formation of nuclei.

In our model the following process sequence during growth is expected. On cooling, the crystal becomes highly supersaturated with vacancies (sec. 4.1). Simultaneously with the occurrence of the supersaturation with vacancies various types of vacancy-oxygen complexes are formed. In general these complexes consist of $m$ vacancies and $n$ oxygen atoms with $m, n \geqslant 1$. Their formation can be described by the following set of reactions:

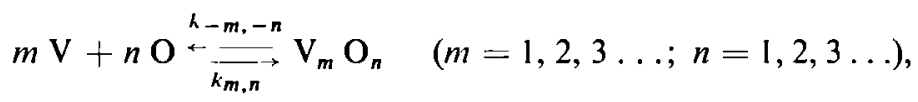

where $k_{m, n}$ and $k_{-m,-n}$ are the rate constants. Because of the limited time available for complex formation during cooling, only complexes having small values of $m$ and $n$ may be present in equilibrium with the existing vacancy and oxygen concentrations. The concentration of the larger complexes will remain below their equilibrium values because they are formed from the smaller complexes. We note, however, that the generation rate of complexes containing $m$ vacancies and $n$ oxygen atoms will be proportional to $\left[N_{\mathrm{v}}\right]^{m}\left[N_{\mathrm{ox}}\right]^{n}$. In addition we shall most probably have that the concentration of $\mathrm{V}_{m} \mathrm{O}_{n}$ complexes $\left(N_{\mathrm{V}_{m} \mathrm{O}_{n}}\right)$ is smaller than the concentration of $\mathrm{V}_{m^{\prime}} \mathrm{O}_{n^{\prime}}$ complexes $\left(N_{\mathrm{V}_{m^{\prime}} \mathrm{O}_{n^{\prime}}}\right)$ if $m>m^{\prime}$ and (or) $n>n^{\prime}$, because the smaller complexes will exhibit higher stability.

In our model we assume that some types of $\mathrm{V}_{m} \mathrm{O}_{n}$ complexes are able to act as nuclei for the elimination of excess vacancies, resulting in the formation of vacancy clusters. The condition for occurrence of this condensation process is, of course, that the total free energy is lowered. From existing nucleation theories which, however, are only valid for homogeneous nucleation, it is known that prior to vacancy condensation, vacancy associates have to be formed, which must exceed a certain critical size before further growth into clusters is possible. The reason for this is that the change in total free energy $(\Lambda G)$ due to the formation of a vacancy associate consists of two terms which counteract each other, viz.

$$
\Delta G=4 \pi r^{2} \sigma+\left(\frac{1}{3} \pi r^{3}\right) \Delta G_{\mathrm{V}},
$$


where

$$
\Delta G_{\mathbf{V}}=-\frac{k T}{v} \ln \frac{N_{\mathrm{v}}}{N_{\mathrm{v}} \text { (eq.) }}
$$

and $r$ is the radius of the vacancy associate which is assumed to be spherical, $\sigma$ the interfacial energy per $\mathrm{cm}^{2}$ which equals the surface tension, $\Delta G_{\mathrm{V}}$ the volume free-energy change per added vacancy, $v$ the atomic volume, $N_{\mathrm{V}}$ the existing vacancy concentration and $N_{\mathrm{V}}$ (eq.) the equilibrium vacancy concentration. Under conditions of vacancy supersaturation $\left(N_{\mathrm{V}} / N_{\mathrm{V}}\right.$ (eq. $\left.)>1\right) \Delta G_{\mathrm{V}}$ will be negative. $\Delta G$ is plotted versus $r$ in fig. 4.1. The function $\Delta G(r)$ exhibits a maximum $\left(\Delta G_{c}\right)$ at $r=r_{c}$, for which we derive:

$$
r_{c}=\frac{2 \sigma v}{k T \ln \left[N_{\mathrm{v}} / N_{\mathrm{v}}(\text { eq. })\right]} .
$$

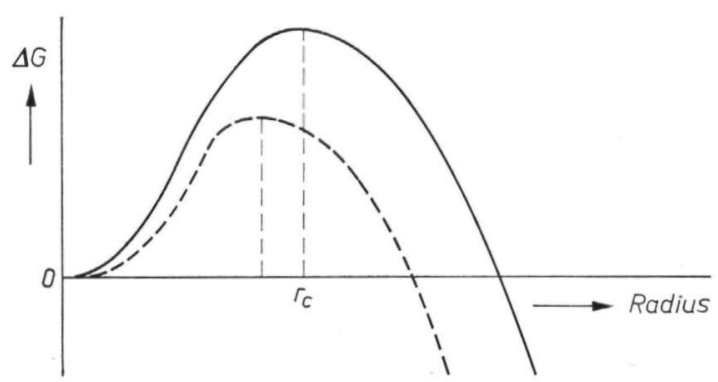

Fig. 4.1. The change of the total free energy due to a homogeneously formed vacancy associate as a function of radius. The radius of the critical nucleus is called $r_{c}$. The dashed curve applies to a higher supersaturation of vacancies.

Vacancy associates with a radius larger than the critical radius $r_{c}$ will act as nuclei for the condensation of vacancies. From eq. (4.12) it can be seen that the critical radius decreases with increasing supersaturation (dashed curve in fig. 4.1).

Returning to our heterogeneous nucleation model we can say that vacancy-oxygen complexes with a radius about equal to $r_{c}$ will also be able to act as critical nuclei for vacancy condensation.

We previously assumed (sec. 4.5) that the homogeneous process is of minor importance, but we are now able to calculate under what condition this assumption is valid. The equilibrium concentration $\left(N_{c}\right)$ of homogeneously formed critical nuclei is given by

$$
N_{c}=N_{\mathrm{v}} \exp \left(-\Delta G_{c} / k T\right) .
$$

Homogeneous nucleation is negligible if this $N_{c}$ is small compared with the 
experimentally determined concentration of vacancy clusters:

$$
N_{c}<10^{6} \mathrm{~cm}^{-3} \text {. }
$$

Substituting (4.11) and (4.12) in (4.10) we obtain for the maximum total freeenergy change:

$$
A G_{c}=\frac{16 \pi \sigma^{3} v^{2}}{\left.3\left\{k T \ln \left[N_{\mathrm{v}} / N_{\mathrm{v}} \text { (eq. }\right)\right]\right\}^{2}} .
$$

Taking a value of $10^{15} \mathrm{~cm}^{-3}$ for $N_{\mathrm{v}}$ (sec. 1.3.3.1) and for the crystal temperature at which vacancy condensation takes place a value of, say, $1500 \mathrm{~K}$, we obtain by substitution of (4.13) and (4.15) in (4.14):

$$
A G_{c}>2 \cdot 7 \mathrm{eV} \text {. }
$$

If we take the formation energy of a vacancy as being $2.3 \mathrm{eV}$ (sec. 1.3.3.1), substitution of (4.15) in (4.16) shows that heterogeneous nucleation of vacancies is dominant when the following condition is fulfilled:

$$
\sigma>478 \mathrm{dyn} / \mathrm{cm} \text {. }
$$

The critical value of $478 \mathrm{dyn} / \mathrm{cm}$ is only slightly changed if other reasonable values for $E_{\mathrm{V}}{ }^{\mathrm{F}}, T$ and $N_{\mathrm{v}}$ are used. Experimental values for the surface tension of solid silicon at high temperatures are not available. Jaccodine ${ }^{4-19}$ ), however, measured the surface tension of silicon at room temperature as well as at liquid-nitrogen temperature. Under both temperature conditions a value of about $1230 \mathrm{dyn} / \mathrm{cm}$ was found for (111) surfaces, while the values for (110) and (100) surfaces were estimated to be 1510 and $2130 \mathrm{dyn} / \mathrm{cm}$, respectively. The surface tension of molten silicon at $1420^{\circ} \mathrm{C}$ is $720 \mathrm{dyn} / \mathrm{cm}$. From these data it is concluded that condition (4.17) for the dominance of heterogeneous nucleation is very reasonable and consequently it supports the previously made assumption that homogeneous nucleation is of minor importance in our crystals.

Now that we have shown it to be reasonable to postulate that vacancy-oxygen complexes act as condensation nuclei, we will look at the condensation process in more detail. During cooling of the as-grown crystal the first type of critical complexes (e.g. $\mathrm{V}_{m} \mathrm{O}_{n}$ ) will be formed at a certain critical temperature $T_{c}$ (fig. 4.2). Consequently, precipitation of vacancies on these nuclei takes place at $T<T_{c}$. It is also possible that a fraction of these complexes was already formed at temperatures above $T_{c}$, but that they did not act as nucleation sites immediately after their formation because the required vacancy supersaturation had not yet been attained. During cooling of the crystal below $T_{c}$ the generation rate of the $\mathrm{V}_{m} \mathrm{O}_{n}$ complex will increase at first because the stability of 


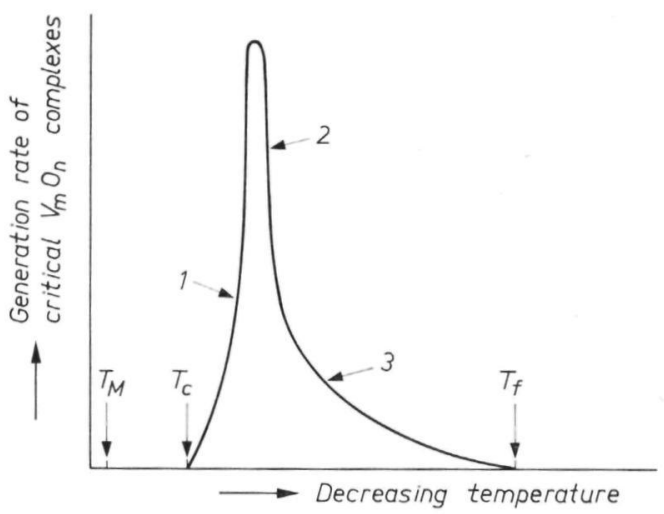

Fig. 4.2. Generation rate of critical $V_{m} O_{n}$ complexes versus temperature during crystal growth. Slope 1: increasing rate due to increasing complex stability. Slope 2: decreasing rate due to the reduction of the vacancy supersaturation. Slope 3 : decreasing rate due to the decrease in $N_{\mathrm{V}}(T)$ and $D_{\text {ox }}(T)$.

these complexes will increase with decreasing temperature. However, owing to the cluster formation, the concentration of excess vacancies will rapidly decrease, soon resulting in a pronounced decrease of the complex-generation rate (fig. 4.2). If $E_{\mathrm{V}}{ }^{\mathrm{F}}=2 \cdot 3 \mathrm{eV}$ (sec. 1.3.3.1) and, for instance, $T_{c}=1200{ }^{\circ} \mathrm{C}$, the vacancy concentration will decrease by one order of magnitude. Furthermore, because of the high activation energy for oxygen diffusion $\left.(2 \cdot 56-3 \cdot 5 \mathrm{eV})^{4-20,21}\right)$, $D_{\text {ox }}$ will decrease steeply with decreasing temperature. This will lower the complex-generation rate still further. Because of this the formation of additional complexes of the same kind $\left(\mathrm{V}_{m} \mathrm{O}_{n}\right)$ will only be possible in a relatively small temperature interval $T_{c}-T_{f}$.

Although for $T<T_{c}$ internal vacancy sinks are present in the form of vacancy clusters, a supersaturation with vacancies will persist during cooling because the vacancy precipitation process will be diffusion-limited. Because of the gradual decrease of $D_{\mathrm{v}}$ during cooling and the constant number of clusters the process whereby excess vacancies are captured slows down. This will finally result in an increase of the relative supersaturation. Another type of vacancy-oxygen associate (e.g. the $\mathrm{V}_{m^{\prime}} \mathrm{O}_{n^{\prime}}$ complex) can therefore be expected to start acting as a new critical nucleus at $T<T_{c^{\prime}}$. These associates will also soon be changed into vacancy clusters. For reasons similar to those mentioned above, additional $\mathrm{V}_{m^{\prime}} \mathrm{O}_{n^{\prime}}$ complexes will only be formed in a second short temperature interval $T_{c^{\prime}}-T_{f^{\prime}}$. Similar precipitation processes may occur for $T<T_{c^{\prime \prime}} \ldots$, etc.

In the next section we will discuss how the experimental results, already described in chapter 3, can be explained with the model for heterogeneous nucleation of vacancies. 


\subsection{Explanation of the experimental results}

The most essential experimental results described in chapter 3 are summarized below:

(a) Two types of clusters (A, B) are formed, which differ in size and concentration.

(b) The vacancy clusters are formed in a sharply defined striated pattern. These striations exhibit randomly distributed interruptions.

(c) Only B-clusters are present in the crystal surface and the upper cone region of crystals grown in argon, whereas these regions are perfect for vacuumgrown material. The dislocation-free region I of the thin neck usually contains no clusters, while the region IV (fig. 3.7(c)) exhibits an increase in cluster concentration.

(d) The vacancy-cluster concentration generally exhibits a minimum in the central region of the crystal.

$\operatorname{Re}(a)$. During cooling of the as-grown crystal various types of vacancy-oxygen complexes act as nucleation sites for vacancies. This results in the formation of various types of vacancy clusters. The first cluster type is formed at temperatures below $T_{c}$ by vacancy condensation on the $\mathrm{V}_{m} \mathrm{O}_{n}$ complexes. These clusters will become relatively large because a high absolute concentration of excess vacancies is captured on a small number of $\mathrm{V}_{m} \mathrm{O}_{n}$ complexes (sec. 4.6). The vacancy clusters formed at the lower temperatures $\left(T_{c^{\prime}}, T_{c^{\prime \prime}} \ldots\right.$ ) will remain smaller, on the one hand because their concentration will be higher, and on the other because the concentration of excess vacancies is smaller due to the preceding condensation on the $\mathrm{V}_{m} \mathrm{O}_{n}$ complexes. This accounts for the presence of large A-clusters and small B-clusters. All the experimental results indicate that only these two categories of microdefects are formed. This means that not more than two types of vacancy-oxygen complexes capable of acting as critical nuclei are formed during growth.

The values of $N_{\mathrm{B}}$ determined are larger than $N_{\mathrm{A}}$ by one to two orders of magnitude. Obviously, the concentration of nuclei for B-clusters $\left(\mathrm{V}_{m^{\prime}} \mathrm{O}_{n^{\prime}}\right.$ complexes) is relatively large. This can be expected because more time is available for their formation $\left(T_{c^{\prime}}<T_{c}\right)$.

Because the formation of all A-clusters starts in a relatively small temperature interval $\left(T_{c}-T_{f}\right)$, the clusters may be expected to exhibit only a small spread in size. This is confirmed by the fact that the copper and lithium precipitates formed on them after decoration are all about the same size (see fig. 3.9).

The size of the B-clusters will be largest in those regions where the A-clusters are absent, because they will capture more vacancies there. This is confirmed by the preferential-etching results obtained with the argon-grown crystal shown in fig. 3.4. In spite of the loss of vacancies near the surface due to out-diffusion 
(eq. (4.1)), the B-cluster etch pits were largest in the surface and upper cone region.

One other phenomenon we have to comment on is why there is only a relatively small variation (a factor 20) in the concentration of A-clusters between all the crystals investigated, which were grown under different conditions. For instance, the oxygen concentration measured in these crystals ranged from $2.10^{16} \mathrm{~cm}^{-3}$ to values below $5 \cdot 10^{15} \mathrm{~cm}^{-3}$. If it is assumed that the nuclei for A-clusters contain, say, 4 oxygen atoms, differences in $N_{\mathrm{A}}$ of about a factor 256 would be expected. Furthermore, differences in growth rate may influence the value of $N_{\mathrm{A}}$ because the time available for the formation of nuclei depends on the cooling rate of the crystal. We shall first elucidate the influence of the oxygen concentration on the generation of $\mathrm{V}_{m} \mathrm{O}_{n}$ complexes and then discuss the influence of the growth rate on nucleus formation. As regards the oxygen concentration, we note that, if only a fraction of the available vacancies and oxygen atoms is bound in the form of complexes, the generation rate of $\mathrm{V}_{m} \mathrm{O}_{n}$ complexes is proportional to $\left[N_{\mathrm{V}}\right]^{m}\left[N_{\mathrm{ox}}\right]^{n}$. This does not imply that a reduction of $N_{\text {ox }}$ by a factor $F$ would cause a reduction in complex concentration by a factor $F^{n}$ as we have also to take the value of $N_{\mathrm{V}}$ into account. $N_{\mathrm{V}}$ will remain constant as long as no vacancy precipitation has occurred. When the initial generation rate of complexes is low $\left(N_{\text {ox }}\right.$ low), more time is required to create sufficient vacancy nucleation sites to eliminate the high supersaturation of the vacancies, and supersaturation therefore persists longer. Consequently, a reasonable generation of $\mathrm{V}_{m} \mathrm{O}_{n}$ complexes will take place during a longer time interval (temperature interval) compared with larger values of $N_{\text {ox }}$ (see fig. 4.3). Furthermore, the crystals with the lowest $N_{\text {ox }}$ are generally obtained by crystal growth in a high vacuum (sec. 1.3.1). These crystals exhibit a lower cooling rate than those grown in argon. Hence they stay longer in the critical

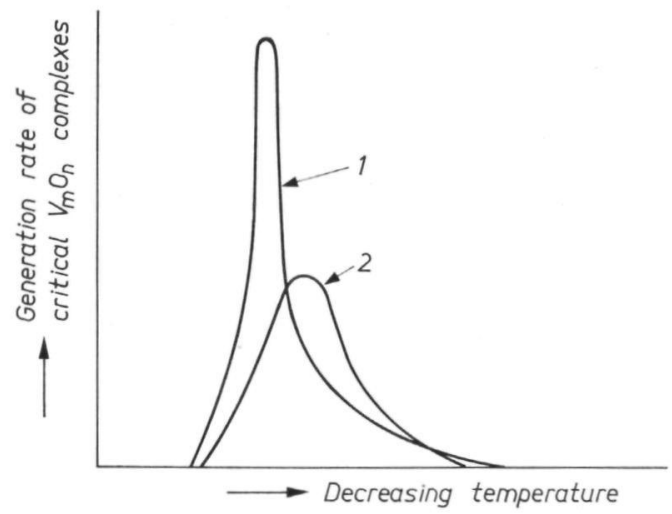

Fig. 4.3. The generation rate of critical $V_{m} O_{n}$ complexes during cooling of crystals with different oxygen concentrations. $N_{\mathrm{ox}}$ (curve 1) $>N_{\mathrm{ox}}$ (curve 2). 
temperature interval $T_{c}-T_{f}$. The difference in complex concentration will therefore be less than $F^{n}$.

We will now discuss the influence of the crystal-growth rate $V_{g}$ on the formation of critical nuclei. When $V_{g}$ is increased the crystal-cooling rate is also increased, because the axial temperature gradient is found to be only slightly dependent on $V_{g}$. Consequently, less time is available for complex formation in the temperature interval $T_{c}-T_{f}$ and a decrease of $N_{\mathrm{A}}$ would be expected. This is not observed for the range of growth rates employed. Crystals grown in argon at $1 \mathrm{~mm} / \mathrm{min}$ and $3 \mathrm{~mm} / \mathrm{min}$ were found to contain about the same A-cluster concentrations (figs 3.10 and 3.11 ). This is probably due to the following process. As in the case of homogeneous nucleation, the formation of $\mathrm{V}_{m} \mathrm{O}_{n}$ complexes, acting as critical nuclei, will exhibit a certain incubation time $t_{0}$ required for the consecutive association of vacancies and oxygen atoms. Consequently, when the growth rate, and hence the cooling rate, is increased (fig. 4.4), the temperature $T_{c}$ present after the incubation time $t_{0}$ has elapsed

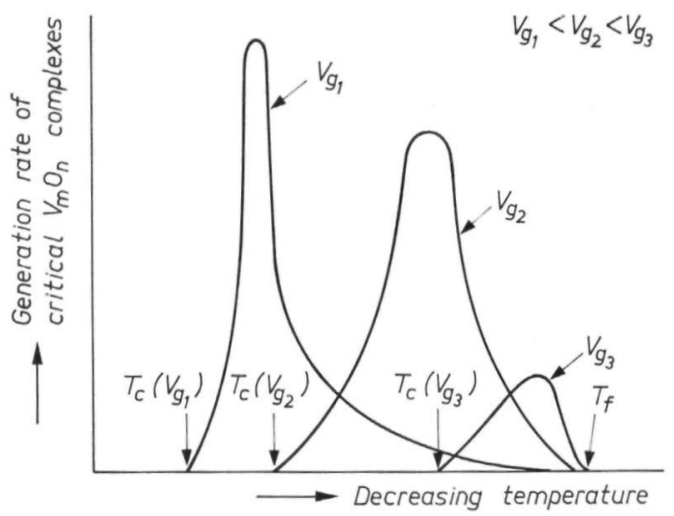

Fig. 4.4. The change of the generation rate of critical $V_{m} O_{n}$ complexes during cooling due to an increase in growth rate $\left(V_{g}\right)$. A marked reduction in the generation rate of complexes occurs as soon as $T_{c}$ approaches $T_{f}$.

shifts to a lower value. Moreover, the incubation time will increase with increasing cooling rate because of the faster reduction of $D_{\text {ox }}(T)$ and $D_{\mathrm{V}}(T)$. A pronounced reduction of the concentration of $\mathrm{V}_{m} \mathrm{O}_{n}$ complexes and consequently a strong reduction of $N_{\mathrm{A}}$ will only occur as soon as $T_{c}$ approaches $T_{f}$ $\left(V_{g}=V_{g_{3}}\right)$. This obviously requires growth rates larger than $3 \mathrm{~mm} / \mathrm{min}$.

In conclusion it can be said that growth rates up to $3 \mathrm{~mm} / \mathrm{min}$ have no influence on the value of $N_{\mathrm{A}}$, while variation of $N_{\text {ox }}$ has a smaller effect than expected. This may explain why differences in $N_{\mathrm{A}}$ larger than a factor of 20 have not been found between the various crystals investigated.

$\operatorname{Re}(b)$. It was assumed that the vacancy distribution present after solidification is uniform (sec. 4.5). This, however, does not apply to the oxygen distri- 
bution. Because the distribution coefficient of this impurity is $0 \cdot 5^{4-22}$ ), the oxygen concentration will show a periodic variation along the growth axis (oxygen striations, curve 1, fig. 4.5). The amplitude of the concentration modulation depends on the variation of growth rate during crystal rotation (eq. (1.1)).

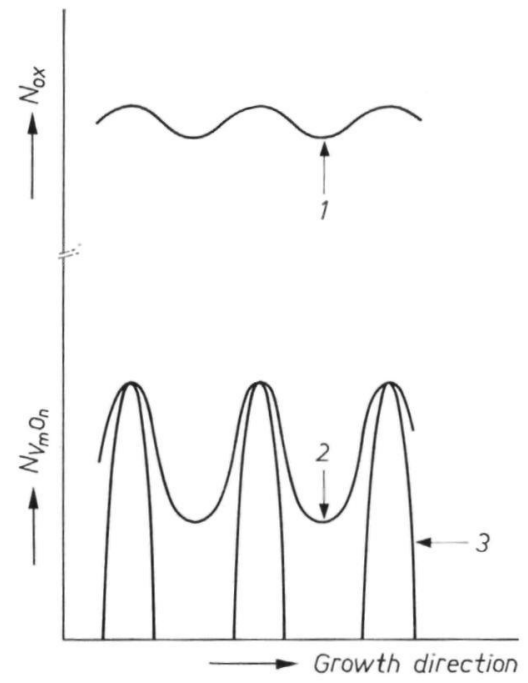

Fig. 4.5. Oxygen and $V_{m} O_{n}$-complex concentration versus position along the growth direction. Curve 1: periodic modulation of $N_{\mathrm{ox}}$. Curve 2: periodic variation in the concentration of $V_{m} O_{n}$ complexes $\left(\approx N_{o x}{ }^{n}\right)$. Curve 3: enhanced modulation of the concentration of $V_{m} O_{n}$ complexes due to the existence of an incubation time.

The generation rate of the $\mathrm{V}_{m} \mathrm{O}_{n}$ complexes is proportional to $\left[N_{\mathrm{V}}\right]^{m}\left[N_{\mathrm{ox}}\right]^{n}$. Since $n>1$ the $\mathrm{V}_{m} \mathrm{O}_{n}$ complexes and consequently the vacancy clusters are formed in a much more pronouncedly striated distribution than that of oxygen itself (fig. 4.5, curve 2). It is frequently observed that the minima in the A-cluster distribution are almost equal to zero (fig. 4.5, curve 3 ) as, for instance, in the crystals shown in figs 3.9 and 3.15 . This will be due to the following process. The incubation time $t_{0}$ depends on the value of $N_{\mathrm{ox}}$. In the regions of maximum $N_{\text {ox }}$ the incubation time will be shortest (fig. 4.6) and consequently the first complexes will be formed there. Calculation of the diffusion-limited annihilation of vacancies on these complexes shows that at high temperatures the supersaturation with vacancies decreases steeply within a few seconds. This reduces the generation rate for new complexes to very low values before any complexes are formed in the regions of minimum $N_{\mathrm{ox}}$. This explains the pronouncedly striated pattern for A-clusters. The same explanation does not hold for the B-clusters, because a fraction of the $\mathrm{V}_{m^{\prime}} \mathrm{O}_{n^{\prime}}$ complexes has already been formed at temperatures above $T_{c^{\prime}}$. Incubation-time effects on the distribution 


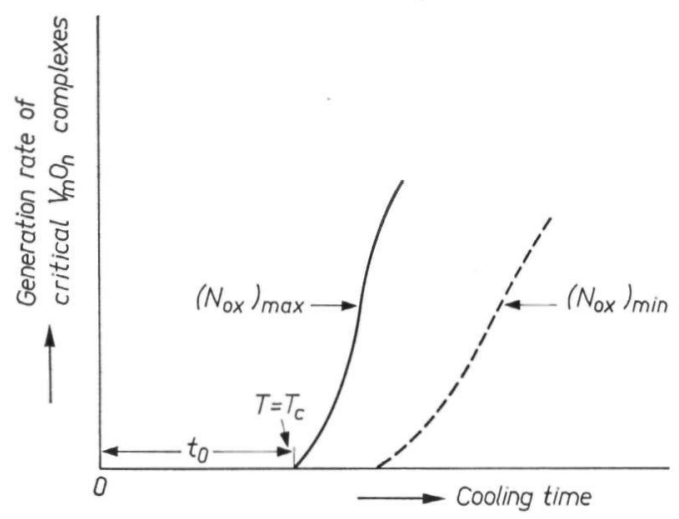

Fig. 4.6. The generation rate of critical $V_{m} O_{n}$ complexes versus cooling time. The incubation time for complex formation will be minimum $\left(t_{0}\right)$ in the regions containing the highest oxygen concentration.

of these complexes are therefore less marked. This explains why the modulation in the distribution of B-clusters is less pronounced (fig. 3.8).

The periodicity of the variation in growth rate induced by crystal rotation will be disturbed by random fluctuations in the thermal-convection pattern present in the molten zone. These fluctuations will introduce random variations in the melt temperature near the solid-liquid interface and hence in growth rate. This results in random disturbances in the oxygen striations, which explains the interruptions observed in the cluster distribution. The extremely disturbed cluster pattern observed in the crystal shown in fig. 3.16 is attributed to non-coinciding rotation axes of the crystal and the polycrystalline feed-rod.

$\operatorname{Re}(\mathrm{c})$. The crystal surface region will exhibit a less marked tendency for vacancy-oxygen-complex formation for a variety of reasons:

(1) The vicinity of the surface, acting as a vacancy sink, will keep the supersaturation low during cooling.

(2) The cooling rate near the surface is larger than in the bulk of the crystal. Consequently, less time will be available for complex formation.

(3) The oxygen concentration will be lower near the surface. Using infrared spectroscopy, Kaiser et al. ${ }^{4-23}$ ) detected in Czochralski-grown silicon crystals the presence of a surface layer, about $2 \mathrm{~mm}$ in width, containing much less oxygen than that found in the bulk. This layer was attributed to a depletion of oxygen at the melt surface due to $\mathrm{SiO}$ evaporation from the melt. The same effect, which will be maximum for crystal growth under high-vacuum conditions, can also be expected in floating-zone silicon.

The above-mentioned processes obviously cause complete elimination of the A-clusters in a surface layer of about $2 \mathrm{~mm}$ in width (figs $3.1,3.7$ and 3.14), while B-clusters are still formed in the argon-grown material. This indicates 
that the nucleation sites for B-clusters are more easily formed. The absence of the B-clusters near the surface of vacuum-grown crystals (figs 3.14 and 3.15) is presumably due to the more pronounced $\mathrm{SiO}$ evaporation from the melt. This supports the original assumption that oxygen plays an essential part in the formation of nuclei.

The explanation for the different cluster distribution near the surface also holds for the upper part of crystal cone region II (figs 3.7(a) and 3.12(b)).

The absence of vacancy clusters in the dislocation-free area of the thin neck (crystal region I) of argon-grown silicon will be due to the extremely fast cooling rate. This region (radius: $0.15 \mathrm{~cm}$ ) is grown at a rate of $15 \mathrm{~mm} / \mathrm{min}$, while the axial temperature gradient is about $1000^{\circ} \mathrm{C} / \mathrm{cm}^{4-24}$ ). Consequently, the cooling rate is about $25^{\circ} \mathrm{C} / \mathrm{s}$, whereas for the crystal region III this amounts to 0.5 to $1.5^{\circ} \mathrm{C} / \mathrm{s}$. Using eq. (4.1) we find that when the neck is cooled down to $400{ }^{\circ} \mathrm{C}, N_{\mathrm{v}}(r=0) / N_{\mathrm{v}}\left(T_{M}\right)>0.99$, indicating that out-diffusion of vacancies in region $\mathrm{I}$ is negligible. Consequently, the absence of cluster formation must be due to the prevention of diffusion-limited formation of nuclei during rapid cooling. Since diffusion of vacancies is still possible at room temperature, the absence of cluster formation during fast cooling indicates that the limiting process in the formation of nuclei is due to the participation of oxygen. This implies that the nuclei for both the A- and B-clusters contain more than one oxygen atom.

As discussed in sec. 3.3, a pronounced remelt $\left(V_{g}<0\right)$ occurs in crystal region IV, where the growth process is terminated. This introduces large variations in growth rate around the average growth rate $V_{g}$, and therefore in $N_{\text {ox }}$, which accounts for the increase in $N_{\mathrm{A}}$ found in this region (fig. 3.7(c)).

$\operatorname{Re}(\mathrm{d})$. The typical minimum in the A-cluster distribution observed in the central region of most of the crystals (e.g. fig. 3.15), is attributable to two cooperative processes. Firstly, the periodic variation in growth rate and consequently in $N_{o x}$ will generally increase in passing from the crystal rotation axis towards the periphery of the crystal ${ }^{4-25}$ ). The generation of $\mathrm{V}_{m} \mathrm{O}_{n}$ complexes, which mainly occurs in the layers where $N_{\mathrm{ox}}=\left(N_{\mathrm{ox}}\right)_{\max }$, will therefore increase with distance from the rotation axis. Secondly, owing to the radial temperature gradient present during cooling of the crystal, the first $\mathrm{V}_{m} \mathrm{O}_{n}$ complexes will be formed in the cylindrical region $P$ (fig. 4.7) where the temperature drops below $T_{c}$. As soon as clusters are formed in this region the supersaturation of vacancies decreases rapidly. This causes a large radial gradient in $N_{\mathrm{v}}$ at the junction between region $\mathrm{P}\left(T<T_{c}\right)$ and $\mathrm{Q}\left(T>T_{c}\right)$. This will enhance the diffusion of vacancies from region $Q$ into region $P$. These vacancies will contribute to the formation of extra $\mathrm{V}_{m} \mathrm{O}_{n}$ complexes. Further cooling will cause the $\mathrm{P}-\mathrm{Q}$ junction to move inwards until the crystal centre is reached. Complex formation will then start at the centre as well. The supersaturation in 


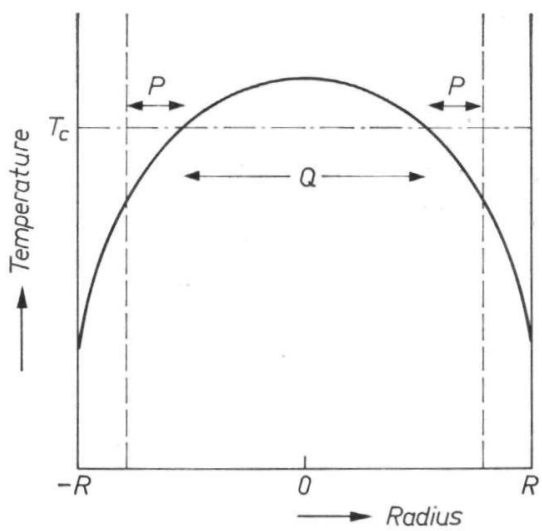

Fig. 4.7. Radial temperature distribution in the crystal area where the formation of critical $V_{m} O_{n}$ complexes begins (region $\mathrm{P}$ ).

$N_{\mathrm{v}}$ in the centre, however, will already be greatly reduced by the vacancy diffusion towards the original $\mathrm{P}$ regions.

Summarizing it can be said that all the experimental observations can be understood on the basis of the discussed model for heterogeneous nucleation of vacancies. This model describes the formation of $\mathrm{V}_{m} \mathrm{O}_{n}$ complexes containing more than one oxygen atom, which act as critical nuclei for vacancy condensation. Moreover, this model also enables us to develop several crystal-growth methods with which a reduction or even an elimination of vacancy-cluster formation during growth can be obtained. In the next chapter these methods will be described together with the experimental results obtained by applying them.

\section{REFERENCES}

4-1) J. Crank, The mathematics of diffusion, Clarendon Press, Oxford, 1970.

4-2) A. G. Tweet, J. appl. Phys. 29, 1520, 1958.

4-3) A. G. Tweet, J. appl. Phys. 30, 2002, 1959.

4-4) A. J. R. de Kock, F. M. Beeftink and K. J. Sche11, Appl. Phys. Letters 20, 81, 1972.

${ }^{4-5}$ ) F. C. Frank, Madrid conference on deformation and flow of solids, Springer Verlag, Berlin, 1956, p. 73.

4-6) P. B. Hirsch, J. Silcox, R. E. Smallman and K. H. Westmacott, Phil. Mag. 3, $897,1958$.

4-7) T. Evans and C. Phaal, Phil. Mag. 7, 843, 1962.

4-8) D. Kuhlmann-Wilsdorf and H. G. F. Wils dorf, J. appl. Phys. 31, 516, 1960.

4-9) J. Hornstra, private communication.

4-10) G. Möllenstedt, Optik 10, 72, 1953.

4-11) A. Seeger and M. L. Swanson, in R. R. Hasiguti (ed.), Lattice defects in semiconductors, Univ. of Tokyo Press, 1968, p. 93.

4-12) J. W. Ferman and R. A. Swalin, in De Boer (Ed.), Reactivity of solids, (Symp. Amsterdam 1962), Elsevier Publ. Comp., 1962, p. 264.

4-13) F. S. Ham, J. Phys. Chem. Solids 6, 335, 1958.

4-14) E. M. Pell, Symposium on solid state physics and telecommunications (Brussels 1958), Academic Press, New York, 1960, vol. 1, p. 261. 
4-15) F. Morin and H. Reiss, J. Phys. Chem. Solids 3, 196, 1957.

4-16) T. S. Plaskett, Trans. AIME 233, 809, 1965.

4-17) W. W. Webb, J. appl. Phys. 33, 1961, 1962.

4-18) J. W. Corbett, G. D. Watkins and R. S. McDonald, Phys. Rev. 135, Al381, 1964.

4-19) R. J. Jaccodine, J. electrochem. Soc. 110, 524, 1963.

4-20) G. D. Watk ins, J. phys. Soc. Jap. 18, 22, 1963, Suppl. II.

4-21) R. A. Logan and A. S. Peters, J. appl. Phys. 30, 1627, 1959.

4-22) F. A. Trumbore, Bell Sys. tech. J. 39, 205, 1960.

4-23) W. Kaiser and P. H. Keck, J. appl. Phys. 28, 882, 1957.

4-24) G. Ziegler, Z. Naturforsch. 16a, 219, 1961.

4-25) K. Morizane, A. Witt and H. C. Gatos, J. electrochem. Soc. 114, 738, 1967. 


\section{THE ELIMINATION OF VACANCY-CLUSTER FORMATION DURING CRYSTAL GROWTH}

\subsection{Introduction}

During silicon-crystal growth the material becomes supersaturated with vacancies because the vacancy concentration decreases with decreasing temperature (eq. (1.2)). As discussed in sec. 4.6 this results in the formation of vacancy clusters in dislocation-free crystals via a heterogeneous nucleation process. Because a supersaturation with vacancies cannot be avoided, the cluster formation can only be reduced when the formation of nuclei (vacancy-oxygen complexes) is reduced. In this chapter three methods of bringing about a decrease in cluster concentration are described. The results obtained with these methods are discussed.

\subsection{Methods for the elimination of vacancy clusters}

\subsubsection{Method I}

The nuclei for cluster formation are assumed to be formed via a diffusionlimited association of vacancies and oxygen atoms. When the cooling rate of the crystal is sufficiently increased, less time is available for vacancy-oxygen association, resulting in a lower concentration of critical nuclei (see also sec. 4.7). The cooling rate of a crystal can be.speeded up by increasing the growth rate $V_{g}$ and by increasing the temperature gradients in the crystal during growth. The temperature gradients in the growing crystal can be increased by using a gas atmosphere with a large heat conductivity $\left(\mathrm{He}, \mathrm{H}_{2}\right)$ and a high pressure or by introduction of a forced convection along the crystal.

As discussed already in sec. 4.7 an increase in $V_{g}$ from 1 to $3 \mathrm{~mm} / \mathrm{min}$, resulting in a change of the cooling rate from about 0.5 to $1.5{ }^{\circ} \mathrm{C} / \mathrm{s}$, does not significantly alter $N_{\mathrm{A}}$ due to the fact that most $\mathrm{V}_{m} \mathrm{O}_{n}$ complexes are formed in a short temperature interval $T_{c}-T_{f}$. However, a cooling rate of about $25^{\circ} \mathrm{C} / \mathrm{s}$, such as occurs in the thin crystal neck (region I), seems to be sufficient to reduce $N_{\mathrm{A}}+N_{\mathrm{B}}$ to zero (sec. 4.7). It is likely, therefore, that there exists a critical cooling rate, somewhere between 1.5 and $25^{\circ} \mathrm{C} / \mathrm{s}$ which results in a complete suppression of nucleus formation. Crystals cooled at rates above this critical value will remain free from vacancy clusters. However, the growth of dislocation-free material under such conditions will be difficult for the following reasons.

(1) During fast crystal growth a large amount of latent heat of solidification is developed. This tends to increase the temperature near the solid-liquid interface, resulting in a decrease of the crystal diameter. This can only be prevented by the introduction of large temperature gradients in the growing 
crystal which enables a sufficiently fast transport of the latent heat away from the interface.

(2) At the required high growth rates spurious nucleation at the interface may prevent the growth of perfect single crystals.

(3) During fast cooling the formation of high radial temperature gradients in the crystal should be avoided because otherwise dislocations are introduced ${ }^{5-1}$ ).

An alternative cooling procedure could be as follows. During growth the whole crystal is kept at a temperature $T$, with $T_{c}<T<1420^{\circ} \mathrm{C}$ by means of a system of after-heaters. This prevents complex formation during growth whereas simultaneously excess vacancies can diffuse out towards the crystal surface. When the growth process is terminated the crystal is cooled down to room temperature at a rate which is sufficiently fast to prevent complex formation.

\subsubsection{Method II}

The generation rate of $\mathrm{V}_{m} \mathrm{O}_{n}$ complexes is proportional to $\left[N_{\mathrm{v}}\right]^{m}\left[N_{\mathrm{ox}}\right]^{n}$. Consequently, a reduction of the generation of nuclei can be obtained by lowering $N_{\text {ox }}$. However, as discussed in sec. 4.7, a decrease in $N_{\text {ox }}$ will cause a much smaller reduction in $N_{\mathbf{v}_{m} \mathrm{O}_{n}}$ than expected (see fig. 4.3). Because floatingzone silicon usually contains $10^{14}$ to $10^{16}$ oxygen atoms per $\mathrm{cm}^{3}$ (sec. 1.3.1) a significant reduction in $N_{\mathrm{A}}$ can only be expected if $N_{\text {ox }} \ll 10^{14} \mathrm{at} / \mathrm{cm}^{3}$. This requires very clean growth conditions (e.g. ultra-high vacuum) and the use of extremely pure starting material. A difficulty in this respect may be the circumstance that, due to their strong reactivity, silicon surfaces are rapidly covered with at least one monolayer of oxide even under high-vacuum conditions ${ }^{5-2}$ ). This also applies to the surface of the polycrystalline rod-shaped starting material. If only $10 \%$ of the oxide monolayer is incorporated via the melt into the growing crystal this will result in an $N_{\mathrm{ox}}$ of about $10^{14} \mathrm{~cm}^{-3}$ when the crystal diameter amounts to $2.5 \mathrm{~cm}$.

Apart from by the lowering of $N_{\mathrm{ox}}$, the generation of nucleation sites will also be influenced by a reduction of the periodic variation in growth rate. The resulting lower values for $\left(N_{\mathrm{ox}}\right)_{\max }$ in the oxygen striations will certainly cause a more uniform distribution of vacancy-oxygen complexes. However, the reduction of the concentration of clusters, especially the B-clusters, will probably be small.

\subsubsection{Method III}

The third method of cluster reduction is based on the following trend of thought. Suppose one adds to the growing crystal an impurity $X$ which reacts with the oxygen atoms present in the silicon lattice. The $\mathrm{O}-\mathrm{X}$ bonds formed will lower the effective diffusion coefficient of oxygen. Either the $\mathrm{O}-\mathrm{X}$ associates have to dissociate in order to make interstitial diffusion of oxygen atoms pos- 
sible or the $\mathrm{O}-\mathrm{X}$ associates diffuse as a whole. In the first case a higher activation energy for migration than for single oxygen atoms can be expected while this probably also applies to the second case. The diffusion-limited formation of nucleation sites will, therefore, proceed more slowly. Furthermore, because of the $\mathrm{O}-\mathrm{X}$ interaction, the tendency for vacancy-oxygen association will be lowered as well.

In order to be useful the impurity $\mathrm{X}$ should meet the following four requirements.

(1) It should be possible to add the impurity $\mathrm{X}$ during crystal growth in sufficiently large quantities.

(2) The impurity $X$ should have an affinity to oxygen in the silicon lattice which is at least comparable with the affinity of vacancies to oxygen.

(3) Because the $\mathrm{O}-\mathrm{X}$ reactions should take place before the $\mathrm{V}_{m} \mathrm{O}_{n}$ association starts, $\mathrm{X}$ should possess a high diffusion coefficient in silicon compared with oxygen.

(4) In order to influence the electrical properties of the crystal as little as possible the impurity $X$ should not have donor or acceptor character.

The only impurity that meets all these requirements is hydrogen. Hydrogen can readily be added to the material by growing the crystal in a hydrogencontaining atmosphere. Depending on the partial pressure of $\mathrm{H}_{2}$ a certain amount of this impurity dissolves into the molten zone and subsequently is partly incorporated into the growing crystal.

Infrared-absorption measurements of dissolved oxygen at $9 \mu \mathrm{m}$ wavelength, reported by Yukimoto ${ }^{5-3,4}$, proved the existence of a hydrogen-oxygen interaction. Crystals $\left(10^{17} \mathrm{~cm}^{-3}<N_{\mathrm{ox}}<10^{18} \mathrm{~cm}^{-3}\right)$, grown from a silica crucible in $\mathrm{Ar}$ and $\mathrm{Ar}-\mathrm{H}_{2}$ mixtures, were compared. The $\mathrm{H}$-doped crystals exhibited a significantly lower absorption at $9 \mu \mathrm{m}$ than crystals grown in argon, indicating that a smaller amount of free interstitial oxygen atoms was present in the former. Annealing of the H-doped crystals at $1000{ }^{\circ} \mathrm{C}$ resulted in an increase of the $9-\mu \mathrm{m}$ absorption peak, pointing to an increase in the concentration of free oxygen atoms. It was assumed ${ }^{5-3,4}$ ) that during crystal growth $\mathrm{H}$-clusters are formed around oxygen atoms. During annealing at $1000^{\circ} \mathrm{C} \mathrm{H}$ diffuses out and the $\mathrm{H}$-clusters dissolve again. This explains the increase in the concentration of free oxygen atoms.

Hydrogen atoms are the fastest-diffusing impurities in silicon. Van Wieringen et al. $\left.{ }^{5-5}\right)$ found for the diffusion coefficient: $D_{\mathrm{H}}(T)=9 \cdot 4.10^{-3} \exp (0.48 \mathrm{eV} / k T)$. For instance $D_{\mathrm{H}}\left(1092^{\circ} \mathrm{C}\right)=1 \cdot 70 \cdot 10^{-4} \mathrm{~cm}^{2} / \mathrm{s}$, which is higher than $D_{\text {ox }}$ by more than six orders of magnitude. At these elevated temperatures hydrogen is even much more mobile than monovacancies (eq. (1.4)).

Finally, hydrogen does not exhibit donor character as measured by Crawford et al. ${ }^{5-6}$ ). This is in good agreement with a theoretical calculation by Reiss ${ }^{5-7}$ ), which showed that atomic hydrogen should fit entirely within the interstitial 
lattice positions. Hence its ionization potential is not appreciably affected by the dielectric constant of the silicon lattice.

It is now assumed that $\mathrm{H}$-doping of the growing crystal will reduce the association rate of vacancies and oxygen atoms. If this effect is combined with a sufficiently high cooling rate of the crystal a strong reduction or even a complete elimination of vacancy-oxygen-complex formation can be expected. As discussed in secs 4.7 and 5.2 .1 a critical cooling rate somewhere between 1.5 and $25{ }^{\circ} \mathrm{C} / \mathrm{s}$ is required for complex elimination without $\mathrm{H}$-doping. In the case of hydrogen addition this critical cooling rate should be lower.

\subsection{Experimental results and discussion}

\subsubsection{Methods I and II}

A few experiments have been carried out in order to investigate whether the basic principles of the methods I and II (secs 5.2.1 and 5.2.2) were correct.

A number of crystals were grown at 1 and $2 \mathrm{~mm} / \mathrm{min}$ in purified argon of atmospheric pressure. Via four nozzles a flow of purified argon (7-25.10 $\mathrm{cm}^{3} / \mathrm{min}$ ) was blown against the growing crystal. This introduced an increase in the cooling rate of the crystal surface region. This process caused a partial elimination of the B-clusters, which are otherwise present in the surface region of argon-grown crystals.

An attempt was made to lower $N_{\text {ox }}$ (method II) by a purification treatment directly prior to the growth process itself. A molten zone was slowly moved down the polycrystalline pedestal $\left(N_{\mathrm{ox}}<10^{16} \mathrm{~cm}^{-3}\right)$ in high vacuum $\left(10^{-6} \mathrm{~mm}\right.$ $\mathrm{Hg}$ ). Mainly as a result of $\mathrm{SiO}$ evaporation from the molten zone ${ }^{5-8}$ ), a certain reduction of $N_{\text {ox }}$ is expected to take place. After this treatment the crystalgrowth apparatus was filled with purified argon and a single crystal was grown from the purified starting material. The cluster concentration $N_{\mathrm{A}}$, determined by copper decoration, amounted to $7.10^{5} \mathrm{~cm}^{-3}$. This is the same concentration as found for the vacuum-grown crystal shown in fig. 3.14 and it implies a reduction by a factor of 2 to 3 compared with argon-grown crystals without purification treatment.

The above-mentioned results indicate that methods I and II, although still requiring further study for obtaining satisfactory results, are basically correct.

\subsubsection{Method III}

In order to investigate whether $\mathrm{H}$-doping influences the formation of vacancy clusters in the way described in sec. 5.2.3, a series of crystals was grown by the pedestal-pulling method. The gas atmosphere consisted of purified argon with an addition of $10 \%$ hydrogen ${ }^{*}$ ). The total pressure amounted to $1 \mathrm{~atm}$. High-

*) This relatively low concentration of hydrogen has been chosen because it has been reported that high concentrations of hydrogen may introduce additional crystal defects ${ }^{5-3,4,9,10}$ ). 
resistivity starting material was used with $N_{\text {ox }}<10^{16} \mathrm{~cm}^{-3}$. The crystal diameters were between 23 and $25 \mathrm{~mm}$. Different cooling rates were obtained by choosing growth rates between 0.6 and $3 \mathrm{~mm} / \mathrm{min}$. The perfection of these crystals was studied by means of copper and lithium decoration combined with X-ray topography as well as by preferential etching.

\section{(a) Copper decoration}

Figure 5.1 shows an X-ray topograph of a copper-decorated cross-sectional slice taken from a crystal grown at a rate of $0.6 \mathrm{~mm} / \mathrm{min}$. The average concentration of A-clusters was measured to be $5.10^{6} \mathrm{~cm}^{-3}$. At this low growth rate hydrogen doping seems to have no influence on the cluster formation.

Figure 5.2 shows a similar topograph of a slice taken from a crystal grown at $1 \mathrm{~mm} / \mathrm{min}$. This moderate increase in growth rate already caused a strong reduction in $N_{\mathrm{A}}$ down to $8.10^{5} \mathrm{~cm}^{-3}$. Obviously the cooling rate of the crystal surface region is already sufficiently high to suppress the formation of both A- and B-clusters completely in this region.

The result obtained with a growth rate of $2 \mathrm{~mm} / \mathrm{min}$ is illustrated by the topograph shown in fig. 5.3. In the bulk of the crystal $N_{\mathrm{A}}$ decreased still further

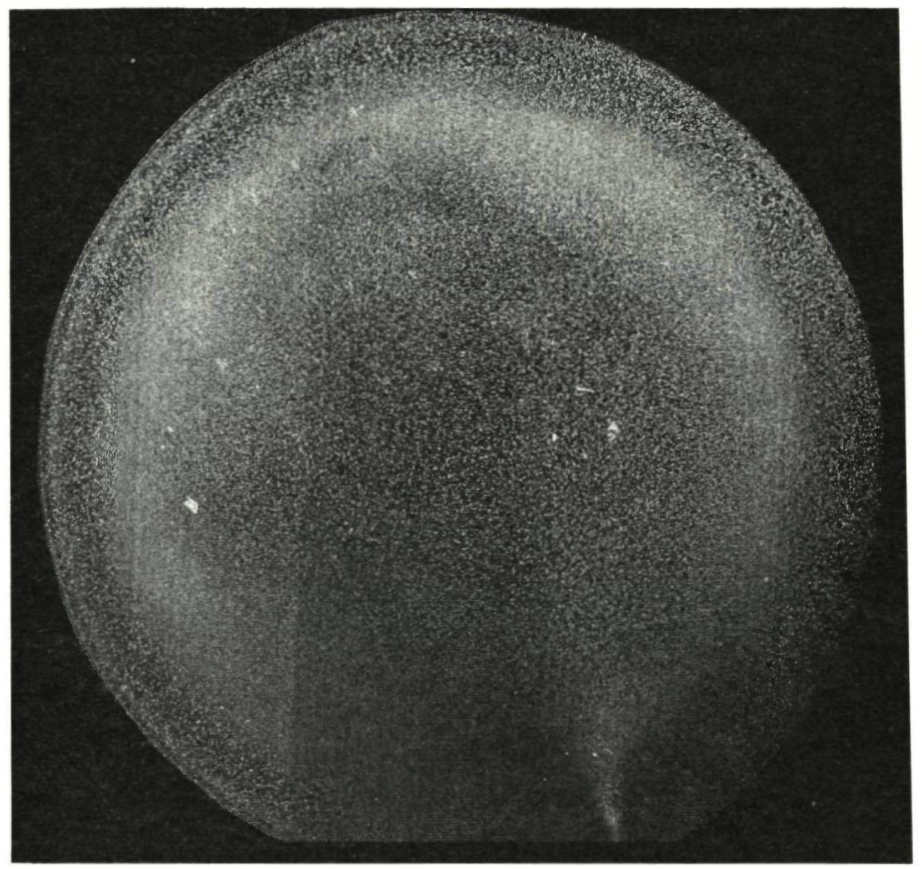

Fig. 5.1. X-ray transmission topograph of a copper-decorated cross-sectional slice taken from a pedestal-pulled crystal grown in $\mathrm{Ar}+10 \% \mathrm{H}_{2}$. Reflex: (220); $V_{g}=0.6 \mathrm{~mm} / \mathrm{min}$; $N_{\mathrm{A}}=5 \cdot 10^{6} \mathrm{~cm}^{-3}$. 


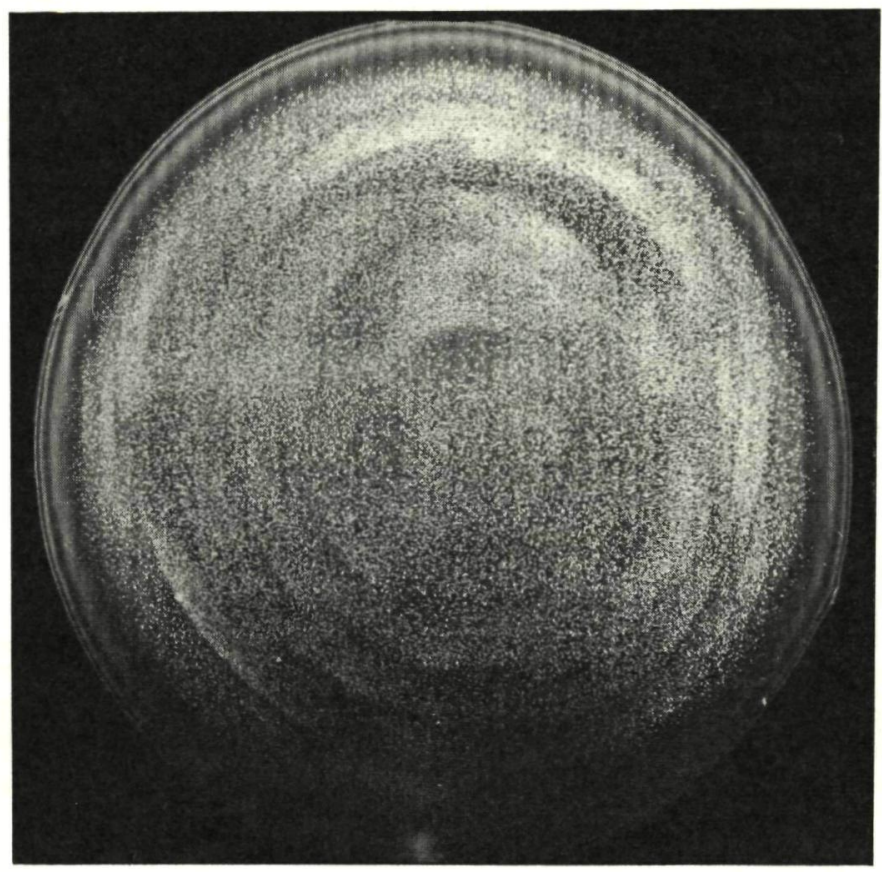

Fig. 5.2. X-ray transmission topograph of a copper-decorated cross-sectional slice taken from a pedestal-pulled crystal grown in $\mathrm{Ar}+10 \% \mathrm{H}_{2}$. Reflex: $(2 \overline{2} 0) ; V_{g}=1 \mathrm{~mm} / \mathrm{min}$; $N_{\mathrm{A}}=8 \cdot 10^{5} \mathrm{~cm}^{-3}$. 


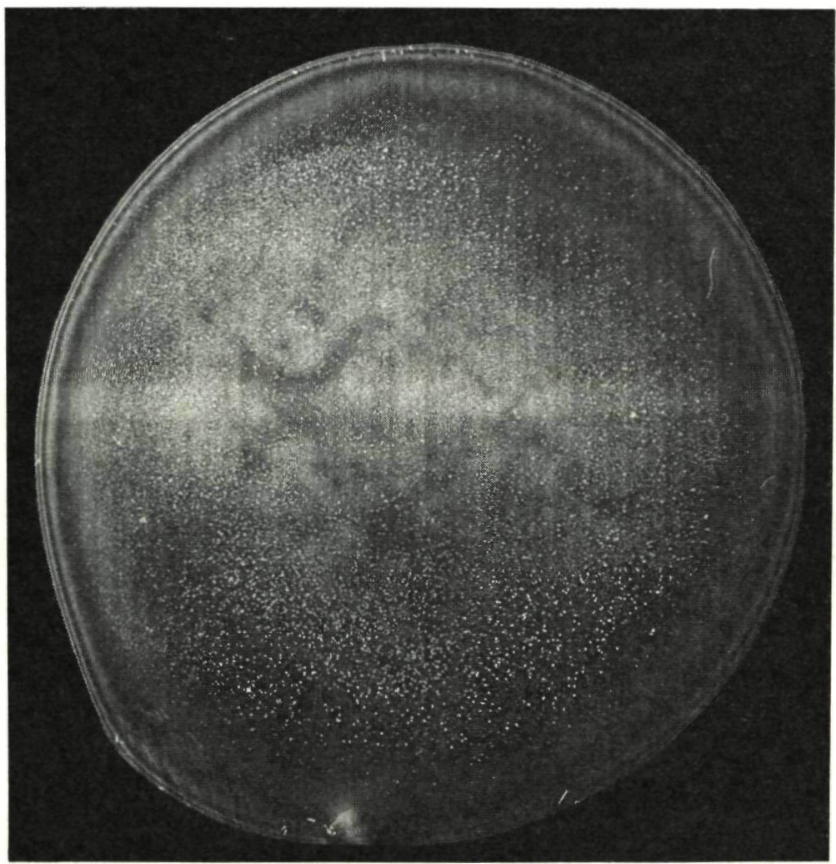

Fig. 5.3. X-ray transmission topograph of a copper-decorated cross-sectional slice taken from a pedestal-pulled crystal grown in $\mathrm{Ar}+10 \% \mathrm{H}_{2}$. Reflex: $(2 \overline{2} 0) ; V_{g}=2 \mathrm{~mm} / \mathrm{min}$; $N_{\mathrm{A}}=7 \cdot 10^{4} \mathrm{~cm}^{-3}, N_{\mathrm{B}}=3 \cdot 10^{6} \mathrm{~cm}^{-3}$. 
to $7.10^{4} \mathrm{~cm}^{-3}$. The cluster-free surface region became broader compared with material grown at $1 \mathrm{~mm} / \mathrm{min}$. Furthermore the B-clusters in this crystal are also decorated with copper. Consequently, their concentration could be determined directly from the topograph $\left(N_{\mathrm{B}}=3.10^{6} \mathrm{~cm}^{-3}\right)$. The cause of the decoration of the B-clusters will be threefold:

(1) because of the stronlgy reduced concentration of A-clusters more copper is available for decoration of the B-clusters;

(2) because of the low value of $N_{\mathrm{A}}$ the B-clusters capture more vacancies per defect; the resulting increase in size of the B-clusters will cause a stronger interaction with copper atoms;

(3) the concentration of B-clusters is reduced compared with normal material by about one order of magnitude (sec. 4.4).

When the growth rate was further increased to $3 \mathrm{~mm} / \mathrm{min}$ copper decoration showed that the formation of A-clusters has been completely eliminated (fig. 5.4). Various crystals, undoped as well as doped with phosphorus, have been grown at $3 \mathrm{~mm} / \mathrm{min}$. Complete elimination of the A-clusters was established in all these crystals. In the crystal shown in fig. $5.4, N_{\mathrm{B}}$ was reduced to zero

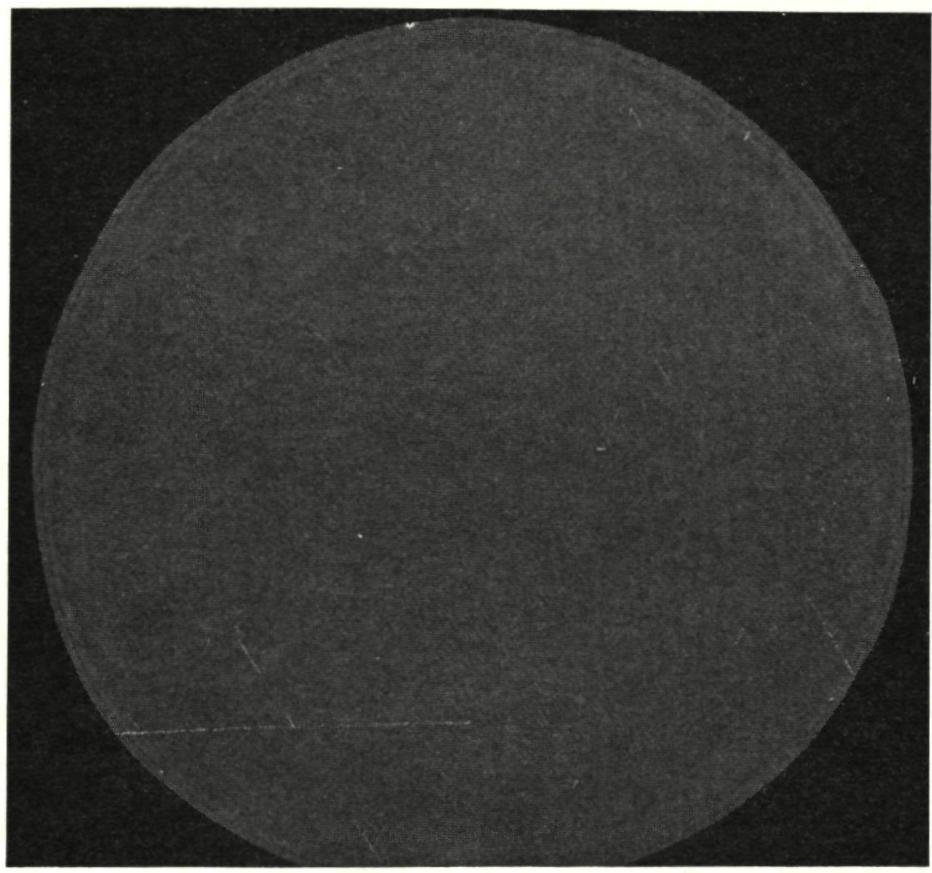

Fig. 5.4. X-ray transmission topograph of a copper-decorated cross-sectional slice taken from a pedestal-pulled crystal grown in $\mathrm{Ar}+10 \% \mathrm{H}_{2}$. Reflex: (220); $V_{g}=3 \mathrm{~mm} / \mathrm{min}$; $N_{\mathrm{A}}=0, N_{\mathrm{B}}=0$. A few dislocations are introduced during decoration. 
as well. However, a low residual concentration of the B-clusters in $\mathrm{H}$-doped crystals grown at $3 \mathrm{~mm} / \mathrm{min}$ was often detected.

(b) Etching

It was found that the presence of hydrogen in the silicon lattice prevented the detection of vacancy clusters by means of preferential etching. In crystals grown at for instance $1 \mathrm{~mm} / \mathrm{min}$, and which therefore still contained A- and B-clusters, no etch-pit pattern, such as shown in figs 3.1 and 3.2 was revealed after applying the etch treatment described in sec. 2.2.

However, when slices taken from crystals grown in $\mathrm{Ar}+10 \% \mathrm{H}_{2}$, and containing either vacancy clusters (type A) or dislocations, were non-preferentially elched in $\mathrm{HF}-\mathrm{HNO}_{3}$ mixtures, large holes were formed in the central region of the crystals, indicating the presence of large defects in this area. These holes were not formed during preferential etching. This peculiar etching behaviour is not well understood. Similar hole formation in H-doped crystals containing dislocations have previously been reported by Yoshimatsu ${ }^{5-9}$ ), Sugita ${ }^{5-10}$ ) and Yukimoto ${ }^{5-3,4}$ ). Sugita ${ }^{5-10}$ ), however, found that in his growth system a partial pressure of $\mathrm{H}_{2}$ of at least $0.25 \mathrm{~atm}$ had to be present in order to introduce these anomalous etching effects.

The appearance of the holes was found to be strongly dependent on the cooling rate of the crystals. Increasing the growth rate or applying a forced gas convection along the growing crystal increased the concentration and size of the holes in the crystal centre. Sometimes the defects causing these holes were revealed by X-ray topography. Figure 5.5 shows details of two topographs made of longitudinal slices cut from a crystal grown at $1 \mathrm{~mm} / \mathrm{min}$ in $\mathrm{Ar}+10 \% \mathrm{H}_{2}$. During growth, the radial temperature gradient was increased by means of forced gas convection in a way similar to that described in sec. 5.3.1. The upper half of the crystal was dislocation-free and contained vacancy clusters, whereas in the lower part dislocations were introduced. The topographs show dot-shaped defect images in the crystal centre at the location of the vacancy clusters (fig. 5.5(a)) and along the dislocation lines (fig. 5.5(b)). The detected strain fields are up to $100 \mu \mathrm{m}$ in diameter in this case. These defects are attributed to $\mathrm{H}$ precipitation on the grown-in defects. Although $\mathbf{H}$ is a fast-diffusing impurity, only a small amount will be able to diffuse out to the crystal surface during cooling. Consequently, considerable supersaturation will take place. Obviously, this supersaturation is eliminated by heterogeneous precipitation of $\mathrm{H}$ on the grown-in defects. The largest $\mathrm{H}$ precipitates are found near the crystal axis. This is probably due to the low cooling rate in this region so that more time is available for $\mathrm{H}$ precipitation.

It was established that the concentration of holes in the dislocation-free crystals is much smaller than $N_{\mathrm{A}}$. This indicates that large $\mathrm{H}$ precipitates are formed on a few clusters only. However, all the clusters can be expected to capture some 


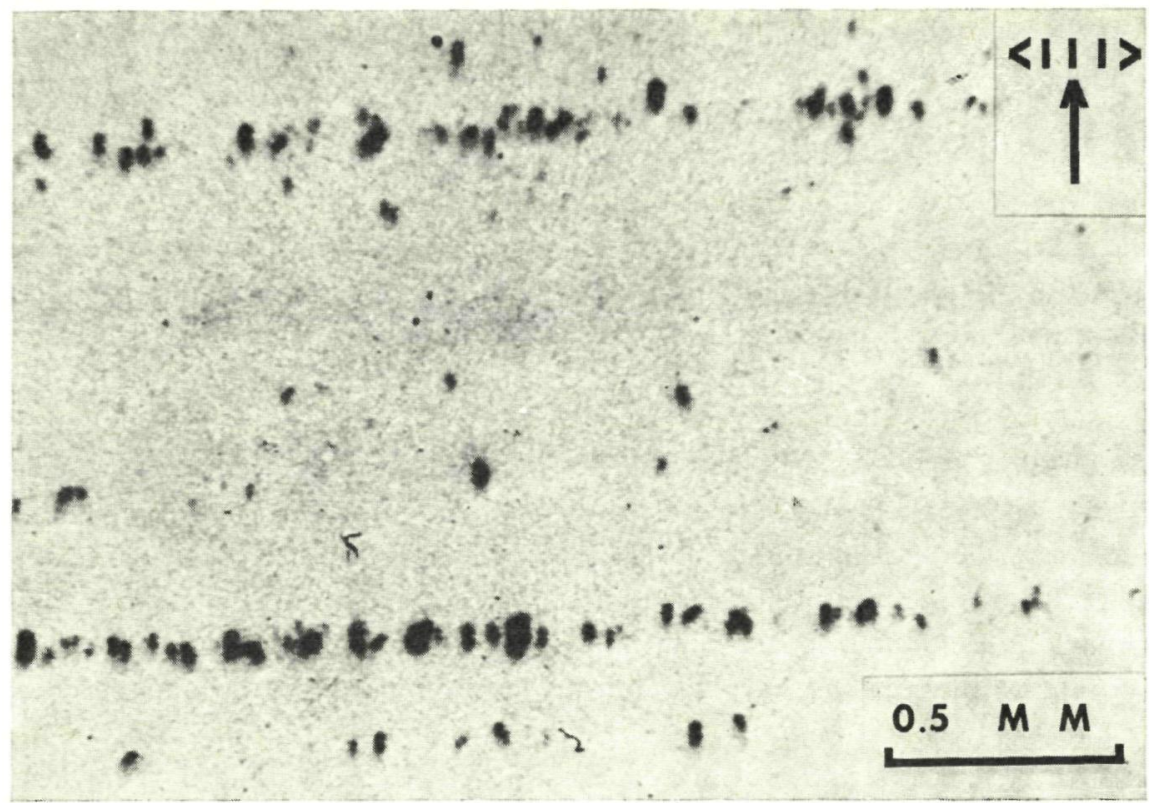

a)

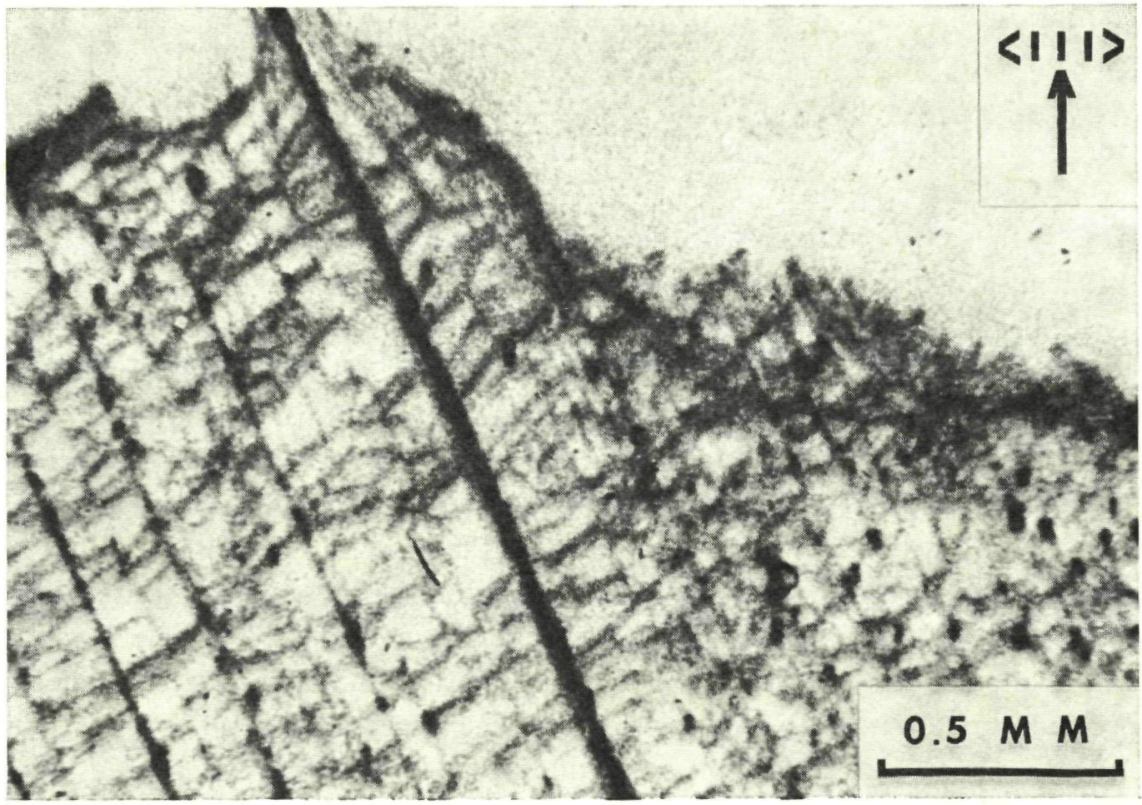

b)

Fig. 5.5. X-ray topographs of longitudinal sections taken from a crystal grown in $\mathrm{Ar}+10 \%$ $\mathrm{H}_{2}$. Reflex: (111); $V_{g}=1 \mathrm{~mm} / \mathrm{min}$. (a) Large strain fields around the vacancy clusters present near the axis of the crystal. (b) Large strain fields along the dislocations. 
hydrogen. It seems likely that this effect is the cause of the absence of vacancycluster etch-pit formation after preferential etching.

The $\mathrm{H}$ precipitates, formed on vacancy clusters as well as on dislocations, could be dissolved again by annealing treatments at $T \geqslant 550^{\circ} \mathrm{C}$. The defects, such as shown in fig. 5.5(a), were no longer revealed by $\mathrm{X}$-ray topography after the samples had been annealed for $3 \mathrm{~h}$ at $550^{\circ} \mathrm{C}$, whereas they disappeared after only a few minutes at $1000^{\circ} \mathrm{C}$. Simultaneously with the defect images in the topographs the anomalous etching effects disappeared. The low thermal activation required for dissolving the precipitates is consistent with the high diffusion coefficient of $\mathrm{H}$ in the silicon lattice.

When dislocation-free crystals are grown at $3 \mathrm{~mm} / \mathrm{min}$, resulting in the elimination of cluster formation, neither anomalous etching effects nor contrast in X-ray topographs is observed. Obviously, in the absence of grown-in defects, no large $\mathrm{H}$ precipitates are formed.

(c) Lithium decoration

Additional information about the crystal perfection of the H-doped dislocation-free crystals was obtained with the aid of lithium precipitation. Figure 5.6(a) shows the X-ray topograph of a longitudinal section of the same crystals as shown in fig. 5.2, which is grown at $1 \mathrm{~mm} / \mathrm{min}$ in $\mathrm{Ar}+10 \% \mathrm{H}_{2}$. Lithium was diffused in at $400{ }^{\circ} \mathrm{C}$ and the precipitation took place during annealing at $160{ }^{\circ} \mathrm{C}$. Apart from decoration of the A-clusters, a high density of lithium precipitates is detected in the crystal surface region where copper decoration shows that no vacancy clusters are present. A similar surface region, exhibiting strong lithium precipitation, has been found in germanium crystals grown in a hydrogen atmosphere ${ }^{5-11}$ ). The precipitates are formed in a slightly striated distribution. Figure $5.6(b)$ shows a sample of the same crystal after lithium diffusion at $500{ }^{\circ} \mathrm{C}$ and annealing at $160{ }^{\circ} \mathrm{C}$ for $41 \mathrm{~h}$. Again the A-clusters are decorated. Because more lithium is introduced at $500^{\circ} \mathrm{C}\left(C_{\mathrm{LI}}(0)=3.10^{18}\right.$ $\left.\mathrm{cm}^{-3}\right)$ than at $400{ }^{\circ} \mathrm{C}\left(C_{\mathrm{Li}}(0)=5.10^{17} \mathrm{~cm}^{-3}\right)$ the precipitates formed on the A-clusters are larger in size. However, the cluster-free surface region now exhibits hardly any precipitation. This indicates that a new type of nucleation site is present in such $\mathrm{H}$-doped crystals but dissolves at about $500^{\circ} \mathrm{C}$.

The electrically measured precipitation curves for the material grown at $1 \mathrm{~mm} / \mathrm{min}$ in $\mathrm{Ar}+10 \% \mathrm{H}_{2}$ are shown in fig. 5.7. The calculated concentration of lithium nucleation sites $\left(N_{\mathrm{p}}\right)$ for $T_{D}=400^{\circ} \mathrm{C}$ amounts to $2 \cdot 5 \cdot 10^{7}$ $\mathrm{cm}^{-3}$. For $T_{D}=500{ }^{\circ} \mathrm{C}$ we find $N_{\mathrm{p}}=4 \cdot 2 \cdot 10^{7} \mathrm{~cm}^{-3}$. Thus comparable values for $N_{\mathrm{p}}$ are found for these two different diffusion temperatures. Consequently, the new type of defect present near the surface is absent in the bulk of the crystal, so we can write: $N_{\mathrm{p}}\left(T_{\mathrm{D}}=400^{\circ} \mathrm{C}\right)=N_{\mathrm{A}}+N_{\mathrm{B}}$. Because $N_{\mathrm{A}}=8.10^{5} \mathrm{~cm}^{-3}$ (see fig. 5.2), $N_{\mathrm{B}} \approx 2 \cdot 5 \cdot 10^{7} \mathrm{~cm}^{-3}$.

The new type of defect is also found in vacancy-cluster-free material 


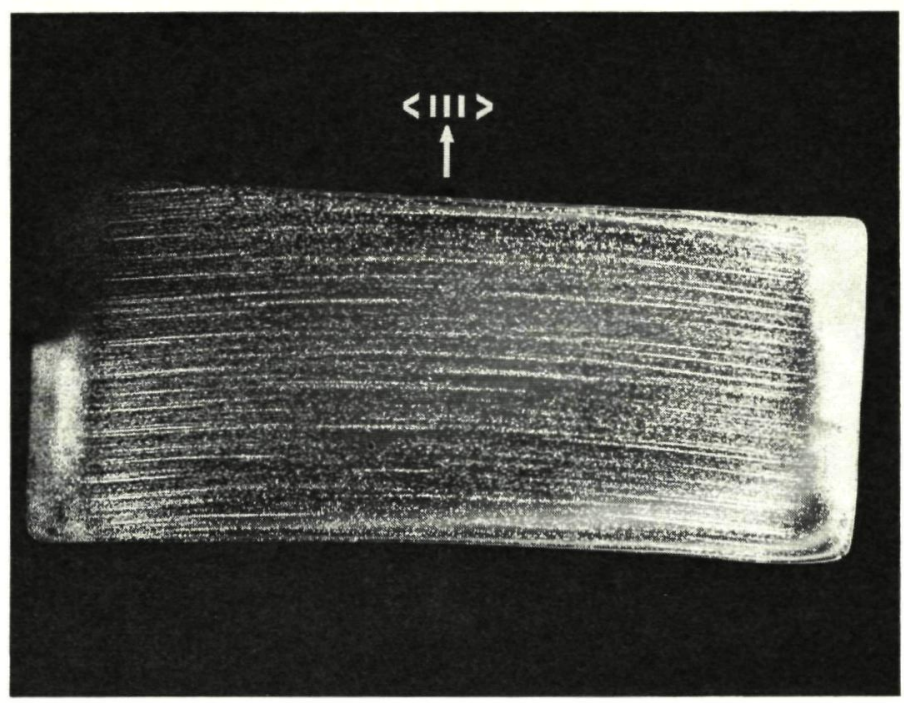

a)

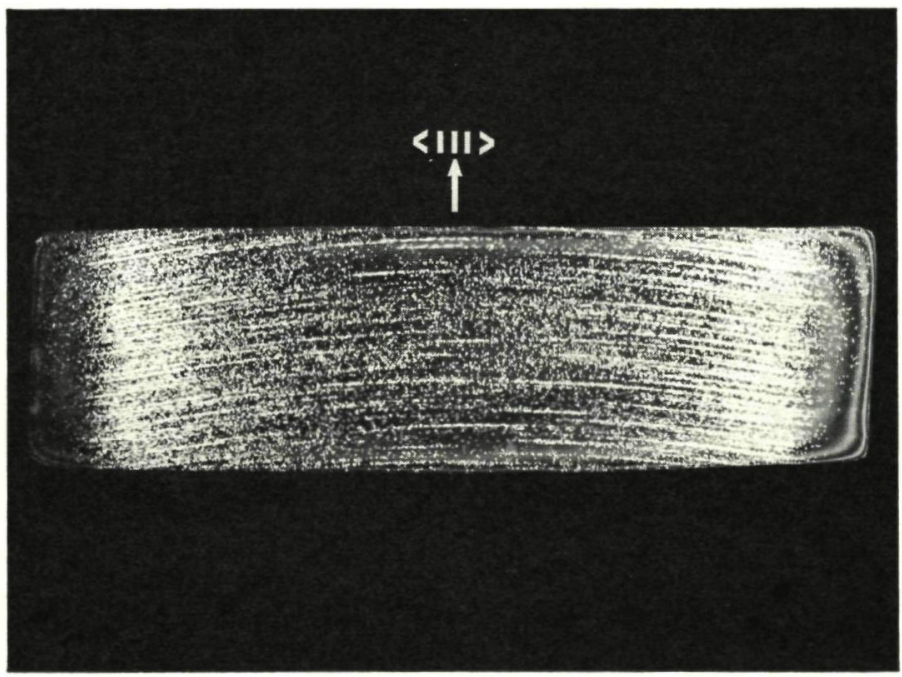

b)

Fig. 5.6. X-ray transmission topograph of a lithium-decorated longitudinal section of the same crystal as shown in fig. 5.2. Reflex: (111); $V_{g}=1 \mathrm{~mm} / \mathrm{min}$; atmosphere: $\mathrm{Ar}+10 \%$ $\mathrm{H}_{2}$. (a) $T_{D}=400{ }^{\circ} \mathrm{C}$; annealing time: $110 \mathrm{~h}$; surface region exhibits strong precipitation. (b) $T_{D}=500{ }^{\circ} \mathrm{C}$; annealing time: $41 \mathrm{~h}$; surface region exhibits hardly any precipitation. 


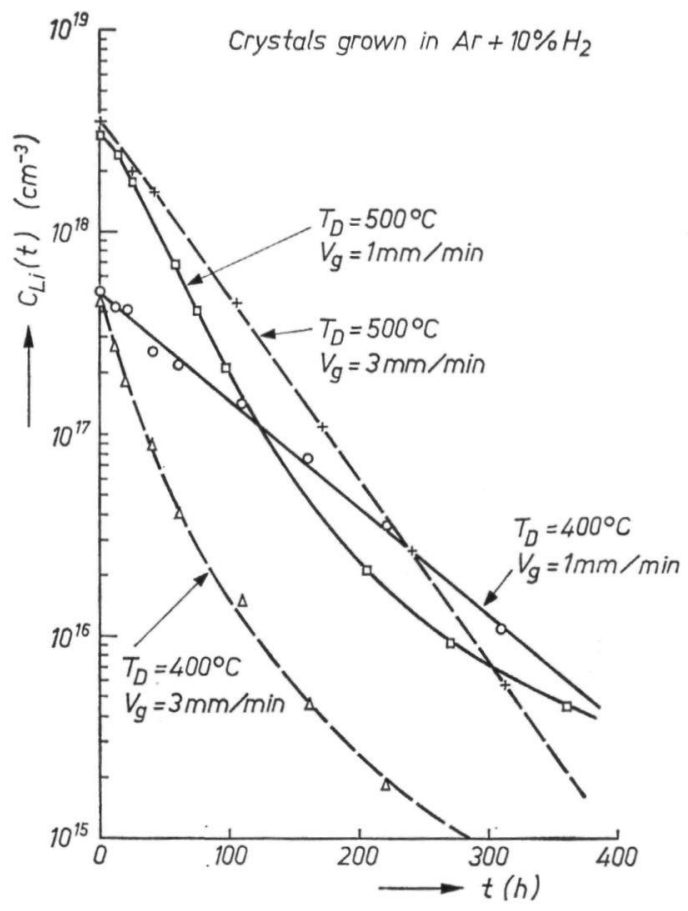

Fig. 5.7. Lithium-precipitation curves for the crystals shown in figs 5.2 and 5.4. $T_{D}=400$, $500{ }^{\circ} \mathrm{C}$; annealing temperature: $160{ }^{\circ} \mathrm{C}$; atmosphere: $\mathrm{Ar}+10 \% \mathrm{H}_{2}$. Drawn curves: crystal with $N_{\mathrm{A}}=8 \cdot 10^{5} \mathrm{~cm}^{-3}, V_{g}=1 \mathrm{~mm} / \mathrm{min}$. Dashed curves: cluster-free crystal $\left(N_{\mathrm{A}}=N_{\mathbf{B}}=0\right)$; $V_{g}=3 \mathrm{~mm} / \mathrm{min}$.

$\left(N_{\mathrm{A}}=N_{\mathrm{B}}=0\right.$, fig. 5.4), grown at $3 \mathrm{~mm} / \mathrm{min}$ in $\mathrm{Ar}+10 \% \mathrm{H}_{2}$. After diffusion at $400{ }^{\circ} \mathrm{C}$ into this material, X-ray topography showed lithium precipitation in a surface region of about $1 \mathrm{~mm}$ width, but hardly any contrast in the bulk of the crystal. The topographic result obtained with this crystal for $T_{D}=500{ }^{\circ} \mathrm{C}$ is shown in fig. 5.8. A low density $\left(2.10^{5} \mathrm{~cm}^{-3}\right)$ of large precipitates is detected in the bulk, which are located in a slightly striated configuration. The surface region contains a higher concentration of visible precipitates $\left(4.10^{6} \mathrm{~cm}^{-3}\right)$. The precipitation curves are plotted in fig. 5.7 $\left(T_{D}=400,500{ }^{\circ} \mathrm{C}\right)$. Calculation shows: $N_{\mathrm{p}}\left(T_{D}=400{ }^{\circ} \mathrm{C}\right)=1 \cdot 7 \cdot 10^{8} \mathrm{~cm}^{-3}$ and $N_{\mathrm{p}}\left(T_{D}=500{ }^{\circ} \mathrm{C}\right)=1 \cdot 9 \cdot 10^{7} \mathrm{~cm}^{-3}$. This indicates that in this crystal, as in the surface region of the crystal grown at $1 \mathrm{~mm} / \mathrm{min}$, the new type of nucleation sites is present and gradually dissolves at $500{ }^{\circ} \mathrm{C}$. The $N_{\mathrm{p}}$-values further indicate that only a fraction of the lithium precipitates is revealed by topography.

In table 5-I the calculated $N_{\mathrm{p}}$-values for the crystals, grown in $\mathrm{Ar}+10 \% \mathrm{H}_{2}$, are summarized. 


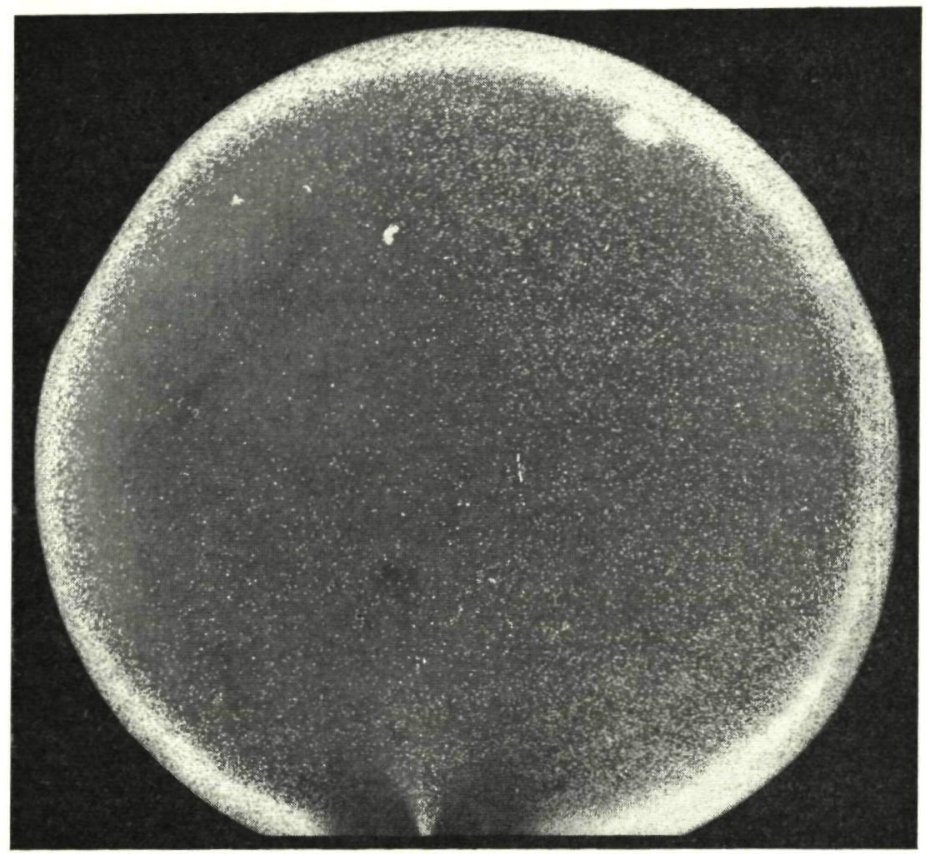

Fig. 5.8. X-ray transmission topograph of a lithium-decorated cross-sectional slice taken from the cluster-free crystal shown in fig. $5.4 . T_{D}=500{ }^{\circ} \mathrm{C}$; annealing time: $106 \mathrm{~h}$. Precipitate concentration in the central region: $2 \cdot 10^{5} \mathrm{~cm}^{-3}$ and near the surface: $4.10^{6} \mathrm{~cm}^{-3}$.

\section{TABLE 5-I}

The relation between the diffusion temperature of lithium and the calculated $N_{\mathrm{p}}$-values for crystals grown in $\mathrm{Ar}+10 \% \mathrm{H}_{2}$

\begin{tabular}{c|c|c}
\hline \multirow{2}{*}{$V_{g}(\mathrm{~mm} / \mathrm{min})$} & \multicolumn{2}{|c}{$N_{\mathrm{p}}\left(\mathrm{cm}^{-3}\right)$} \\
\cline { 2 - 3 } & $\begin{array}{c}T_{D}=400{ }^{\circ} \mathrm{C} \\
(\text { diffusion time: } \\
4 \cdot 5-5 \mathrm{~h})\end{array}$ & $\begin{array}{c}T_{D}=500{ }^{\circ} \mathrm{C} \\
\text { (diffusion time: } \\
6 \mathrm{~h})\end{array}$ \\
\hline 1 & $2 \cdot 5 \cdot 10^{7}$ & $4 \cdot 2 \cdot 10^{7}$ \\
3 & $1 \cdot 7 \cdot 10^{8}$ & $1 \cdot 9 \cdot 10^{7}$ \\
\hline
\end{tabular}


Preferential etching of carefully chemically-mechanically polished crosssectional slices, taken from the cluster-free crystal, causes the formation of tiny triangular shallow etch pits in a slightly striated distribution. The concentration of the defects causing these etch pits was estimated from the surface density of the pits in relation to the thickness of the layer removed by etching. It was established that the defect concentration in the bulk of the crystal amounted to about $10^{8} \mathrm{~cm}^{-3}$, whereas in a surface region, $1 \mathrm{~mm}$ in width, this concentration was $2.10^{9} \mathrm{~cm}^{-3}$. Because of the similarity of the distribution and concentration of the etch pits and the lithium precipitates formed for $T_{D}=400^{\circ} \mathrm{C}$, it is concluded that the two methods reveal the same defects. These defects, apparently gradually dissolving at $500{ }^{\circ} \mathrm{C}$, were found to be typical of perfect $\mathrm{H}$-doped crystals. It is concluded that these defects are tiny $\mathrm{H}$ precipitates formed in those areas where no other grown-in defects are present. In those perfect regions a high supersaturation with hydrogen builds up because no precipitation on vacancy clusters can take place. Finally, by some nucleation mechanism, hydrogen precipitates are formed. As mentioned in sec. 5.2.3, the $\mathrm{O}-\mathrm{H}$ interaction probably is partly responsible for the formation of $\mathrm{O}-\mathrm{H}$ clusters ${ }^{5-3,4}$ ). Because oxygen is present in a striated distribution (fig. 4.5), the striated distribution of the $\mathrm{H}$ precipitates can be understood. The presence of a thin surface layer containing a much higher concentration of $\mathrm{H}$ precipitates than in the bulk of the crystals will be due to the fast cooling rate at the surface. Although the surface, acting as a sink for hydrogen, is nearby, the fast cooling rate will cause a high supersaturation with hydrogen to occur in this area, resulting in the generation of a high concentration of nuclei. It should be emphasized that, if the nucleation of hydrogen occurs on oxygen atoms, the final distribution of $\mathrm{H}$ precipitates not only depends on the cooling procedure, but also on the distribution and concentration of the oxygen atoms.

Because of the competition between out-diffusion and precipitation the size and concentration of the $H$ precipitates near the surface will increase with increasing growth rate (cooling rate). This indeed is found. In contrast to the cluster-free material (growth rate $3 \mathrm{~mm} / \mathrm{min}$ ) the $\mathrm{H}$ precipitates, present near the surface in the crystal grown at $1 \mathrm{~mm} / \mathrm{min}$ (fig. 5.6), although detectable by lithium decoration, were too small to be revealed by preferential etching. This smaller size of the defects also explains why the defects in this crystal dissolve more rapidly at $500{ }^{\circ} \mathrm{C}$ than those in the cluster-free material.

\subsubsection{Differences in the formation of $\mathrm{V}-\mathrm{O}$ associates at $500{ }^{\circ} \mathrm{C}$ in crystals with} and without hydrogen dope

As described in sec. 3.4, lithium precipitation after diffusion at $T_{D} \leqslant 400{ }^{\circ} \mathrm{C}$ occurs solely on grown-in defects. No indications have been found that additional nucleation sites are formed at these diffusion temperatures. However, when lithium is diffused at $T_{D}=500{ }^{\circ} \mathrm{C}$ into dislocation-free silicon crystals, 
grown in argon or vacuum, a large number of newly formed nucleation sites is detected (sec. 3.5) which are not formed in $H$-doped crystals. These sites are distributed in a sharply defined striated pattern as shown in fig. 5.9. The precipitation curves $\left(T_{D}=400,500{ }^{\circ} \mathrm{C}\right)$ measured for argon- and vacuum-grown material are shown in fig. 5.10. It is calculated from these curves, using eqs (4.3) and (4.4), that after $6 \mathrm{~h}$ at $500{ }^{\circ} \mathrm{C}$ the concentration of the new centres formed in the argon-grown crystals amounted to $2 \cdot 5 \cdot 10^{9} \mathrm{~cm}^{-3}$ and to $10^{8} \mathrm{~cm}^{-3}$ in the vacuum-grown material (see table 5-II). Although lithium precipitation on the vacancy clusters still occurs, the presence of the new nucleation sites completely dominates the precipitation kinetics.

It is known, mainly from work by Ferman et al. ${ }^{5-12,13}$ ), that the $\mathrm{V}-\mathrm{O}$ pair acts as a preferred nucleation site for lithium. It is likely, however, that other types of vacancy-oxygen associates act as nucleation sites as well. After heating from room temperature to $500{ }^{\circ} \mathrm{C}$ the formation of stable $\mathrm{VO}_{3}$ complexes can be expected (sec. 1.3.3.3). These complexes will be formed in a sharply defined striated pattern (sec. 4.7). It is concluded, therefore, that the lithium nucleation sites formed at $500{ }^{\circ} \mathrm{C}$ are $\mathrm{VO}_{3}$ complexes. The high concentration in argongrown material compared with crystals grown in vacuum will be due to the lower value of $N_{\mathrm{ox}}$ in the latter. Furthermore, when $T_{D}=600{ }^{\circ} \mathrm{C}$ the new

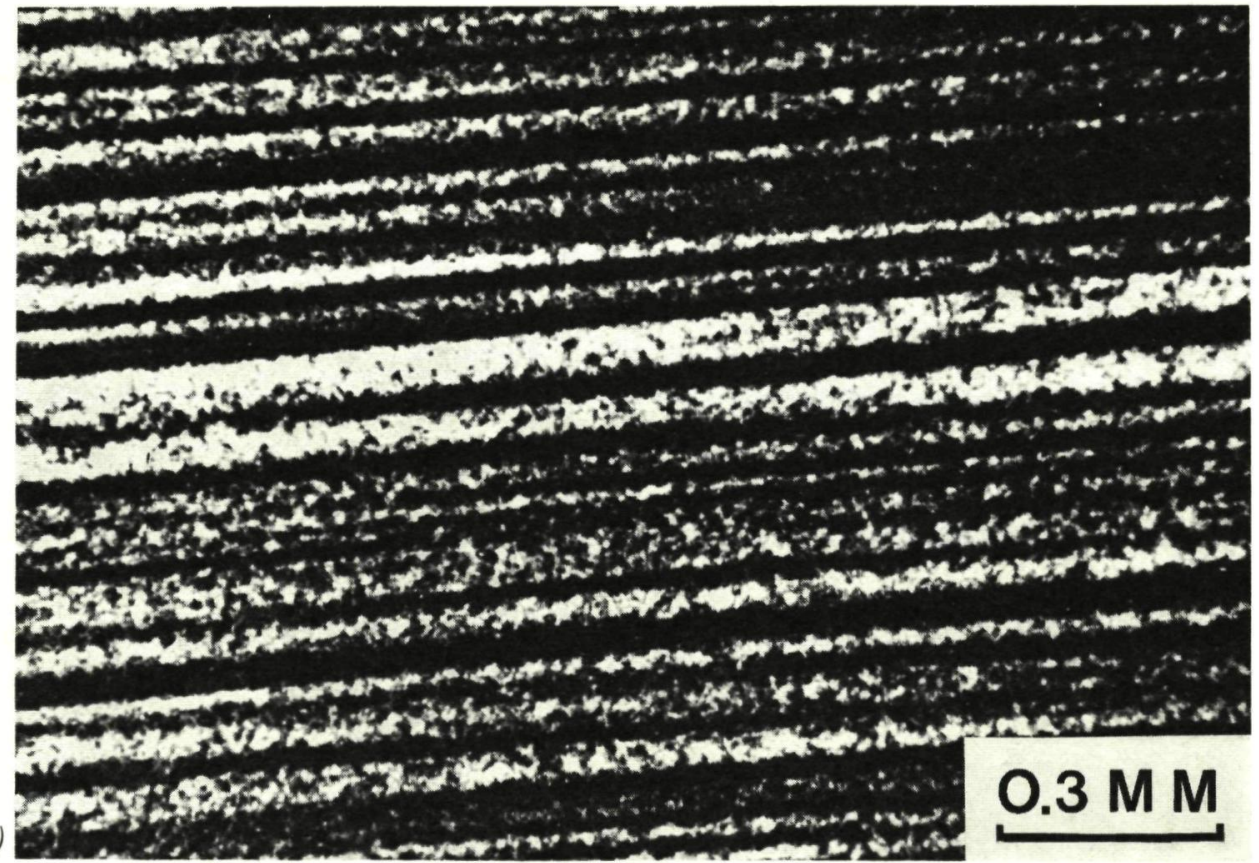

Fig. 5.9. X-ray topographs of longitudinal sections taken from dislocation-free crystals containing vacancy clusters. Lithium diffusion at $T_{D}=500^{\circ} \mathrm{C}$ during $6 \mathrm{~h}$. (a) Crystal grown in argon; annealing time after diffusion: $9 \mathrm{~h}$; concentration of newly generated nuclei : $2 \cdot 5 \cdot 10^{9}$ $\mathrm{cm}^{-3}$. 


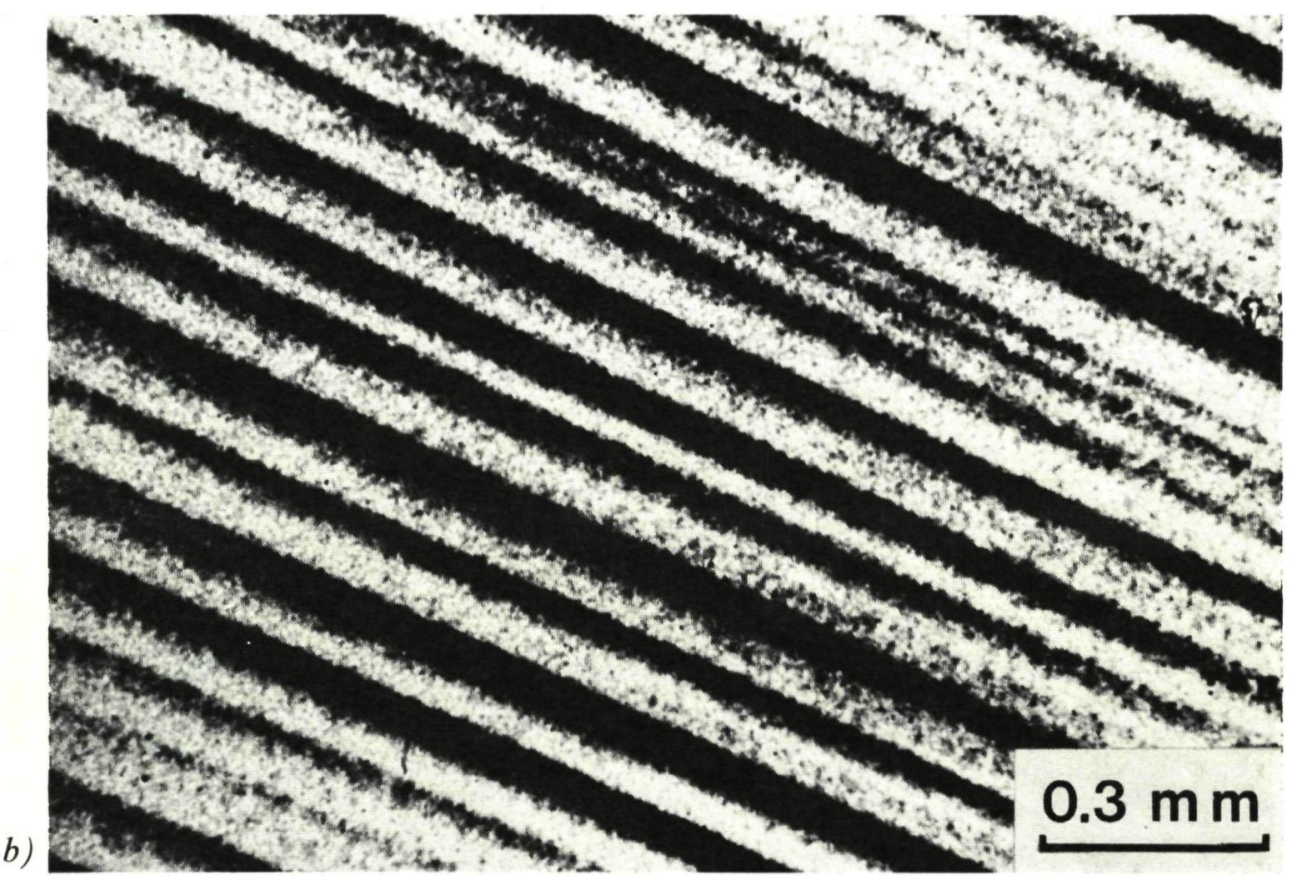

Fig. 5.9. (b) Crystal grown in vacuum; annealing time after diffusion: $1 \mathrm{~h}$; concentration of newly generated nuclei: $10^{8} \mathrm{~cm}^{-3}$. 


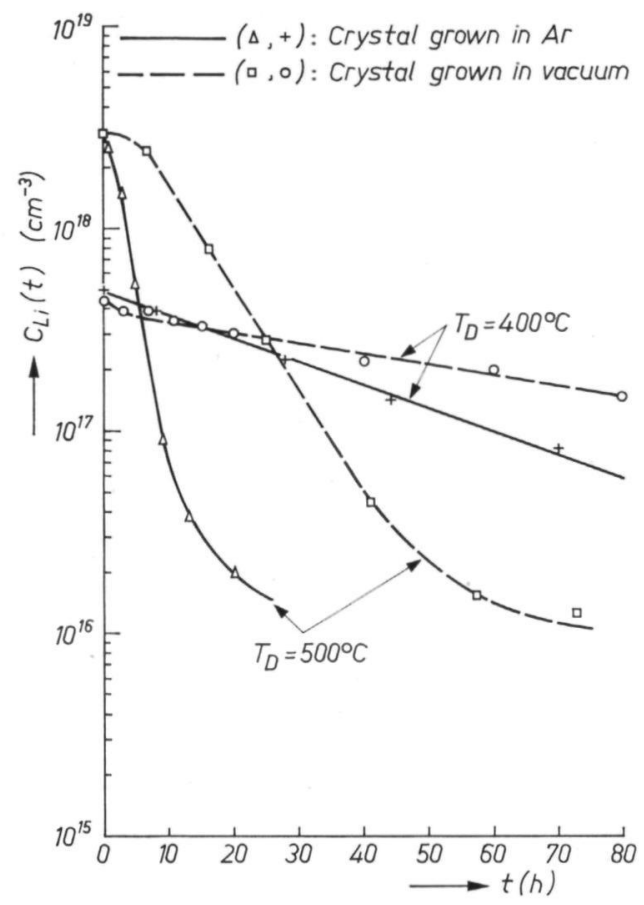

Fig. 5.10. Lithium-precipitation curves for dislocation-free crystals grown in argon and vacuum. $T_{D}=400,500^{\circ} \mathrm{C}$; annealing temperature $160{ }^{\circ} \mathrm{C}$.

\section{TABLE 5-II}

The relation between the diffusion temperature of lithium and the calculated $N_{\mathrm{p}}$-values for crystals grown in argon or vacuum

\begin{tabular}{c|c|c|c}
\hline atmosphere & $V_{g}(\mathrm{~mm} / \mathrm{min})$ & \multicolumn{2}{|c}{$N_{\mathrm{p}}\left(\mathrm{cm}^{-3}\right)$} \\
\cline { 3 - 4 } & & $\begin{array}{c}T_{D}=400^{\circ} \mathrm{C} \\
(\text { diffusion time: } \\
\left.4 \cdot 5-5 \mathrm{~h}){ }^{*}\right)\end{array}$ & $\begin{array}{c}T_{D}=500^{\circ} \mathrm{C} \\
\text { (diffusion time: } \\
6 \mathrm{~h})\end{array}$ \\
\hline argon & 3 & $8 \cdot 4 \cdot 10^{7}$ & $2 \cdot 5 \cdot 10^{9}$ \\
vacuum & $1 \cdot 8$ & $2 \cdot 1.10^{7}$ & $10^{8}$ \\
\hline
\end{tabular}

*) See sec. 4.4 . 
centres are not formed. This is consistent with the results reported by Corbett et al. ${ }^{5-14}$ ), who found that the $\mathrm{VO}_{3}$ complexes are formed at annealing temperatures between 450 and $600{ }^{\circ} \mathrm{C}$.

In the hydrogen-doped crystals, however, either with or without clusters, $\mathrm{VO}_{3}$ complexes are not formed at $500{ }^{\circ} \mathrm{C}$ (figs $5.6(b), 5.7$ and 5.8). This strongly indicates that hydrogen in the silicon lattice indeed reduces or even eliminates the association of vacancies and oxygen atoms.

\subsubsection{The incubation time for cluster formation}

In fig. 5.11 the concentration of A- and B-clusters is plotted versus growth rate for growth in an $\mathrm{Ar}+10 \% \mathrm{H}_{2}$ atmosphere. If hydrogen only reduces the diffusion-limited generation rate of $\mathrm{V}_{m} \mathrm{O}_{n}$ associates, one would expect a decrease in cluster concentration, but not a complete elimination of these defects.

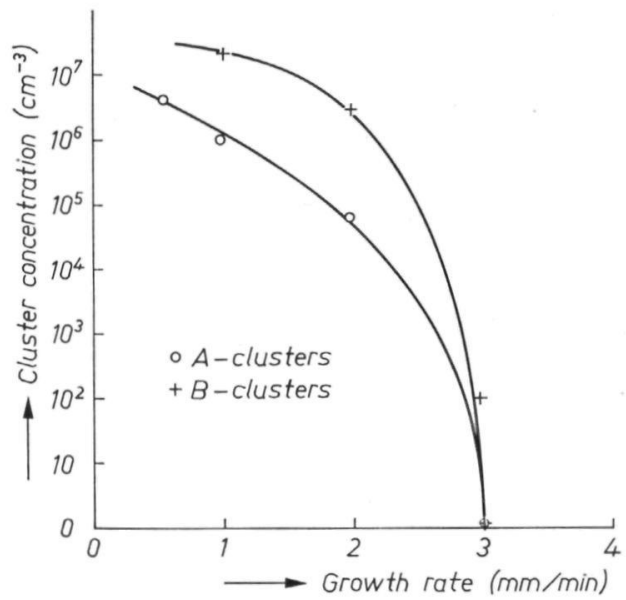

Fig. 5.11. The concentration of A- and B-clusters versus growth rate. Atmosphere: argon $+10 \%$ hydrogen.

As discussed, however, in sec. 4.7, the formation of $\mathrm{V}_{m} \mathrm{O}_{n}$ associates will exhibit incubation times. Because hydrogen doping lowers the oxygen mobility, these incubation times will be increased. The complete elimination of A-cluster formation at $V_{g}=3 \mathrm{~mm} / \mathrm{min}$ indicates that the critical temperature interval $T_{c}-T_{f}$ is passed within this new incubation time for $\mathrm{V}_{m} \mathrm{O}_{n}$-complex formation $\left(t_{0}\right)$. Under these conditions the cooling rate of the crystal amounts to about $2{ }^{\circ} \mathrm{C} / \mathrm{s}$. Consequently we have: $t_{0} \geqslant \frac{1}{2}\left(T_{c}-T_{f}\right) \mathrm{s}$.

\subsubsection{The perfection of vacancy-cluster-free crystals}

Dislocation-free silicon crystals, in which the vacancy-cluster formation has been eliminated according to one of the methods outlined in this study, will still not be completely perfect. As discussed in sec. 5.3.2, crystals in which the 
vacancy-cluster formation is suppressed by hydrogen addition contain tiny $H$ precipitates These defects, however, can easily be removed by an annealing treatment at $T \geqslant 500{ }^{\circ} \mathrm{C}$ without leaving any detectable lattice distortion behınd

Secondly, the amount of vacancies diffusing out to the crystal surface during growth is small (sec 4 l) Consequently, the elimination of vacancy-cluster formation implies that most of the vacancies are left behind in the lattice after coolıng to room temperature A number of these monovacancies will associate, forming divacancies which are immobile at room temperature (sec 1333 ) Furthermore, many vacancies will associate with impurities such as interstitial oxygen, substitutional phosphorus, etc, forming complexes such as the Acentre, E-centre, etc ( $\sec 1333$ ) These complexes are also immobile at room temperature The remainıng monovacancies will contınue to diffuse out to the crystal surface

\section{REFERENCES}

5-1) P Penning, Phılips Res Repts 13, 79, 1958

5-2) A H Boonstra, Philips Res Repts Suppl 1968, No 3

5-3) Y Yukimoto, A Hırano and Y Sugıoka, Jap J appl Phys 6, 420, 1967

5-4) Y Yukımoto, Jap J appl Phys 7, 348, 1968

5 5) A van Wieringen and $N$ Wdrmoltz, Physica 22, 849, 1956

5-6) J H Crawford Jr, H C Schweinler and D K Stevens, J appl Phys 27, 839, 1956

5-7) H Reiss, Phys Rev 100, 1806, 1955

5-9) W Kaiser and P $H$ Keck, J dppl Phys 28, 882, 1957

5 9) M Yoshimatsu, J phys Soc Japan 18, 335, 1963, Suppl II

5-10) Y Sugita, Jap J appl Phys 4, 962, 1965

5-11) A J R de Kock, F M Beeftınk and K J Schell, Appl Phys Letters 20, 81, 1972

5 12) J W Ferman and R Swalın, in De Boer (ed), Reactivity of solıds, Elsevier Publ Comp, Inc, Houston, 1962, p 264

3-13) J W Ferman, J appl Phys 39, 3771, 1968

5-14) J W Corbett, G D Watkins and R S McDonald, Phys Rev 135, A1381, 1964 


\section{List of symbols}

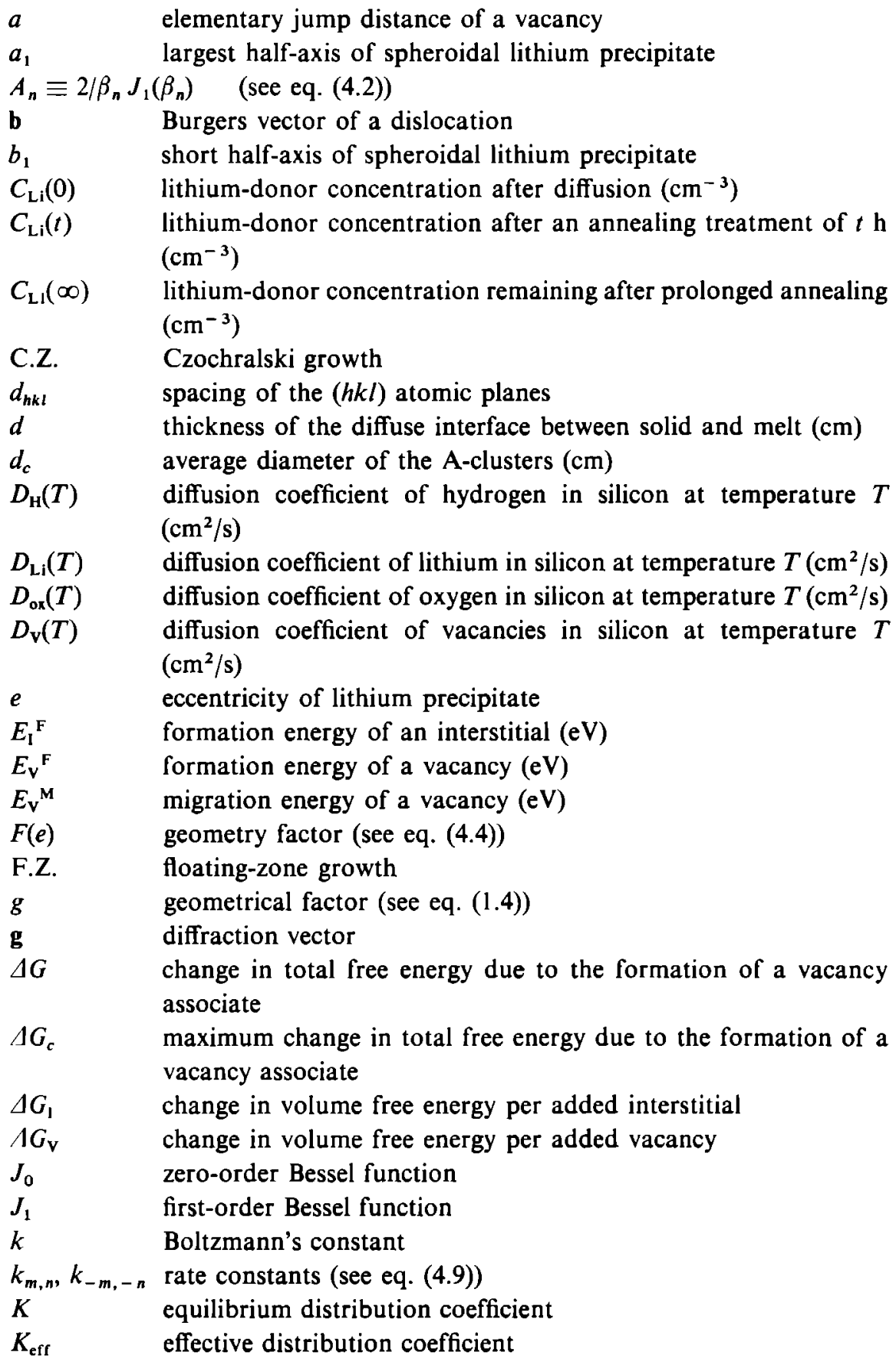


$N_{\mathrm{L}}$

$N_{\text {ox }}$

$N_{\mathrm{p}}$

$N_{\mathrm{s}}$

$N_{\mathrm{v}}(T)$

$N_{\mathrm{v}} \mathrm{s}$

$\Delta N_{\mathrm{V}}$

$N_{\mathbf{V}_{m} \mathbf{o}_{n}}$

R

$R^{\prime}$

$r$

$r_{c}$

$\Delta S_{\mathrm{I}}$

$\Delta S_{\mathrm{V}}$

$t$

$t^{\prime}$

$t_{0}$

$T$

$T_{c}$

$T_{c^{\prime}}$

$T_{\mathrm{D}}$

$T_{\mathrm{f}}$

$T_{M}$

$v$

$V_{g}$

$V_{\mathrm{T}}$

$\beta_{n}$

$\delta$

$\theta_{\mathrm{B}}$

$\Delta \theta$

number of atomic sites in the lattice per $\mathrm{cm}^{3}$

number of interstitial sites in the lattice per $\mathrm{cm}^{3}$

concentration of A-clusters $\left(\mathrm{cm}^{-3}\right)$

concentration of B-clusters $\left(\mathrm{cm}^{-3}\right)$

equilibrium concentration of homogeneously formed nuclei $\left(\mathrm{cm}^{-3}\right)$

equilibrium concentration of interstitials at temperature $T\left(\mathrm{~cm}^{-3}\right)$ concentration of an impurity in the melt $\left(\mathrm{cm}^{-3}\right)$

oxygen concentration in the crystal $\left(\mathrm{cm}^{-3}\right)$

concentration of lithium nucleation sites $\left(\mathrm{cm}^{-3}\right)$

concentration of an impurity in the solid $\left(\mathrm{cm}^{-3}\right)$

equilibrium concentration of vacancies at temperature $T\left(\mathrm{~cm}^{-3}\right)$ concentration of vacancies in the outermost layer of the diffuse solid-liquid interface $\left(\mathrm{cm}^{-3}\right)$

concentration of trapped excess vacancies $\left(\mathrm{cm}^{-3}\right)$

concentration of $\mathrm{V}_{m} \mathrm{O}_{n}$ complexes $\left(\mathrm{cm}^{-3}\right)$

radius of the silicon crystal $(\mathrm{cm})$

crystal-rotation rate $\left(\mathrm{min}^{-1}\right)$

radius $(\mathrm{cm})$

radius of critical nucleus $(\mathrm{cm})$

entropy of formation of an interstitial

entropy of formation of a vacancy

time (s, min, h)

sample thickness

incubation time for vacancy-oxygen-complex formation

absolute temperature (K)

critical temperature below which vacancy condensation on $\mathrm{V}_{m} \mathrm{O}_{n}$ complexes starts

critical temperature below which vacancy condensation on $\mathrm{V}_{m}, \mathrm{O}_{n^{\prime}}$ complexes starts

diffusion temperature for lithium

temperature below which the generation rate of $\mathrm{V}_{m} \mathrm{O}_{n}$ complexes becomes zero

melting point of silicon $\left(1420^{\circ} \mathrm{C}\right)$

precipitate volume per solute atom $\left(\mathrm{cm}^{3}\right)$

crystal-growth rate $(\mathrm{mm} / \mathrm{min})$

target voltage

roots of the equations $J_{0}\left(\beta_{n}\right)=0$

thickness of the diffusion layer in the melt adjacent to the solid-liquid interface $(\mathrm{cm})$

Bragg angle for diffraction

difference in Bragg angle between $K \alpha_{1}$ and $K \alpha_{2}$ radiation 
$\lambda$

$\mu$

$\boldsymbol{\nu}$

$\boldsymbol{\sigma}$

$\tau$

$\Phi$

wavelength

absorption coefficient of X-rays $\left(\mathrm{cm}^{-1}\right)$

apparent jump frequency of a vacancy $\left(\mathrm{s}^{-1}\right)$

surface tension (dyn/cm)

relaxation time for lithium precipitation (s)

horizontal divergence of the X-ray beam used in Lang topography 


\section{Summary}

Silicon single crystals grown from the melt are among the most perfect crystals available, especially since it is possible to produce dislocation-free crystals. Unfortunately, such crystals become supersaturated with vacancies during growth because the equilibrium concentration of these point defects decreases exponentially with decreasing temperature. In the absence of dislocations, which otherwise act as internal vacancy sinks, the excess vacancies are eliminated by the formation of vacancy clusters. The present thesis is devoted to the study of this new class of microdefects.

In chapter 1 the most commonly used methods for growing silicon crystals are described. Attention is also paid to the technique of dislocation elimination, as well as to the concentration and distribution of impurities in these crystals. Finally, some properties of vacancies in the silicon lattice are discussed.

Chapter 2 discusses the various methods of detecting microdefects in silicon which were used in this study. The main methods are preferential etching, copper and lithium decoration, X-ray transmission topography and infrared microscopy.

The crystal perfection of a variety of pure dislocation-free silicon crystals was investigated. The results, given in chapter 3 , show that vacancy clusters are present in a striated distribution. Two types of clusters, differing in size and concentration, can be distinguished. The behaviour of the vacancy clusters during annealing treatments at high temperatures under various conditions is studied as well as the influence of the defects on the perfection of epitaxially grown layers.

The influence of vacancy clusters on the properties of planar diodes is studied by means of the silicon-vidicon device. It is established that the microdefects cause a large increase of the leakage current of reverse-biased diodes.

In chapter 4 the probability of homogeneous and heterogeneous nucleation of vacancy clusters in silicon is discussed and it is concluded that heterogeneous nucleation is dominant. A nucleation model is presented which is based on the assumption that during cooling of the as-grown crystal various types of stable vacancy-oxygen complexes are formed in a lattice supersaturated with vacancies. Some of the vacancy-oxygen complexes are sufficiently large to act as nuclei for the elimination of excess vacancies. At the end of chapter 4 a consistent interpretation based on the model is given of all the experimental observations.

Several methods for elimination of the formation of vacancy clusters during growth can be deduced from the model. These methods are treated in chapter 5 . Completely cluster-free crystals can be grown by one of these methods. 


\section{Samenvatting}

Silicium eenkristallen, gegroeid uit de smelt, behoren tot de meest perfecte materialen welke heden ten dage beschikbaar zijn. Dit geldt in het bijzonder sinds deze kristallen in dislocatievrije vorm gemaakt kunnen worden. Echter, gedurende de groei van dergelijke kristallen treedt er vacature oververzadiging op daar de evenwichtsconcentratie van deze puntfouten exponentieel daalt met dalende temperatuur. $\mathrm{Bij}$ afwezigheid van dislocaties, welke anders als vacature verdwijnplaats fungeren, wordt de overmaat aan vacatures geëlimineerd via de vorming van vacature clusters. Dit proefschrift is gewijd aan de bestudering van deze nieuwe categorie van micro-kristalfouten.

In hoofdstuk 1 worden de meest gangbare kristalgroeimethoden voor silicium behandeld. Tevens wordt hierin aandacht besteed aan de wijze van dislocatie eliminatie. Verder wordt de zuiverheid van silicium kristallen besproken alsmede een aantal eigenschappen van vacatures in het silicium kristalrooster.

De verschillende methodieken, gebruikt in dit onderzoek voor de detectie van micro-kristalfouten worden beschreven in hoofdstuk 2. De meest essentiële methodieken zijn: preferentieel etsen, koper- en lithium-dekoratie, röntgen transmissie topografie en infra-rood microscopie.

De kristalperfektie van een groot aantal zuivere, dislocatievrije silicium kristallen is onderzocht. De resultaten, welke in hoofdstuk 3 worden besproken, tonen de aanwezigheid aan van vacature clusters in een gestrieerd patroon. Twee clustertypen, welke verschillen zowel in grootte als in concentratie, konden worden onderscheiden. Het gedrag van de vacature clusters tijdens temperbehandelingen op hoge temperatuur werd bestudeerd alsmede de invloed van deze micro-defekten op de perfektie van epitaxiaal gegroeide silicium lagen.

De invloed van vacature clusters op de eigenschappen van planaire diodes werd onderzocht met behulp van het silicium-vidicon. Een verhoogde diode lekstroom werd waargenomen ter plaatse van de micro-defekten.

In hoofdstuk 4 wordt het nucleatie proces van de vacature clusters behandeld. Aan de hand van een aantal theoretische beschouwingen alsmede van experimentele gegevens wordt geconcludeerd dat heterogene nucleatie dominant is. Hierna wordt een nucleatie model ontwikkeld, welke is gebaseerd op de aanname dat gedurende het af koelen van het kristal zich stabiele vacature-zuurstof complexen vormen in een kristalrooster dat oververzadigd is met vacatures. Een aantal van deze complexen is voldoende groot om als nucleatiecentra voor de overmaat vacatures te fungeren. Aan het eind van hoofdstuk 4 worden de experimentele waarnemingen verklaard met behulp van het ontwikkelde nucleatie model.

Tevens bleek het mogelijk richtlijnen te geven, gebaseerd op het model, om 
te komen tot een eliminatie van de clustervorming tijdens de kristalgroei. De op deze richtlijnen gebaseerde groeimethoden worden beschreven in hoofdstuk 5. Met behulp van een van deze methoden konden kristallen worden gegroeid waarin de vorming van vacature clusters volledig werd geëlimineerd. 


\section{Curriculum vitae}

De schrijver van dit proefschrift is in 1939 te Amersfoort geboren. In 1957 behaalde hij het einddiploma van de H.B.S.-B van het Christelijk Lyceum te Zeist. Hierna volgde hij de studie in de technische natuurkunde aan de T.H. te Delft, alwaar hij het ingenieursexamen aflegde op 20 oktober 1964. In de periode 1964-1966 vervulde hij zijn militaire dienst bij de Kon. Marine, waar hij belast werd met het geven van onderwijs op het K.I.M. te Den Helder. In juni 1966 volgde in dienst treding op het Natuurkundig Laboratorium van de N.V. Philips' Gloeilampenfabrieken te Eindhoven. 

STELLINGEN

bij het proefschrift van
A. J. R. de Kock

22 maart 1973

Universiteit, Nijmegen 
De vorming van vacature clusters in dislocatie-vrije Si kristallen tijdens de groei uit de smelt kan worden onderdrukt door toepassing van hoge groeisnelheden in combinatie met waterstofdotering.

Hoo「dstuk 5 van dit proefschrift.

II

De verklaring welke Mil'vidskii e.a. geven voor het spontaan verdwijnen van dislocaties tijdens de groei uit de smelt van zwaar gedoteerde halfgeleider kristallen is strijdig met de bestaande experimentele gegevens.

M. G. Mil'vidskii, O. G. Stolyarov en A. V. Berkova, Sov. Physics - Solıd State 6, 2606 (1965).

III

In hun studie betreffende het gedrag van beryllium acceptoren in het Si kristalrooster concluderen Taft en Carlson ten onrechte dat er geen interactie optreedt tussen deze acceptoren en zuurstofatomen.

E. A. Taft en R. O. Carlson, J. Electrochem. Soc. 117, 711 (1970).

\section{IV}

Ten onrechte schrijft Waymouth de experimenteel waargenomen afname in het rendement van een $\mathrm{Hg}$ - $\mathrm{Ar}$ ontlading bij hogere $\mathrm{Hg}$-concentraties toe aan de toename van de effectieve stralingslevensduur van het $\mathrm{Hg}\left(6^{3} P_{1}\right)$-niveau.

J. F. Waymouth, Electric Discharge Lamps, (MIT-Press) 1971, p. 23.

\section{V}

De incorrecte wijze waarop Tung de door hem gemeten epitaxiale groeisnelheid van Si weergeeft als functie van de substraat oriëntatie, voert hem tot de onjuiste conclusie dat deze groeisnelheid maximaal is op (110) oppervlakken.

S. K. Tung, J. Electrochem. Soc. 112, 436 (1965).

VI

De door Burmeister geschatte waarde voor de oppervlakte energie van Si bij hoge temperaturen is waarschijnlijk een factor 2 te laag.

J. Burmeister, J. Crystal Growth 11, 131 (1971). 
De door Solomon e.a. gegeven verklaring voor de permanente efficiency toename van rood-luminescerende GaP-diodes, welke is waargenomen na het kortstondig aanleggen van een hoge voorwaartsspanning, is aanvechtbaar.

R. Solomon en D. DeFevere, App. Phys. Letters 21, 257 (1972).

\section{VIII}

In de analyse, welke Patel e.a. geven van de door hen gemeten vermindering van de anomale transmissie van röntgenstraling in dislocatie-vrij Si ten gevolge van temperbehandelingen, wordt ten onrechte de invloed van vacatures buiten beschouwing gelaten.

J. R. Patel en B. W. Batterman, J. Appl. Phys. 34, 2716 (1963).

IX

Het door Suzuki e.a. waargenomen verdwijnen van stapelfouten in epitaxiale Si lagen bij $500{ }^{\circ} \mathrm{C}$ kan ook worden verklaard met behulp van een vacature transport proces.

T. Suzuki, M. Ura en T. Ogawa, Jap. J. Appl. Phys. 11, 666 (1972).

\section{$\mathrm{X}$}

Het verdient aanbeveling om op gemeentelijk politiek niveau een referendum systeem in te voeren.

\section{XI}

De verkeersveiligheid binnen de bebouwde kom kan worden verhoogd door van alle gelijkvloerse kruisingen voorrangskruisingen te maken. 


